\title{
Cochrane
}

Library

Cochrane Database of Systematic Reviews

\section{Point-of-care ultrasonography for diagnosing thoracoabdominal injuries in patients with blunt trauma (Review)}

Stengel D, Leisterer J, Ferrada P, Ekkernkamp A, Mutze S, Hoenning A

Stengel D, Leisterer J, Ferrada P, Ekkernkamp A, Mutze S, Hoenning A.

Point-of-care ultrasonography for diagnosing thoracoabdominal injuries in patients with blunt trauma.

Cochrane Database of Systematic Reviews 2018, Issue 12. Art. No.: CD012669.

DOI: 10.1002/14651858.CD012669.pub2.

www.cochranelibrary.com

Point-of-care ultrasonography for diagnosing thoracoabdominal injuries in patients with blunt trauma (Review) Copyright $\odot 2018$ The Cochrane Collaboration. Published by John Wiley \& Sons, Ltd. 
TABLE OF CONTENTS

HEADER

ABSTRACT

PLAIN LANGUAGE SUMMARY

SUMMARY OF FINDINGS

BACKGROUND

OBJECTIVES

METHODS

Figure 1.

RESULTS

Figure 2.

Figure 3.

Figure 4.

Figure 5.

Figure 6.

Figure 7.

Figure 8.

Figure 9

DISCUSSION

AUTHORS' CONCLUSIONS

ACKNOWLEDGEMENTS

REFERENCES

CHARACTERISTICS OF STUDIES

DATA

Test 1 . Main analysis set.

Test 2. Sensitivity analysis set with lower sensitivity/specificity values in two original studies.

APPENDICES

CONTRIBUTIONS OF AUTHORS

DECLARATIONS OF INTEREST

SOURCES OF SUPPORT

DIFFERENCES BETWEEN PROTOCOL AND REVIEW

INDEX TERMS 


\section{Point-of-care ultrasonography for diagnosing thoracoabdominal injuries in patients with blunt trauma}

Dirk Stengel ${ }^{1}$, Johannes Leisterer ${ }^{2}$, Paula Ferrada ${ }^{3}$, Axel Ekkernkamp ${ }^{4}$, Sven Mutze ${ }^{5}$, Alexander Hoenning ${ }^{1}$

1Centre for Clinical Research, Department of Trauma and Orthopaedic Surgery, Unfallkrankenhaus Berlin, Berlin, Germany. ${ }^{2}$ ChariteUniversitatsmedizin Berlin, Berlin, Germany. ${ }^{3}$ Department of Surgery, Virginia Commonwealth University, Richmond, VA, USA. ${ }^{4}$ Department of Trauma and Reconstructive Surgery, University Hospital, Greifswald, Germany. ${ }^{5}$ Department of Diagnostic and Interventional Radiology, Unfallkrankenhaus Berlin, Berlin, Germany

Contact address: Dirk Stengel, Centre for Clinical Research, Department of Trauma and Orthopaedic Surgery, Unfallkrankenhaus Berlin, Berlin, 12683, Germany. dirk.stengel@berlin.de, dirk.stengel@ukb.de.

Editorial group: Cochrane Injuries Group.

Publication status and date: New, published in Issue 12, 2018.

Citation: Stengel D, Leisterer J, Ferrada P, Ekkernkamp A, Mutze S, Hoenning A. Point-of-care ultrasonography for diagnosing thoracoabdominal injuries in patients with blunt trauma. Cochrane Database of Systematic Reviews 2018, Issue 12. Art. No.: CD012669. DOI: 10.1002/14651858.CD012669.pub2.

Copyright () 2018 The Cochrane Collaboration. Published by John Wiley \& Sons, Ltd.

\section{A B S T R A C T}

\section{Background}

Point-of-care sonography (POCS) has emerged as the screening modality of choice for suspected body trauma in many emergency departments worldwide. Its best known application is FAST (focused abdominal sonography for trauma). The technology is almost ubiquitously available, can be performed during resuscitation, and does not expose patients or staff to radiation. While many authors have stressed the high specificity of POCS, its sensitivity varied markedly across studies. This review aimed to compile the current best evidence about the diagnostic accuracy of POCS imaging protocols in the setting of blunt thoracoabdominal trauma.

\section{Objectives}

To determine the diagnostic accuracy of POCS for detecting and excluding free fluid, organ injuries, vascular lesions, and other injuries (e.g. pneumothorax) compared to a diagnostic reference standard (i.e. computed tomography (CT), magnetic resonance imaging (MRI), thoracoscopy or thoracotomy, laparoscopy or laparotomy, autopsy, or any combination of these) in patients with blunt trauma.

\section{Search methods}

We searched Ovid MEDLINE (1946 to July 2017) and Ovid Embase (1974 to July 2017), as well as PubMed (1947 to July 2017 ), employing a prospectively defined literature and data retrieval strategy. We also screened the Cochrane Library, Google Scholar, and BIOSIS for potentially relevant citations, and scanned the reference lists of full-text papers for articles missed by the electronic search. We performed a top-up search on 6 December 2018, and identified eight new studies which may be incorporated into the first update of this review.

\section{Selection criteria}

We assessed studies for eligibility using predefined inclusion and exclusion criteria. We included either prospective or retrospective diagnostic cohort studies that enrolled patients of any age and gender who sustained any type of blunt injury in a civilian scenario. Eligible studies had to provide sufficient information to construct a $2 \times 2$ table of diagnostic accuracy to allow for calculating sensitivity, specificity, and other indices of diagnostic test accuracy.

\section{Data collection and analysis}

Two review authors independently screened titles, abstracts, and full texts of reports using a prespecified data extraction form. Methodological quality of individual studies was rated by the QUADAS-2 instrument (the revised and updated version of the original 
Quality Assessment of Diagnostic Accuracy Studies list of items). We calculated sensitivity and specificity with 95\% confidence intervals $(\mathrm{Cl})$, tabulated the pairs of sensitivity and specificity with $\mathrm{Cl}$, and depicted these estimates by coupled forest plots using Review Manager 5 (RevMan 5). For pooling summary estimates of sensitivity and specificity, and investigating heterogeneity across studies, we fitted a bivariate model using Stata 14.0 .

\section{Main results}

We included 34 studies with 8635 participants in this review. Summary estimates of sensitivity and specificity were $0.74(95 \% \mathrm{Cl} 0.65$ to 0.81$)$ and 0.96 ( $95 \% \mathrm{Cl} 0.94$ to 0.98 ). Pooled positive and negative likelihood ratios were estimated at $18.5(95 \% \mathrm{Cl} 10.8$ to 40.5$)$ and 0.27 (95\% $\mathrm{Cl} 0.19$ to 0.37 ), respectively. There was substantial heterogeneity across studies, and the reported accuracy of POCS strongly depended on the population and affected body area. In children, pooled sensitivity of POCS was 0.63 ( $95 \% \mathrm{Cl} 0.46$ to 0.77$)$, as compared to 0.78 ( $95 \%$ $\mathrm{Cl} 0.69$ to 0.84$)$ in an adult or mixed population. Associated specificity in children was $0.91(95 \% \mathrm{Cl} 0.81$ to 0.96$)$ and in an adult or mixed population 0.97 (95\% Cl 0.96 to 0.99$)$. For abdominal trauma, POCS had a sensitivity of $0.68(95 \% \mathrm{Cl} 0.59$ to 0.75$)$ and a specificity of 0.95 (95\% Cl 0.92 to 0.97$)$. For chest injuries, sensitivity and specificity were calculated at $0.96(95 \% \mathrm{Cl} 0.88$ to 0.99$)$ and $0.99(95 \% \mathrm{Cl} 0.97$ to 1.00). If we consider the results of all 34 included studies in a virtual population of 1000 patients, based on the observed median prevalence (pretest probability) of thoracoabdominal trauma of $28 \%$, POCS would miss 73 patients with injuries and falsely suggest the presence of injuries in another 29 patients. Furthermore, in a virtual population of 1000 children, based on the observed median prevalence (pretest probability) of thoracoabdominal trauma of $31 \%$, POCS would miss 118 children with injuries and falsely suggest the presence of injuries in another 62 children.

\section{Authors' conclusions}

In patients with suspected blunt thoracoabdominal trauma, positive POCS findings are helpful for guiding treatment decisions. However, with regard to abdominal trauma, a negative POCS exam does not rule out injuries and must be verified by a reference test such as CT. This is of particular importance in paediatric trauma, where the sensitivity of POCS is poor. Based on a small number of studies in a mixed population, POCS may have a higher sensitivity in chest injuries. This warrants larger, confirmatory trials to affirm the accuracy of POCS for diagnosing thoracic trauma.

\section{PLAIN LANGUAGE SUMMARY}

\section{How accurate is bedside ultrasound for the diagnosis of injuries to the abdomen or chest in patients with blunt injuries?}

\section{Background and aims}

People who sustain a road traffic crash or fall from a height are at risk for blunt body trauma (i.e. non-penetrating trauma) and multiple injuries. Medical professionals caring for these patients in hospital need to know if vital organs or vessels are damaged, and whether there is any major bleeding that requires immediate intervention. Point-of-care sonography (POCS), a form of ultrasound, is a non-invasive, radiation-free, portable imaging technique that can be used at the patient's bedside. It is frequently used to help diagnose injuries in the emergency department. We reviewed the best scientific evidence about the accuracy of POCS, that is its ability to identify or exclude injuries correctly, compared to other diagnostic tests. We considered computed tomography, laparotomy, and autopsy to be good comparative tests against which to measure the accuracy of POCS.

\section{Study characteristics}

We searched for studies from the year in which the first paper about using ultrasound to diagnose trauma patients was published until 15 July 2017. We considered 2296 records and included 34 relevant studies that involved 8635 participants in this review. All 34 studies were published between 1992 and 2017, with the number of participants in each study ranging from 51 to 3181 . Ten studies included only children, two studies only adults, and the remaining 22 studies included both children and adults.

\section{Quality of the evidence}

In many studies, important information about the selection of participants and choice of the diagnostic tests against which to compare POCS was not reported. We therefore rated the methodological quality of the available evidence mostly as unclear.

\section{Key results}

Point-of-care sonography had a sensitivity (i.e. the ability to detect a person with the disease) of $74 \%$ and a specificity (i.e. the ability to exclude a person without the disease) of $96 \%$. Sensitivity and specificity varied considerably across studies, which was due in part to variation in study, participant, and injury characteristics. In children, both the sensitivity and specificity of POCS were lower than in an adult or mixed population, meaning that POCS was less able to identify or rule out an injury. Based on our results, we would expect that amongst 1000 patients of a mixed-age population with suspected blunt trauma to the abdomen or chest, POCS would miss 73 patients with injuries, and would falsely suggest the presence of injuries in 29 patients who were unaffected. This result emphasises the need for additional imaging in trauma patients for whom POCS shows no injuries (i.e. a negative result), to check whether they are really injury-free. 
SUMMARY OF FINDINGS

\section{Summary of findings 1 .}

\begin{tabular}{|c|c|c|c|c|c|c|c|c|}
\hline Population & \multicolumn{8}{|c|}{ Patients of any age and gender who sustained any type of blunt injury in a civilian scenario } \\
\hline Setting & \multicolumn{8}{|c|}{ Clinical evaluation at hospitals of any care level } \\
\hline Index test & \multicolumn{8}{|c|}{ Point-of-care sonography (POCS) as the primary imaging tool } \\
\hline $\begin{array}{l}\text { Reference } \\
\text { standard }\end{array}$ & \multicolumn{8}{|c|}{ Computed tomography (CT), magnetic resonance imaging (MRI), laparotomy, laparoscopy, thoracotomy, thoracoscopy, autopsy } \\
\hline Findings & \multicolumn{8}{|c|}{$\begin{array}{l}\text { 1. POCS emerged as an integral part of trauma algorithms, and remains the point-of-care imaging tool of choice for screening for thoracoabdominal bleeding } \\
\text { in most regions of the world. } \\
\text { 2. Determining the diagnostic accuracy of POCS in patients with blunt trauma may provide clinicians with valuable information on the likelihood of chest and } \\
\text { abdominal injuries and may contribute to decision making regarding the performance of subsequent diagnostic tests. }\end{array}$} \\
\hline Limitations & \multicolumn{8}{|c|}{$\begin{array}{l}\text { 1. Methodological quality was hampered by severe under-reporting in the included studies. We assessed risk of bias as unclear in more than half of the studies } \\
\text { for the domains of patient selection and reference standard, and in one-third of the studies for the index test. } \\
\text { 2. There was substantial heterogeneity among the results of the individual studies, which we investigated further by sources of heterogeneity (see Summary } \\
\text { of findings 2). }\end{array}$} \\
\hline \multirow{2}{*}{$\begin{array}{l}\text { No. of par- } \\
\text { ticipants } \\
\text { (studies) }\end{array}$} & \multirow{2}{*}{$\begin{array}{l}\text { Summary } \\
\text { sensitivity } \\
(95 \% \mathrm{Cl})\end{array}$} & \multirow{2}{*}{$\begin{array}{l}\text { Summary } \\
\text { specificity } \\
(95 \% \mathrm{CI})\end{array}$} & \multirow{2}{*}{$\begin{array}{l}\text { Summary } \\
\text { LR+ }(95 \% \\
\text { CI) }\end{array}$} & \multirow{2}{*}{$\begin{array}{l}\text { Summary } \\
\text { LR- }(95 \% \\
\text { CI) }\end{array}$} & \multirow{2}{*}{$\begin{array}{l}\text { Positive } \\
\text { predictive } \\
\text { value }(95 \% \\
\text { CI) }\end{array}$} & \multirow{2}{*}{$\begin{array}{l}\text { Negative } \\
\text { predictive } \\
\text { value }(95 \% \\
\text { CI) }\end{array}$} & \multicolumn{2}{|c|}{ Consequences in a virtual cohort of $1000^{a}$} \\
\hline & & & & & & & Missed injuries & Overtreated \\
\hline 8635 & 0.74 & 0.96 & 18.5 & 0.27 & 0.88 & 0.90 & 73 & 29 \\
\hline (34) & $\begin{array}{l}\text { (0.65 to } \\
0.81)\end{array}$ & $\begin{array}{l}\text { (0.94 to } \\
0.98)\end{array}$ & $\begin{array}{l}\text { (10.8 to } \\
40.5)\end{array}$ & $\begin{array}{l}\text { (0.19 to } \\
0.37)\end{array}$ & $\begin{array}{l}\text { (0.81 to } \\
0.94)\end{array}$ & $\begin{array}{l}(0.87 \text { to } \\
0.93)\end{array}$ & $\begin{array}{l}\text { (If } 280 \text { people suffer an } \\
\text { injury through trauma, } \\
207 \text { will be identified as } \\
\text { injured, and } 73 \text { will be } \\
\text { missed.) }\end{array}$ & $\begin{array}{l}\text { (If } 720 \text { people do not suffer an in- } \\
\text { jury through trauma, } 29 \text { will be } \\
\text { treated as though they had been } \\
\text { injured, i.e. overtreated.) }\end{array}$ \\
\hline \multicolumn{9}{|c|}{ Sensitivity analysis with a children-only cohort } \\
\hline 1384 & 0.62 & 0.91 & 6.9 & 0.42 & 0.76 & 0.84 & 118 & 62 \\
\hline (10) & $\begin{array}{l}(0.47 \text { to } \\
0.75)\end{array}$ & $\begin{array}{l}(0.81 \text { to } \\
0.96)\end{array}$ & (2.5 to 18.8$)$ & $\begin{array}{l}(0.26 \text { to } \\
0.65)\end{array}$ & $\begin{array}{l}(0.53 \text { to } \\
0.89)\end{array}$ & $\begin{array}{l}(0.77 \text { to } \\
0.90)\end{array}$ & $\begin{array}{l}\text { (If } 310 \text { children suffer an } \\
\text { injury through trauma, } \\
192 \text { will be identified as }\end{array}$ & $\begin{array}{l}\text { (If } 690 \text { children do not suffer an } \\
\text { injury through trauma, } 62 \text { will be } \\
\text { treated as though they had been } \\
\text { injured, i.e. overtreated.) }\end{array}$ \\
\hline
\end{tabular}




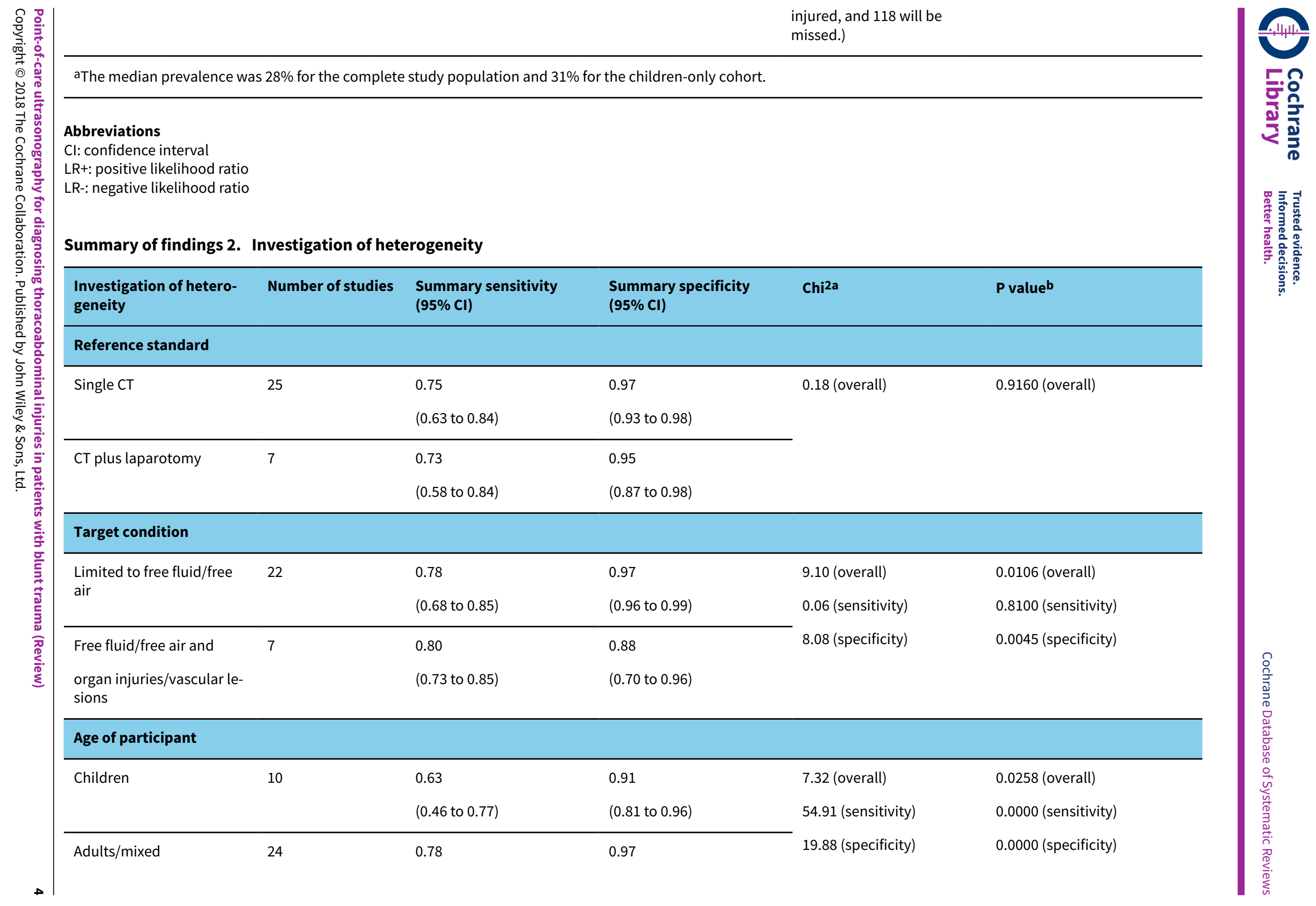




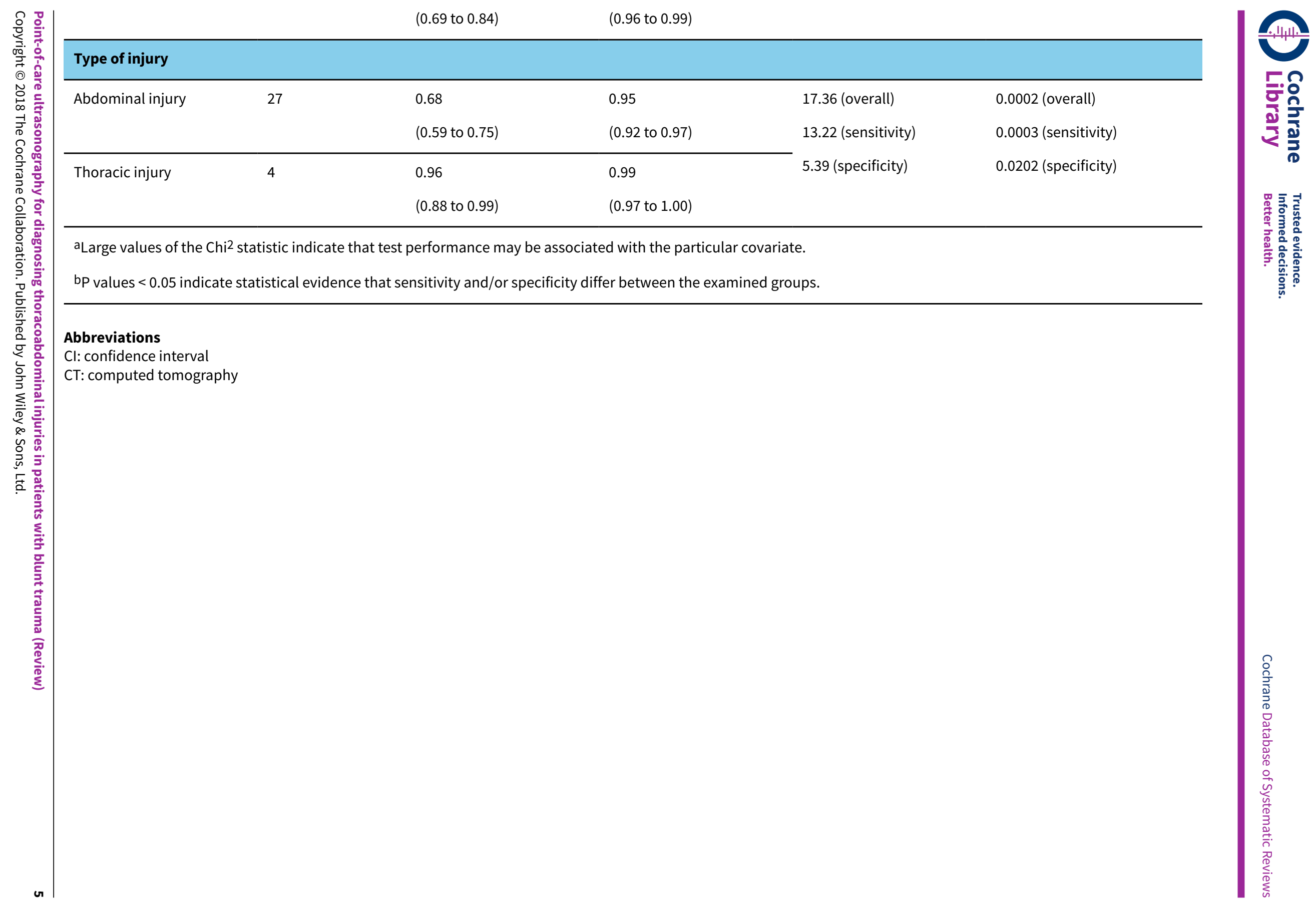




\section{B A C K G R O U N D}

\section{Target condition being diagnosed}

Trauma, including multiple trauma (defined by an Injury Severity Score (ISS) $\geq 16$, or, according to the new Berlin definition, by an Abbreviated Injury Scale (AIS) $\geq$ three for two or more different body regions and one or more additional variables from five physiologic parameters) (Pape 2014), remains a major cause of death and disability worldwide. Severe trauma mainly results from road traffic crashes and falls from a height. In 2010, according to data from the World Health Organization (WHO) Global Burden of Disease Project, motor vehicle crashes ranked eighth in the global death toll (Lozano 2012), and tenth in all sources of disability-adjusted life years (Murray 2012). The WHO and United Nations Decade of Action for Road Safety campaign 2011 to 2020 was launched to raise awareness about this public health concern and to implement simple and effective primary prevention measures.

A 'treat first what kills first' strategy is now in place at most trauma centres across the world, fostered by standardised management algorithms such as Advanced Trauma Life Support (ATLS). Key steps of these algorithms are (Chapleau 2013):

1. maintain airways and establish sufficient oxygenation (i.e. by intubation and tube thoracostomy in case of pneumo- or haematothorax);

2. stop traumatic bleeding (e.g. by tourniquets on extremities, pelvic binders and external fixators, surgical or interventional control of haemorrhage, application of antifibrinolytics such as tranexamic acid, and transfusion of blood products, mainly coagulation factors).

Data from the German Trauma Registry suggest an overall mortality of $10 \%$ from severely injured patients managed within organised trauma networks and at high-volume trauma centres (German Trauma Society 2014). There may be a biological threshold in trauma survivability that cannot be overcome by any of the treatment modalities currently available, and extra translational research efforts are needed to make a difference in future. Apart from unsurvivable brain and upper cervical spine injuries, the leading causes of early death in multiple trauma are chest injuries and abdominal and retroperitoneal haemorrhage (Pfeifer 2016). The presence of free fluid surrounding the liver or spleen, capsular tears, organ contusions or lacerations, and vascular lesions influences early decision making in major trauma.

Stabbing (by sharp tools or weapons such as knives) and shooting are associated with a high chance of organ or vessel injury. The distinct location of wounds may point towards significant trauma to the lungs, heart, mediastinum, liver, spleen, thoracic and/or abdominal aorta. The quality and quantity of injuries sustained in civilian settings and armed conflicts differ in many ways (e.g. by type of weapon, gun, or bullet, wound ballistics, protective armour, austere environment (i.e. where medical care is provided under less than optimal sanitary or hospital-like conditions) and others). Most patients with penetrating trauma need immediate surgical exploration (specifically in case of haemodynamic instability), and preoperative imaging has a rather ancillary role in this situation.

In blunt trauma, however, radiographic imaging is an inevitable part of clinical work-up. Physical examination may reveal indirect signs of internal injury (e.g. contusion marks), but these signs are inconsistent and neither sensitive nor specific. Computed tomography (CT) is regarded as the imaging standard in the emergency department and is currently also the undisputed diagnostic reference test in the trauma scenario. If patients are transferred immediately to the operating theatre before CT imaging, emergency laparotomy, laparoscopy, or thoracotomy is the reference standard of choice. If patients die in the emergency department before any imaging or surgical procedure can be undertaken, definitive diagnoses are obtained during pathological or forensic autopsy. Point-of-care sonography (POCS), however, can be performed during resuscitation, repeated wherever and whenever needed, and does not involve exposure to radiation.

Point-of-care sonography has emerged as an integral part of trauma algorithms and is the initial screening modality of choice for thoracoabdominal bleeding in most regions of the world. Like any other imaging procedure or diagnostic test used for screening purposes, it is important to verify that:

1. a negative index test result is reliable for excluding the condition of interest (guaranteeing that episodes of haemodynamic instability during decompressive brain surgery or fixation of spine, pelvic, or femoral fractures are not caused by sudden major abdominal, thoracic, or retroperitoneal bleeding);

2. a positive index test result is reliable for proving the condition of interest (thus minimising the number of negative or unnecessary thoracotomies and laparotomies, or their minimally invasive equivalents).

Both false-negative and false-positive findings of POCS may misguide trauma teams and affect care priorities adversely.

Diagnostic accuracy (or efficacy) is the first level of the FrybackThornbury hierarchy of evaluating the utility of a diagnostic test procedure (Fryback 1991). While the value and utility of a certain test cannot be derived from its accuracy alone, it would be absurd to ask for the effectiveness or efficiency of an inaccurate diagnostic test.

Determining accuracy is thus the first indispensable step in health technology assessment of POCS. This review aimed to generate the best available evidence about the diagnostic accuracy of clinical ultrasound imaging protocols in the setting of thoracoabdominal and multiple trauma compared to appropriate reference standards. It will guide clinicians regarding the likelihood of chest and abdominal injuries given certain prior probabilities and ultrasound findings, and may facilitate the decision to perform a CT scan or to schedule patients for emergency laparoscopy or laparotomy, or other interventional procedures.

Given the (higher) potential utility and value of POCS in blunt compared to penetrating trauma, this review considered only original studies that included participants with blunt injuries or, in a mixed population, provided sufficient details to explore the accuracy of POCS in this group.

Another aspect that requires scrutiny is the use of POCS in paediatric trauma algorithms. Children are vulnerable to radiation for diagnostic purposes, and their lifetime-attributable risk (LAR) of cancer due to medical imaging must be kept to the necessary minimum. Still, there may be situations in which acute and potentially life-threatening conditions require radiation-emitting (i.e. multi-detector row computed tomography (MDCT)) rather 
than radiation-free imaging techniques (e.g. POCS or magnetic resonance imaging $(\mathrm{MRI}))$.

\section{Index test(s)}

Ultrasound has emerged as a standard for bedside imaging in emergency departments worldwide. Technological progress has led to increasingly lighter and mobile (i.e. handheld) equipment (also available in the preclinical setting, e.g. on helicopters or rescue vehicles). Further advancements include colour-duplex, contrast-enhanced imaging, and even three-dimensional (3D) scanning.

In the trauma setting, POCS is typically performed as focused abdominal sonography for trauma (FAST) (Scalea 1999). In its basic form, FAST includes oblique views of the left upper, right upper, left lower, and right lower abdominal quadrants, as well as a sagittal scan of the mid-abdomen and a transverse view of the pelvic region. The key target of the original FAST scan is free fluid as a surrogate of blood or active bleeding.

The genuine FAST protocol has been modified and supplemented in many ways. The most useful and technically simple extensions were to screen for haematothorax (using oblique or intercostal planes, or both) and, by a xiphoid view, for pericardial effusion. Point-of-care sonography has also proved to be reliable in detecting pneumothorax (Blaivas 2005). Skilled examiners may be able to show and grade abdominal organ injury, although this is likely to exceed the diagnostic limits of POCS in the early resuscitation phase.

In this review, we have used the term POCS rather than FAST because of the varying definitions and targets established in different centres and countries. In clinical practice, ultrasound (or ultrasonography) as an imaging technique is commonly abbreviated and understood as sonography. Altogether, the technological evolution of hardware, increasing skills of operators, and significant advancements in picture acquisition and processing have changed the view of healthcare providers about the role of ultrasonography in the critical care setting substantially. Ultrasound has evolved from a rough screening tool to a conclusive imaging modality.

The index test for this review was therefore any clinical POCS application performed in the setting of blunt trauma that is intended to detect direct or indirect signs of injuries of the thoracic, abdominal, or retroperitoneal cavity or space and/or its organs and vessels.

\section{Clinical pathway}

Clinical examination alone has little - if any - role in excluding injuries to the chest or abdomen. The presence of external injuries, such as seatbelt marks, may increase the likelihood of visceral tears, but their absence does not exclude important trauma. Currently, all major trauma algorithms incorporate thoracoabdominal POCS as a diagnostic imaging tool. However, the interpretation of ultrasound images depends on the experience and clinical background of individual operators. This subjective component influences decision making, and hampers comparisons between initial and follow-up scans taken by different examiners. In 2013, Van Vugt and colleagues published an evidence-based workup protocol for blunt trauma that illustrated the benefits of training trauma teams in POCS (FAST) in combination with an ATLS course (Van Vugt 2013).

\section{Alternative test(s)}

Currently, POCS is challenged by the liberal early use of MDCT, either as abdominal, thoracic, thoracoabdominal, or whole-body MDCT. The latter has emerged as the diagnostic modality of choice in most European trauma centres, and is used in the USA and other high-income nations as well. The so-called 'panscan' usually comprises a native cranial CT, followed by a contrast-enhanced CT from the skull base to the pelvis and/or trochanteric region. Whole-body MDCT is highly specific, thereby minimising false-positive findings (Stengel 2012), and may thus influence care priorities according to the 'treat first what kills first' rule. Data from the German Trauma Registry suggest that the pan-scan improves survival in both unselected trauma cohorts and haemodynamically unstable patients (Huber-Wagner 2013). However, there are concerns regarding excess exposure to radiation caused by uncritical use of the pan-scan at both the individual and population level (Asha 2012). While dose-reducing reconstruction and processing algorithms are available, it is debatable whether they produce images that are similar in quality and diagnostic certainty to those produced by conventional protocols.

The pan-scan is not only a competing imaging tool; it is also regarded as the diagnostic reference standard to which POCS findings must be compared. This leads to an interesting methodological conflict, as it will be almost impossible to compare both imaging modalities in a head-to-head fashion in the trauma scenario.

\section{Rationale}

In high-income countries, it is doubtful whether Pocs findings influence treatment decisions in severe trauma. This can be illustrated by the following four possible scenarios.

1. POCS is positive for free abdominal or thoracic fluid, or both, in a haemodynamically stable patient. This will prompt a CT scan (usually a pan-scan) to identify bleeding sources. In most cases, haemostatic transfusion (plus transarterial embolisation (TAE)) and intensive care unit (ICU) monitoring will be the treatment of choice in this setting.

2. POCS is negative for free abdominal or thoracic fluid, or both, in a haemodynamically stable patient. This will prompt a CT scan (usually a pan-scan) to verify that there are no active bleeding sources that were missed by ultrasound.

3. POCS is negative for free abdominal or thoracic fluid, or both, in a haemodynamically unstable patient. This will almost always prompt a CT scan (usually a pan-scan) to identify bleeding sources and to decide about TAE or emergency surgery, or both.

4. POCS is positive for free abdominal or thoracic fluid, or both, in a haemodynamically unstable patient. Currently, it is unlikely that stability could not be achieved by haemostatic resuscitation and other critical care efforts to make patients pan-scan ready.

Scenario 4 is relevant, but rare, in the Western world. There are very few occasions in which all resuscitation efforts fail and patients are scheduled for emergency thoracotomy or laparotomy, or both, based on POCS findings alone. Still, these situations occur, and clinical practice guidelines must include recommendations on how to cope with them. 
In middle- and low-income countries, however, POCS (in addition to conventional radiographs) may represent the most sophisticated or only non-invasive diagnostic tool available to detect significant traumatic haemorrhage and guide triage. The Sichuan earthquake in 2008, which killed 69,197 people and left 18,222 missing, was a classic example. Focused abdominal sonography for trauma ultrasound proved to be effective, efficient, and possibly lifesaving under these exceptional circumstances (Zhou 2012). Similar observations were made after the earthquake in Haiti in 2010. The earthquake in Nepal in April 2015 (which killed more than 6000 and left 2.8 million people homeless) demonstrated how FAST can play a role in triaging patients effectively outside the context of clinical research.

\section{O B JE C T IVES}

To determine the diagnostic accuracy of POCS for detection and exclusion of:

1. free fluid in the thoracic or abdominal cavities;

2. organ injuries with or without bleeding in the thoracic or abdominal cavities;

3. vascular lesions of the thoracic or abdominal aorta, or other major vessels; and

4. other injuries (e.g. pneumothorax);

compared to the following diagnostic reference standards: computed tomography (CT; 'pan-scan'), magnetic resonance imaging (MRI), thoracotomy, laparotomy, laparoscopy, thoracoscopy, autopsy, or any combination of these.

\section{Secondary objectives}

The secondary objectives of this review were to investigate the influence of individual study and cohort characteristics such as the:

1. reference standard;

2. target condition;

3. patient age (paediatric versus non-paediatric);

4. patient disease status: type of trauma, type of injury, haemodynamic stability, injury severity or probability of survival;

5. environment;

6. operator's expertise and background;

7. hardware;

8. test thresholds;

on both positive and negative POCS scans.

More details are provided in the Investigations of heterogeneity section of the review.

\section{METHODS}

\section{Criteria for considering studies for this review Types of studies}

We included:
1. either prospective or retrospective diagnostic cohort studies that enrolled patients with blunt trauma who:

a. underwent any type of POCS as primary imaging modality to screen for thoracoabdominal injuries; and

b. also underwent predefined imaging or invasive reference tests to verify POCS results;

2. studies that provided $2 \times 2$ tables (or sufficient information to tabulate results) to allow for calculating sensitivity, specificity, and other indices of diagnostic test accuracy.

We excluded:

1. diagnostic case-control studies comparing patients with known case status to healthy controls, as this creates artificial populations and tends to overestimate sensitivity of the index test;

2. case series and case reports;

3. studies with unclear index or reference tests; and

4. studies that did not allow for creating $2 \times 2$ tables.

\section{Participants}

The target population of this review comprised people of any age or gender who sustained any type of blunt trauma in a civilian scenario and were transferred to a hospital of any care level. Also, in order to be eligible participants had to have undergone POCS as the primary imaging tool and to have been followed up either as inpatients or outpatients with different diagnostic modalities to verify whether the condition of interest was present or absent.

Because of clear differences in clinical management, we deliberately excluded people with penetrating injuries, as well as members of armed forces wounded in the battlefield.

\section{Index tests}

Any type of POCS performed in a trauma setting (e.g. FAST ultrasonography of the abdomen or thorax, or both, or any advanced ultrasound protocol) intended to detect:

1. free fluid (as a surrogate of bleeding) in the abdomen, retroperitoneal space, or chest;

2. injuries to solid organs such as the liver or spleen (including attempts to grade their severity);

3. lesions of major vessels; and

4. other injuries (e.g. pneumothorax, as indicated by air in the pleural space).

Variation in POCS technology and application (e.g. specification of ultrasound machines and probes and how up-to-date they were, and handling of inconclusive test results) is addressed in the Assessment of methodological quality section of the review. We planned to examine its potential influence on diagnostic accuracy estimates in the Investigations of heterogeneity section of the review.

\section{Target conditions}

This review focused on blunt thoracoabdominal and multiple trauma, meaning any blunt, non-penetrating force to the abdomen and chest and both solid and hollow viscera, as well as both major vessels. Target conditions considered by this review included: 
1. free fluid in the:
a. thoracic cavity (uni- or bilateral, where specified);
b. abdominal cavity (by abdominal quadrant, where specified);
c. retroperitoneal space;
d. pericardium; or
e. mediastinum;

2. organ injuries, defined as:
a. liver injuries (e.g. capsular tears, haematoma, tissue lacerations);
b. splenic injuries (e.g. capsular tears, haematoma, tissue lacerations);
c. injuries to other solid organs (e.g. pancreas, kidneys);
d. injuries to hollow viscera; or
e. any other organ laceration detected by ultrasonography;

3. vascular lesions, defined as:
a. dissection or rupture of the thoracic or abdominal aorta, or both;

b. rupture of other vessels such as the iliac arteries;

4. other injuries (e.g. pneumothorax, as indicated by air in the pleural space in the thoracic cavity).

We analysed the effect of different types of target conditions as part of our Investigations of heterogeneity. We categorised target conditions into surrogates of blunt trauma (i.e. free fluid and free air, named limited assessment), and both surrogates and direct signs of organ damage (i.e. organ injuries and vascular lesions, named complete assessment).

\section{Reference standards}

In order to be accepted as a diagnostic reference standard, the deliberate use (and the reasoning for its use) of the particular method needed to be specified. To avoid verification bias, all participants were required to undergo an independent imaging or invasive test, regardless of the initial POCS scan.

We classified the following tests as reference standards to confirm the presence or absence of the target condition:

1. any type of CT scan of the major body cavities (i.e. chest, abdomen, pelvis), either selective or performed as a whole-body scan. We planned to stratify results for the use of intravenous or oral contrast agents, or both, and the time interval between POCS and CT;

2. any type of MRI of the major body cavities;

3. laparotomy (by a median or transverse approach), or laparoscopy, either diagnostic or therapeutic;

4. thoracotomy (by median sternotomy or a clamshell approach), or thoracoscopy, either diagnostic or therapeutic;

5. autopsy, either done by pathologists or forensic examiners.

\section{Search methods for identification of studies}

We developed a reproducible search strategy in major online databases based on recommendations of the Cochrane Diagnostic Test Accuracy (DTA) Group and a systematic review performed previously (Stengel 2005). We sought assistance and advice from the Cochrane Injuries Group and its Information Specialist to create a search algorithm with high sensitivity. We also requested access to the Cochrane Injuries Group Specialised Register and searched the Cochrane Library for relevant studies included in published reviews. Furthermore, we used a snowball procedure to identify related articles and articles cited in the reference lists of individual publications, and used Google Scholar as an additional search tool.

\section{Electronic searches}

We searched the following electronic sources.

1. Ovid MEDLINE (1946 to 15 July 2017).

2. PubMed (not MEDLINE) (1947 to 15 July 2017).

3. Ovid Embase (1974 to 15 July 2017).

Search strategies are shown in Appendix 1.

We performed a further search on 6 December 2018; details of the eight potentially relevant studies identified have been added to the Characteristics of studies awaiting classification and Studies awaiting classification sections, and may be incorporated into the review at the next update.

\section{Searching other resources}

A systematic review by Scherer and colleagues showed that results from studies that have not been published in a full-text format are systematically different from fully published results (Scherer 2007). We therefore searched the BIOSIS database for conference abstracts to identify potentially relevant studies that had not yet been published in a journal format (see Appendix 1).

We planned to contact authors of individual studies by email, letter, or phone, if we considered their results to be important but needed further explanation or raw data. We guaranteed that any data exchange complied with the International Conference on Harmonisation Good Clinical Practice (ICH-GCP) principles and rules and regulations of data safety and security.

\section{Data collection and analysis}

We employed standard operating procedures (SOP) for the selection of studies, data extraction, and recording. This included the following principles:

1. screening of titles, abstracts, and full texts of study reports identified by the search strategy by two review authors working independently;

2. use of a data extraction form (including individual study characteristics, individual patient profiles, definition of procedures, etc.);

3. dual assessment and data entry;

4. dual assessment of methodological quality of individual studies;

5. resolution of conflicts by a third review author.

This guarantees transparency and adherence to Cochrane standards and other recommendations (e.g. those issued by the EQUATOR (Enhancing the QUAlity and Transparency Of health Research) group).

\section{Selection of studies}

Two review authors $(\mathrm{AH}, \mathrm{JL})$ independently screened the titles and abstracts of the identified reports, documenting details of selected studies in a predefined electronic spreadsheet and assessing studies for eligibility in terms of the predefined inclusion and exclusion criteria. If it was not possible to make a decision based on title and abstract alone, the full texts of potentially relevant studies 
Figure 1. Study flow diagram for the search conducted on 15 July 2017.

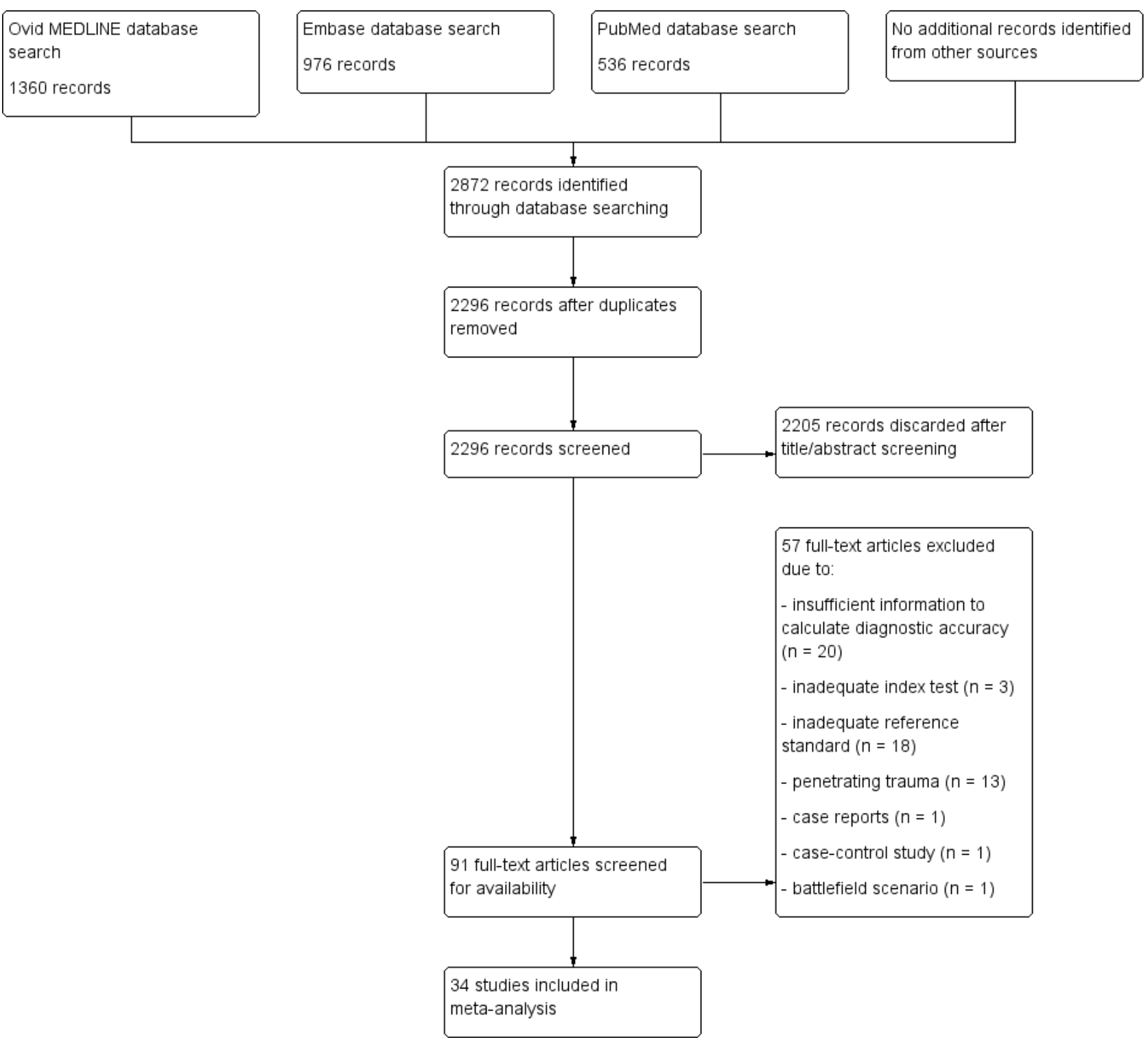

\section{Data extraction and management}

As stated above, we established an SOP for data extraction for systematic reviews, meta-analyses, and health technology assessment (HTA) reports. We adhered to ICH-GCP, Good Epidemiological Practice (GEP), and other relevant rules and recommendations. We have trained personnel on site to record, manage, and audit data, and our data storage modes comply with federal legislation on data safety for research purposes. Two review authors $(\mathrm{AH}, \mathrm{J} \mathrm{L})$ independently extracted data from original papers in duplicate, and resolved discrepancies by discussion, moderated by a third review author (DS). They extracted the following information from published papers:

1. study characteristics (author, year of study, year of publication, journal reference, study design, inclusion/exclusion criteria, operator characteristics, hardware specifications, index test used, reference test used, general setting (urban/rural), mass casualty (yes/no));

2. patient characteristics (age, gender, type of trauma, type of injury, injury severity, haemodynamic stability, probability of survival);

3. outcome of the index test as assessed in the individual studies by diagnosing the target condition and, if available, the number of participants with inconclusive results or who had no test result;

4. diagnostic $2 \times 2$ tables, cross-classifying the disease status on the basis of the reference test (i.e. number of true-positive, falsepositive, false-negative, and true-negative results).

Diagnostic accuracy was expressed by individual and pooled indicators such as sensitivity and specificity with $95 \%$ confidence 
intervals $(\mathrm{Cl})$, positive and negative likelihood ratios (LR), positive predictive value (PPV), negative predictive value (NPV), and the summary receiver operating characteristic curve (SROC).

\section{Assessment of methodological quality}

Two review authors (AH, JL) independently used the QUADAS-2 tool (the revised and updated version of the original Quality Assessment of Diagnostic Accuracy Studies list of items) to assess methodological quality of individual studies (Whiting 2011). Any discrepancies were resolved by discussion, moderated by a third review author (DS). QUADAS-2 includes four main domains, namely: patient selection, index test, reference standard, and flow and timing. We assessed each domain with regard to risk of bias, rating them as 'low', 'high', or 'unclear'. We assessed concerns regarding applicability only for the first three domains, categorising them as 'low', 'high', or 'unclear'. Signalling questions were answered as 'yes', 'no', or 'unclear' (see also Appendix 2). By using tailored review-specific signalling questions we were able to perform a custom-made assessment of the methodological quality of all included studies. We omitted the signalling question "Was any case-control design avoided?" in Domain 1: Patient selection since we did not include any case control study, case series, or case reports. We added three signalling questions to Domain 2: Index test and two signalling questions to Domain 3: Reference test referring to operator's expertise and background, technical features of the hardware, and appropriateness of the ultrasound protocol and reference imaging standard. In Domain 4: Flow and timing, we included the signalling question "Did all participants receive a reference standard?" in order to explore the risk of partial verification bias.

\section{Statistical analysis and data synthesis}

If at least one of the target conditions was detected (i.e. pneumothorax, free fluid, organ or vessel injury), we considered the patient (participant) to be traumatised or test-positive. Otherwise, we considered the participant to be uninjured or test-negative. Our observational unit of interest was thus the individual participant, not a particular injury, and we did not evaluate single target conditions separately in the primary analysis. We used inconclusive test results as reported in the primary studies. For individual studies, we calculated sensitivity and specificity with $95 \% \mathrm{Cl}$, tabulated pairs of sensitivity and specificity with $\mathrm{Cl}$, and depicted estimates by coupled forest plots using Review Manager 5 (RevMan 5) (Review Manager 2014).

Due to the subjective nature of the interpretation of POCS findings, we expected implicit thresholds in test positivity. We assessed a possible threshold effect visually by plotting truepositive rates (sensitivity) from each study against false-positive rates ( 1 - specificity) in a receiver operating characteristic (ROC) space and coupled forest plots of sensitivity and specificity. Since the dichotomous operationalisation of the test result does not enable explicit thresholds, we used the bivariate model according to Reitsma 2005, which is a robust statistical model taking the underlying relationship between sensitivity and specificity into account. The random-effects approach allows for calculating sensitivity and specificity estimates while controlling for heterogeneity across studies. We fitted the models in Stata (Stata 2017) using the 'metandi' command and produced SROC plots using RevMan 5. We estimated average sensitivities and specificities using the bivariate model. We obtained likelihood ratios post estimation using the parameters of the bivariate model (see Summary of findings 1 ).

\section{Investigations of heterogeneity}

We assessed heterogeneity visually by inspecting the coupled forest plots and plots of study results in the SROC space. We also investigated possible sources of heterogeneity by adding single covariates to the basic bivariate random-effects model. We conducted fitting of the bivariate model via the 'xtmelogit' command in Stata (Takwoingi 2016). We investigated the effect of adding covariates by conducting a likelihood ratio test that compared the -2 log likelihoods of the basic bivariate model to a model including a single covariate. If a significant reduction in the -2 log likelihood was detected (indicated by a $P$ value of $<0.05)$, test performance was considered to be associated with the particular covariate. For statistically significant test results, we determined whether the covariate was associated with the estimated sensitivity, specificity, or both (Macaskill 2011), by removing the covariate terms for either sensitivity or specificity, and comparing the fit of each alternative model using likelihood ratio tests.

For tests of heterogeneity, we required a minimum of 10 studies in total and at least four studies per subgroup. We had to dichotomise the covariates we investigated, and differentiate between paediatric and non-paediatric (i.e. adult/ mixed populations), surrogates of injury (e.g. air, free fluid) and organ lacerations, abdominal injuries (i.e. injuries exclusively located in the abdomen) or chest injuries (i.e. injuries exclusively located in the chest), and single CT versus CT plus laparotomy used as reference standard (see Summary of findings 2).

\section{Sensitivity analyses}

We performed sensitivity analyses to investigate how individual QUADAS-2 key domains (i.e. patient selection, index test, reference standard, and flow and timing) affected accuracy estimates, and to explore whether the different evaluations of the two independent review authors within two original studies influenced pooled sensitivities or specificities, or both, of the index test. Moreover, we examined the impact of participants' age on accuracy estimates by only including paediatric studies.

\section{Assessment of reporting bias}

We did not assess reporting bias because there are no accepted ways of doing this for diagnostic test accuracy studies (Deeks 2005).

\section{RE S U L T S}

\section{Results of the search}

We conducted the electronic search on 15 July 2017 in Ovid MEDLINE, PubMed, and Ovid Embase, applying the strategy shown in Appendix 1. We identified 2872 publications including 576 duplicates (Figure 1). After screening the titles or abstracts of 2296 records, 91 studies remained for further evaluation according to our predefined inclusion and exclusion criteria (Types of studies). After screening the full texts of these 91 studies, we discarded 57 and included 34 . We regarded the published data as sufficient to answer our research questions and so did not require individual author contact. 


\section{Included studies}

We extracted information from the 34 included studies according to predefined criteria (Characteristics of included studies). The included studies compared POCS to various imaging and surgical standards (i.e. CT, conventional radiography, laparotomy, thoracotomy, and autopsy) and were published between 1992, Tso 1992, and 2017, Calder 2017, with sample sizes ranging from 51 participants in Benya 2000 to 3181 in Becker 2010. Retrospective and prospective designs were equally distributed, and half of all investigations were conducted in the USA. Ten studies enrolled only children and adolescents, with the age of participants ranging from 1 to 18 years (Benya 2000; Calder 2017; Coley 2000; Corbett 2000; Emery 2001; Fox 2011; Menichini 2015; Soudack 2004; Valentino 2010; Zhou 2012). Two studies included only adults (Blaivas 2005; Verbeek 2014), and 22 studies enrolled participants of any age. Four studies addressed thoracic trauma exclusively (Blaivas 2005; Nandipati 2011; Ojaghi 2014; Zhang 2006). Half of all participants were admitted to level I trauma centres.

\section{Excluded studies}

Fifty-five studies did not meet the inclusion criteria and were excluded (Characteristics of excluded studies). The main reasons for exclusion were insufficient information to allow for calculating diagnostic accuracy $(n=20)$, missing specification of or improper reference standards ( $n=16$, i.e. follow-up ultrasound examination, diagnostic peritoneal lavage (DPL), or clinical observation), or penetrating injuries ( $n=13$ ). Reasons for exclusions are summarised in Figure 1.

\section{Methodological quality of included studies}

We evaluated the methodological quality of individual studies using the QUADAS-2 tool and summarised quality assessments per fulfilled QUADAS-2 domain (Figure 2; Figure 3). Poor reporting, especially in the patient selection and reference standard domains, hampered conclusive judgements about the risk of bias. Only five studies had a low risk of bias in all four critical domains (Benya 2000; Coley 2000; Emery 2001; Soudack 2004; Verbeek 2014). We rated at least two risk-of-bias domains as unclear or high in 19 studies. 
Figure 2. Risk of bias and applicability concerns summary: review authors' judgements about each domain for each included study

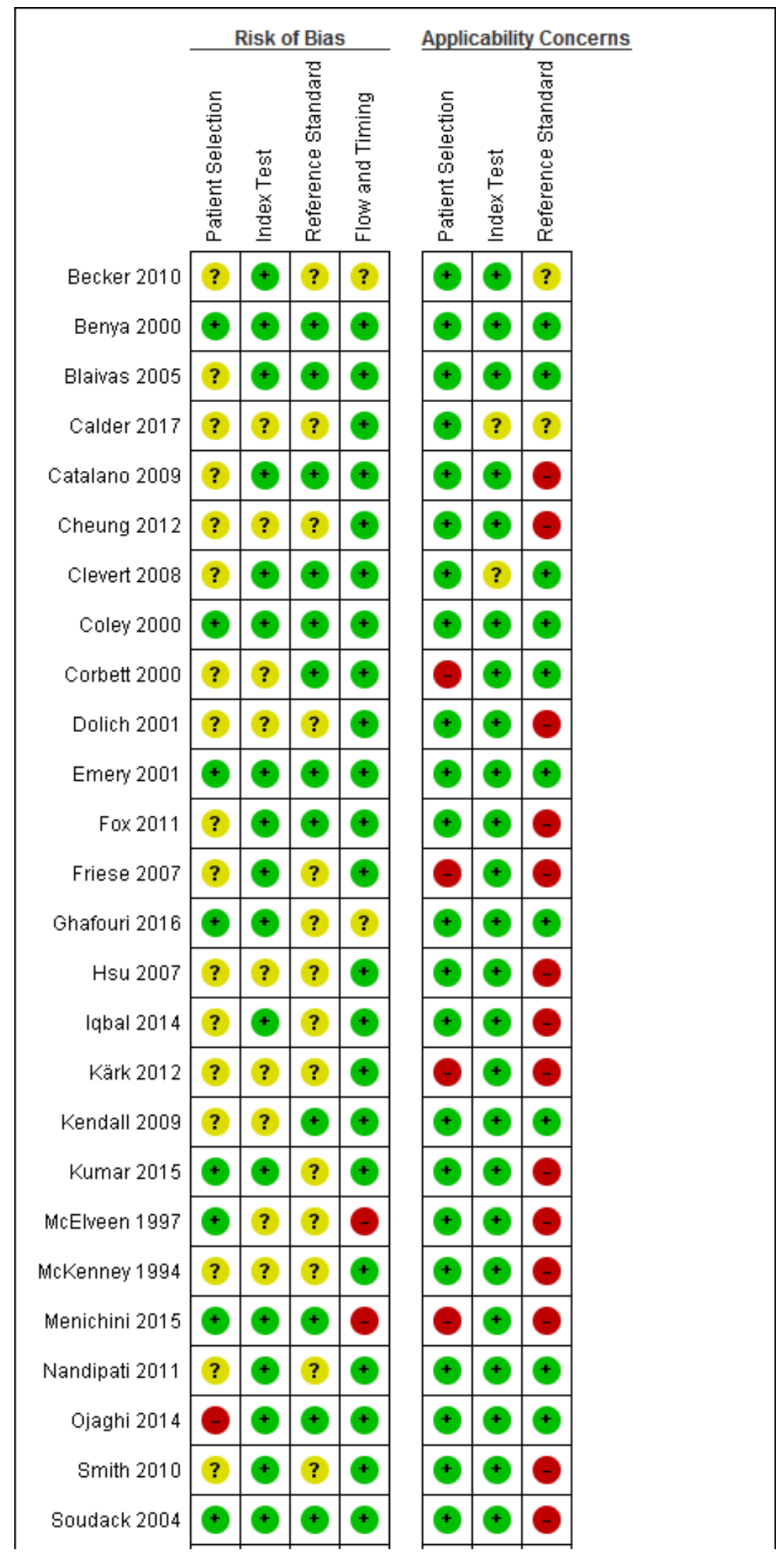

Point-of-care ultrasonography for diagnosing thoracoabdominal injuries in patients with blunt trauma (Review) 
Figure 2. (Continued)

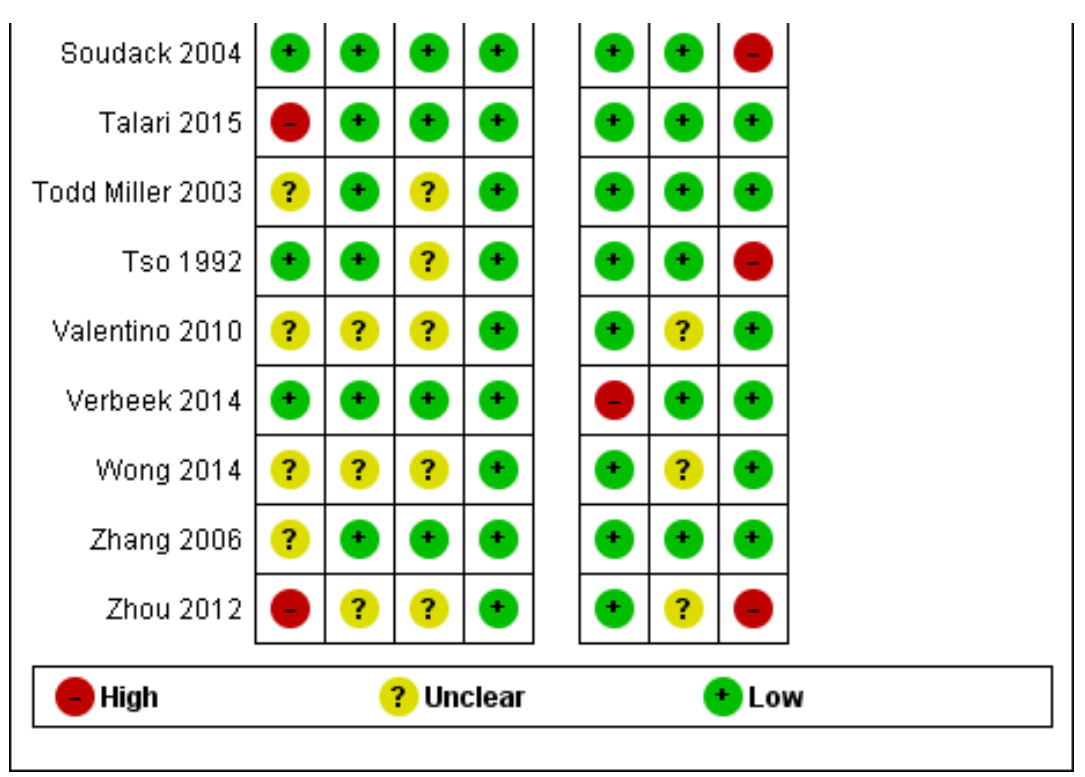

Figure 3. Risk of bias and applicability concerns graph: review authors' judgements about each domain presented as percentages across included studies

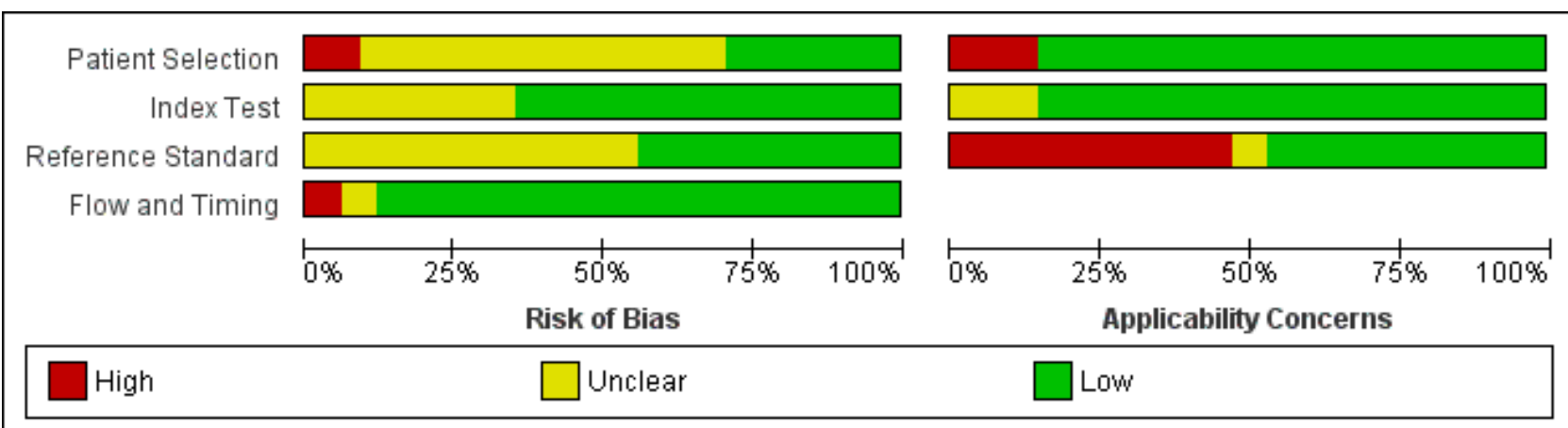

\section{Patient selection}

Three studies had a high risk of bias with regard to patient selection due to non-consecutive enrolment of participants (e.g. inconsistent availability of designated sonographers, refusal of informed consent) and inappropriate exclusions (e.g. exclusion of patients with underlying diseases associated with intra-abdominal fluid) (Ojaghi 2014; Talari 2015; Zhou 2012). Eleven studies had an unclear risk of bias due to non-consecutive enrolment of patients (Becker 2010; Blaivas 2005; Cheung 2012; Clevert 2008; Corbett 2000; Fox 2011; Hsu 2007; Kendall 2009; Smith 2010; Valentino 2010; Wong 2014). We rated the patient inclusion procedure as unclear for 10 studies (Calder 2017; Catalano 2009; Dolich 2001; Friese 2007; Iqbal 2014; Kärk 2012; McKenney 1994; Nandipati 2011; Todd Miller 2003; Zhang 2006).

\section{Index test}

Unclear risk of bias ratings in both index test and reference standard domains originated mainly from missing or unclear information about hardware standards (e.g. machine specifications missing, no information on the number of imaging planes, etc.) or the qualification of operators, or both. Reported qualifications of POCS examiners ranged from attendance of an eight-hour ultrasound course in Hsu 2007 to 10 years of experience in Menichini 2015. We rated eight studies as at unclear risk of bias due to a lack of information regarding the skills of sonographers and insufficient specification about whether index test results were interpreted without knowledge of other imaging test results (Calder 2017; Hsu 2007; Kärk 2012; Kendall 2009; McElveen 1997; McKenney 1994; Wong 2014; Zhou 2012).

\section{Reference standard}

We rated 12 studies as having an unclear risk of bias due to missing technical specifications for the reference imaging test (Calder 2017; Cheung 2012; Dolich 2001; Iqbal 2014; Kärk 2012; Kumar 2015; McElveen 1997; Nandipati 2011; Smith 2010; Tso 1992; Wong 2014; Zhou 2012). Information concerning the diagnostic reference standard was generally far scarcer than details about the index test.

Point-of-care ultrasonography for diagnosing thoracoabdominal injuries in patients with blunt trauma (Review) 


\section{Flow and timing}

Most studies had a low risk of bias with regard to the examination flow and timing domain. Two studies employed diagnostic reference standards conditional on the result of ultrasound exams in some, McElveen 1997, or all, Menichini 2015, of the examined participants.

\section{Findings}

\section{Diagnostic performance of individual studies comparing POCS with reference standard}

Coupled forest plots of individual studies' sensitivities and specificities along with true positives (TP), false positives (FP), false negatives (FN), and true negatives (TN) are depicted in Figure 4. The sensitivity of POCS ranged from $0.26(95 \% \mathrm{Cl} 0.14$ to 0.42$)$ to 1.00 ( $95 \% \mathrm{Cl} 0.74$ to 1.00 ). Specificity ranged from 0.59 (95\% $\mathrm{Cl} 0.44$ to 0.73 ) to $1.00(95 \% \mathrm{Cl} 0.97$ to 1.00$)$. A graphical interpretation of coupled forest plots of individual studies' sensitivities and specificities did not indicate any threshold effect, therefore we considered the bivariate model to be the appropriate pooling procedure.

Figure 4. Coupled forest plots of sensitivity and specificity. TP = true positive; FP = false positive; $F N=$ false negative; $\mathrm{TN}$ = true negative

\begin{tabular}{|c|c|c|c|c|c|c|c|c|c|c|}
\hline Study & TP & FP & FN & TN & Population & Anatomic region & Sensitivity (95\% Cl) & Specificity (95\% Cl) & Sensitivity (95\% Cl) & Specificity (95\% Cl) \\
\hline Wong 2014 & 23 & 16 & 25 & 157 & adultsimixed & abdominal & $0.48[0.33,0.63]$ & $0.91[0.85,0.95]$ & & $\rightarrow$ \\
\hline Verbeek 2014 & 27 & 5 & 15 & 73 & adultsimixed & abdominal & $0.64[0.48,0.78]$ & $0.94[0.86,0.98]$ & 0 & $\rightarrow$ \\
\hline Tso 1992 & 11 & 1 & 5 & 146 & adultsimixed & abdominal & $0.69[0.41,0.89]$ & $0.99[0.96,1.00]$ & & \\
\hline Talari 2015 & 133 & 1 & 27 & 39 & adultsimixed & abdominal & $0.83[0.76,0.89]$ & $0.97[0.87,1.00]$ & & \\
\hline Todd Miller 2003 & 16 & 8 & 22 & 313 & adultsimixed & abdominal & $0.42[0.26,0.59]$ & $0.98[0.95,0.99]$ & & \\
\hline Kendall 2009 & 9 & 3 & 10 & 130 & adultsimixed & abdominal & $0.47[0.24,0.71]$ & $0.98[0.94,1.00]$ & & \\
\hline Kumar 2015 & 37 & 1 & 9 & 3 & adultsimixed & abdominal & $0.80[0.66,0.91]$ & $0.75[0.19,0.99]$ & & \\
\hline lqbal 2014 & 40 & 14 & 12 & 34 & adultsimixed & abdominal & $0.77[0.63,0.87]$ & $0.71[0.56,0.83]$ & & \\
\hline McKenney 1994 & 17 & 0 & 4 & 124 & adultsimixed & abdominal & $0.81[0.58,0.95]$ & $1.00[0.97,1.00]$ & & \\
\hline K\&\#228;rk 2012 & 67 & 0 & 14 & 37 & adultsimixed & abdominal & $0.83[0.73,0.90]$ & $1.00[0.91,1.00]$ & $\rightarrow$ & $\rightarrow$ \\
\hline McElveen 1997 & 9 & 1 & 2 & 58 & adultsimixed & abdominal & $0.82[0.48,0.98]$ & $0.98[0.91,1.00]$ & & $\rightarrow$ \\
\hline Catalano 2009 & 78 & 10 & 21 & 47 & adultsimixed & abdominal & $0.79[0.69,0.86]$ & $0.82[0.70,0.91]$ & - & \\
\hline Cheung 2012 & 20 & 3 & 20 & 110 & adultsimixed & abdominal & $0.50[0.34,0.66]$ & $0.97[0.92,0.99]$ & & \\
\hline Becker 2010 & 352 & 44 & 121 & 2664 & adults/mixed & abdominal & $70,0.78]$ & $0.98[0.98,0.99]$ & n & \\
\hline Friese 2007 & 11 & 2 & 31 & 52 & adultsimixed & abdominal & $.14,0.42]$ & $0.96[0.87,1.00]$ & & $\rightarrow$ \\
\hline Ghafouri 2016 & 27 & 6 & 2 & 85 & adultsimixed & abdominal & $0.93[0.77,0.99]$ & $0.93[0.86,0.98]$ & & $\rightarrow$ \\
\hline Dolich 2001 & 264 & 37 & 43 & 272 & adultsimixed & abdominal & $0.86[0.82,0.90]$ & $0.88[0.84,0.91]$ & & 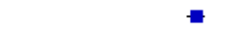 \\
\hline Clevert 2008 & 15 & 0 & 3 & 60 & adultsimixed & abdominal & $0.83[0.59,0.96]$ & $4,1.00]$ & & \\
\hline Smith 2010 & 13 & 0 & 3 & 36 & adultsimixed & other & $0.81[0.54,0.96]$ & $0,1.00]$ & $\rightarrow-$ & $\rightarrow$ \\
\hline Hsu 2007 & 78 & 8 & 22 & 302 & adultsimixed & other & $0.78[0.69,0.86]$ & $0.97[0.95,0.99]$ & $\rightarrow$ & \\
\hline Zhang 2006 & 25 & 3 & 4 & 103 & adultsimixed & thoracic & $0.86[0.68,0.96]$ & $0.97[0.92,0.99]$ & - & $\Rightarrow$ \\
\hline Nandipati 2011 & 12 & 1 & 0 & 146 & adultsimixed & thoracic & $1.00[0.74,1.00]$ & $0.99[0.96,1.00]$ & $\longrightarrow$ & \\
\hline Ojaghi 2014 & 50 & 0 & 2 & 98 & adultsimixed & thoracic & $0.96[0.87,1.00]$ & $1.00[0.96,1.00]$ & $\rightarrow$ & \\
\hline Blaivas 2005 & 52 & 1 & 1 & 122 & adultsimixed & thoracic & $0.98[0.90,1.00]$ & $0.99[0.96,1.00]$ & $\rightarrow$ & \\
\hline Zhou 2012 & 29 & 18 & 6 & 43 & children & abdominal & $0.83[0.66,0.93]$ & $0.70[0.57,0.81]$ & & \\
\hline Soudack 2004 & 34 & 2 & 3 & 70 & children & abdominal & $0.92[0.78,0.98]$ & $0.97[0.90,1.00]$ & & \\
\hline Valentino 2010 & 59 & 20 & 25 & 29 & children & abdominal & $0.70[0.59,0.80]$ & $0.59[0.44,0.73]$ & & \\
\hline Menichini 2015 & 26 & 0 & 39 & 6 & children & abdominal & $0.40[0.28,0.53]$ & $1.00[0.54,1.00]$ & & \\
\hline Calder 2017 & 27 & 21 & 70 & 222 & children & abdominal & $0.28[0.19,0.38]$ & $0.91[0.87,0.95]$ & & $=$ \\
\hline Benya 2000 & 12 & 10 & 5 & 24 & children & abdominal & $0.71[0.44,0.90]$ & $0.71[0.53,0.85]$ & & \\
\hline Emery 2001 & 20 & 14 & 24 & 102 & children & abdominal & $0.45[0.30,0.61]$ & $0.88[0.81,0.93]$ & & $\rightarrow$ \\
\hline Fox 2011 & 12 & 13 & 11 & 321 & children & abdominal & $0.52[0.31,0.73]$ & $0.96[0.93,0.98]$ & & - \\
\hline Coley 2000 & 12 & 2 & 10 & 73 & children & abdominal & $0.55[0.32,0.76]$ & $0.97[0.91,1.00]$ & & $\rightarrow$ \\
\hline Corbett 2000 & 7 & 1 & 2 & 31 & children & other & $0.78[0.40,0.97]$ & $0.97[0.84,1.00]$ & & $\rightarrow$ \\
\hline
\end{tabular}

\section{Estimates derived from the bivariate model comparing POCS with reference standard}

Figure 5 shows the pooled summary point for sensitivity and specificity derived from the bivariate model with corresponding $95 \%$ confidence and prediction regions. Summary estimates of sensitivity and specificity were $0.74(95 \% \mathrm{Cl} 0.65$ to 0.81$)$ and 0.96 (95\% Cl 0.94 to 0.98$)$. Corresponding positive and negative LRs were $18.5(95 \% \mathrm{Cl} 10.8$ to 40.5$)$ and $0.27(95 \% \mathrm{Cl} 0.19$ to 0.37$)$; PPV was 0.88 (95\% Cl 0.81 to 0.94$)$; and NPV was 0.90 (95\% Cl 0.87 to 0.93). The observed median prevalence of blunt thoracoabdominal trauma in the total cohort was $28 \%$. In a virtual population of 1000 patients, assuming the median prevalence of $28 \%$, POCS would miss 73 patients with injuries, and falsely suggest the presence of injuries in another 29 patients. 
Figure 5. Summary receiver operating characteristic (ROC) plot of sensitivity and specificity of all 34 included studies. The solid circle represents the summary estimate of sensitivity and specificity. The summary estimate is surrounded by a dotted line representing the $95 \%$ confidence region and a dashed lined representing the $95 \%$ prediction region.

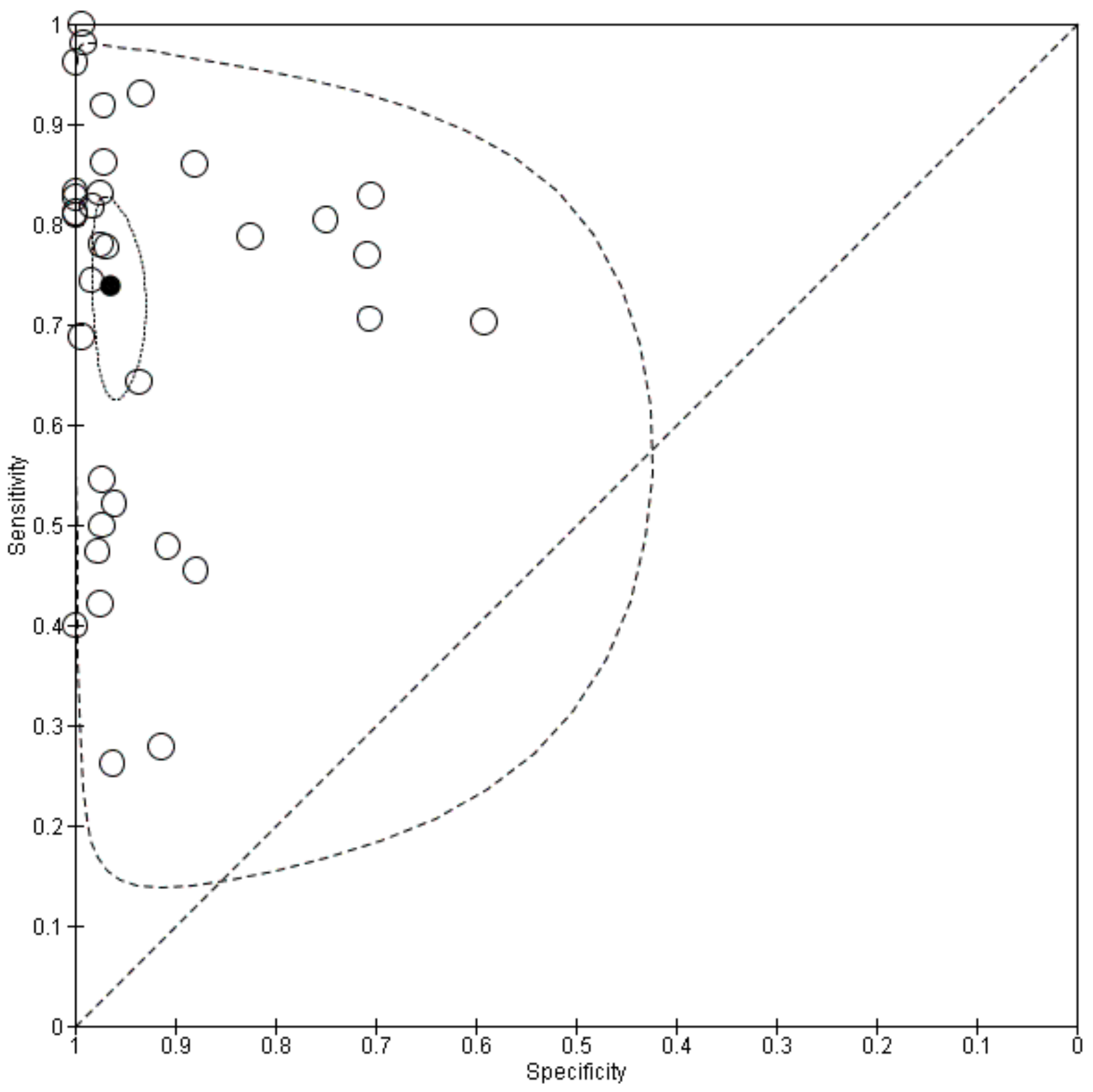

\section{Heterogeneity}

The prediction region around the summary estimate in Figure 5 indicates with $95 \%$ confidence where the true sensitivity and specificity of POCS would be expected in a future study. As indicated by the width of the region, there was considerable heterogeneity between studies. Regarding sensitivity, the 95\% prediction region varied from 0.14 to 0.98 , while the specificity of future studies was estimated to range from 0.42 to 1.00 . This marked between-study heterogeneity needs further exploration.

\section{a. Effect of reference standard}

Each individual study used CT as confirmative imaging modality, either as a single gold standard or in combination with other reference tests. In 25 studies, target conditions were confirmed exclusively with $\mathrm{CT}$, and in seven studies with $\mathrm{CT}$ and laparotomy. There was no difference in POCS sensitivity and specificity when compared with $\mathrm{CT}$ or $\mathrm{CT}$ plus laparotomy $\left(\mathrm{Chi}^{2}=0.18\right.$; $\mathrm{P}$ value (overall effect) $=0.9160 ; \mathrm{CT}: 0.75(95 \% \mathrm{Cl} 0.63$ to 0.84$)$ and $0.97(95 \%$ $\mathrm{Cl} 0.93$ to 0.98 ), CT plus laparotomy: 0.73 (95\% $\mathrm{Cl} 0.58$ to 0.84 ) and 0.95 ( $95 \% \mathrm{Cl} 0.87$ to 0.98$)$ ). 


\section{b. Effect of target condition}

Twenty-two studies assessed the diagnostic accuracy of POCS targeting surrogate measures like free fluid (18 studies) or air (four studies). Three studies aimed to assess solid organ damage, and another seven studies targeted both free fluid and direct signs of organ injuries. The individual target condition mainly affected specificity estimates $\left(\mathrm{Chi}^{2}=9.10 ; \mathrm{P}\right.$ value (overall effect) $=0.0106$ ). Sensitivity of POCS limited to detecting free fluid/air was 0.78 (95\%
$\mathrm{Cl} 0.68$ to 0.85 ), compared to 0.80 (95\% $\mathrm{Cl} 0.73$ to 0.85 ) for complete assessment $\left(\mathrm{Chi}^{2}=0.06 ; \mathrm{P}\right.$ value (pair-wise $\left.)=0.8100\right)$. Related specificities were $0.97(95 \% \mathrm{Cl} 0.96$ to 0.99$)$ and $0.88(95 \% \mathrm{Cl} 0.70$ to 0.96$)$, respectively $\left(\mathrm{Chi}^{2}=8.08\right.$; $\mathrm{P}$ value (pair-wise) $=0.0045$ ) Coupled forest plots for limited assessment (Figure 6) and complete assessment (Figure 7) show greater variation in specificity in studies targeting both free fluid and direct signs of organ injuries compared to studies aimed only at free fluid or free air.

Figure 6. Coupled forest plots of sensitivity and specificity for studies targeting only free fluid or free air $(n=22)$. TP $=$ true positive; $\mathrm{FP}=$ false positive; $\mathrm{FN}$ = false negative; $\mathrm{TN}$ = true negative

\begin{tabular}{|c|c|c|c|c|c|c|c|c|}
\hline Study & TP & FP & FN & TN & Sensitivity (95\% Cl) & Specificity (95\% Cl) & Sensitivity (95\% Cl) & Specificity (95\% Cl) \\
\hline Becker 2010 & 352 & 44 & 121 & 2664 & $0.74[0.70,0.78]$ & $0.98[0.98,0.99]$ & $\Rightarrow$ & \\
\hline Blaivas 2005 & 52 & 1 & 1 & 122 & $0.98[0.90,1.00]$ & $0.99[0.96,1.00]$ & $\rightarrow$ & \\
\hline Cheung 2012 & 20 & 3 & 20 & 110 & $0.50[0.34,0.66]$ & $0.97[0.92,0.99]$ & & \\
\hline Coley 2000 & 12 & 2 & 10 & 73 & $0.55[0.32,0.76]$ & $0.97[0.91,1.00]$ & & \\
\hline Corbett 2000 & 7 & 1 & 2 & 31 & $0.78[0.40,0.97]$ & $0.97[0.84,1.00]$ & & $\rightarrow$ \\
\hline Emery 2001 & 20 & 14 & 24 & 102 & $0.45[0.30,0.61]$ & $0.88[0.81,0.93]$ & & $\rightarrow$ \\
\hline Fox 2011 & 12 & 13 & 11 & 321 & $0.52[0.31,0.73]$ & $0.96[0.93,0.98]$ & & \\
\hline Friese 2007 & 11 & 2 & 31 & 52 & $0.26[0.14,0.42]$ & $0.96[0.87,1.00]$ & 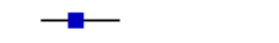 & 7 \\
\hline Ghafouri 2016 & 27 & 6 & 2 & 85 & $0.93[0.77,0.99]$ & $0.93[0.86,0.98]$ & & $\rightarrow$ \\
\hline Hsu 2007 & 78 & 8 & 22 & 302 & $9.86]$ & $0.97[0.95,0.99]$ & - & \\
\hline lqbal 2014 & 40 & 14 & 12 & 34 & 87] & $0.83]$ & - & $\rightarrow-$ \\
\hline Kumar 2015 & 37 & 1 & 9 & 3 & $0.80[0.66,0.91]$ & $0.99]$ & $\rightarrow-$ & \\
\hline K\&\#228;rk 2012 & 67 & 0 & 14 & 37 & $0.83[0.73,0.90]$ & $1.00]$ & $\rightarrow$ & \\
\hline McKenney 1994 & 17 & 0 & 4 & 124 & $0.81[0.58,0.95]$ & $1.00[0.97,1.00]$ & -1 & \\
\hline Nandipati 2011 & 12 & 1 & 0 & 146 & $1.00[0.74,1.00]$ & $0.99[0.96,1.00]$ & $\longrightarrow$ & \\
\hline Ojaghi 2014 & 50 & 0 & 2 & 98 & $0.96[0.87,1.00]$ & $1.00[0.96,1.00]$ & $\rightarrow$ & \\
\hline Smith 2010 & 13 & 0 & 3 & 36 & $0.81[0.54,0.96]$ & $1.00[0.90,1.00]$ & $=$ & \\
\hline Soudack 2004 & 34 & 2 & 3 & 70 & $0.92[0.78,0.98]$ & $0.97[0.90,1.00]$ & $\rightarrow$ & \\
\hline Talari 2015 & 133 & 1 & 27 & 39 & $0.83[0.76,0.89]$ & $0.97[0.87,1.00]$ & 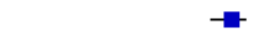 & \\
\hline Todd Miller 2003 & 16 & 8 & 22 & 313 & $0.42[0.26,0.59]$ & $0.98[0.95,0.99]$ & & \\
\hline Verbeek 2014 & 27 & 5 & 15 & 73 & $0.64[0.48,0.78]$ & $0.94[0.86,0.98]$ & & $\rightarrow$ \\
\hline Zhang 2006 & 25 & 3 & 4 & 103 & $0.86[0.68,0.96]$ & $0.97[0.92,0.99]$ & 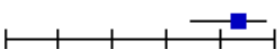 & \\
\hline
\end{tabular}

Figure 7. Coupled forest plots of sensitivity and specificity for studies considering both surrogates and organ lacerations $(n=7)$. TP = true positive; $F P=$ false positive; $F N=$ false negative; $T N=$ true negative

\begin{tabular}{|c|c|c|c|c|c|c|c|c|}
\hline Study & TP & FP & $\mathbf{F N}$ & TN & Sensitivity $(95 \% \mathrm{Cl})$ & Specificity (95\% Cl) & Sensitivity (95\% Cl) & Specificity ( $95 \% \mathrm{Cl})$ \\
\hline Benya 2000 & 12 & 10 & 5 & 24 & $0.71[0.44,0.90]$ & $0.71[0.53,0.85]$ & & $\longrightarrow$ \\
\hline Catalano 2009 & 78 & 10 & 21 & 47 & $0.79[0.69,0.86]$ & $0.82[0.70,0.91]$ & & $\rightarrow-$ \\
\hline Dolich 2001 & 264 & 37 & 43 & 272 & $0.86[0.82,0.90]$ & $0.88[0.84,0.91]$ & & $=$ \\
\hline McElveen 1997 & 9 & 1 & 2 & 58 & $0.82[0.48,0.98]$ & $0.98[0.91,1.00]$ & & \\
\hline Tso 1992 & 11 & 1 & 5 & 146 & $0.69[0.41,0.89]$ & $0.99[0.96,1.00]$ & $\rightarrow$ & \\
\hline Valentino 2010 & 59 & 20 & 25 & 29 & $0.70[0.59,0.80]$ & $0.59[0.44,0.73]$ & $\rightarrow$ & $\rightarrow-$ \\
\hline Zhou 2012 & 29 & 18 & 6 & 43 & $0.83[0.66,0.93]$ & $0.70[0.57,0.81]$ & & $=\frac{-1}{1}$ \\
\hline
\end{tabular}

\section{c. Effect of participant age}

Ten studies included only children under 18 years of age, whereas 24 studies involved adults or a largely adult population. Participant age was associated with significantly different estimates of both sensitivity and specificity $\left(\mathrm{Chi}^{2}=7.32 ; \mathrm{P}\right.$ value (overall effect $)=$ 0.0258). Pooled sensitivity of POCS was 0.63 (95\% $\mathrm{Cl} 0.46$ to 0.77 ) in children and $0.78(95 \% \mathrm{Cl} 0.69$ to 0.84$)$ in an adult or mixed population $\left(\mathrm{Chi}^{2}=54.91 ; \mathrm{P}\right.$ value (pair-wise $\left.)<0.0001\right)$. Associated

specificities were $0.91(95 \% \mathrm{Cl} 0.81$ to 0.96$)$ and $0.97(95 \% \mathrm{Cl} 0.96$ to 0.99$)\left(\mathrm{Chi}^{2}=19.88\right.$; $\mathrm{P}$ value (pair-wise) $\left.<0.0001\right)$. Figure 8 depicts individual sensitivity and specificity estimates along with summary points, 95\% confidence regions, and 95\% prediction regions for both paediatric and non-paediatric studies. Trials including only children are depicted by means of black dots, while trials with a predominantly adult population are shown as red dots. Figure 4 illustrates sensitivities and specificities from individual studies for 
non-paediatric (first 24 studies) and paediatric populations (last 10 studies).

Figure 8. Summary receiver operating characteristic (ROC) plot of sensitivity and specificity: paediatric studies ( $n=10$; indicated in black) versus non-paediatric studies ( $n=24$; indicated in red). The solid circles represent the summary estimates of sensitivity and specificity. The summary estimates are surrounded by a dotted line representing the $95 \%$ confidence region and a dashed lined representing the $95 \%$ prediction region.

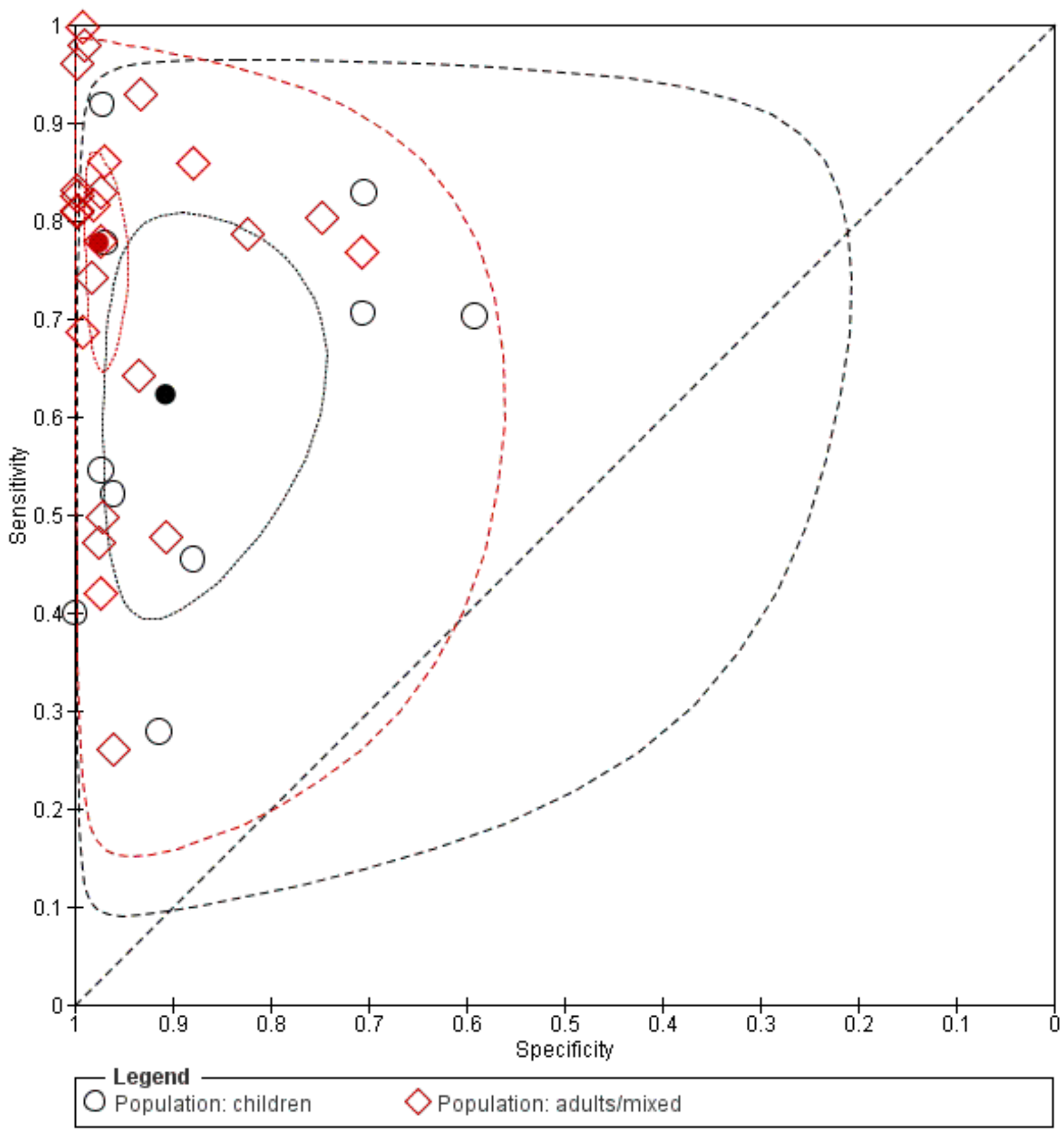

\section{d. Effect of type of injury}

Twenty-seven studies targeted abdominal injuries; four studies addressed thoracic trauma; two studies addressed both; and one study examined blunt truncal trauma without further specification.
We tested the influence of the anatomic region on the basic model by adding a binary covariate that dichotomised the injury type into thoracic or abdominal trauma. Based on 31 studies, this led to significantly different estimates of sensitivity and specificity 
$\left(\mathrm{Chi}^{2}=17.36 ; \mathrm{P}\right.$ value (overall effect $\left.)=0.0002\right)$. Sensitivity of POCS for abdominal and thoracic trauma was $0.68(95 \% \mathrm{Cl} 0.59$ to $0.75)$ and $0.96(95 \% \mathrm{Cl} 0.88$ to 0.99$)$, respectively $\left(\mathrm{Chi}^{2}=13.22\right.$; $\mathrm{P}$ value (pair-wise) $=0.0003)$. Specificity was $0.95(95 \% \mathrm{Cl} 0.92$ to $0.97)$ and $0.99(95 \% \mathrm{Cl} 0.97$ to 1.00$)$, respectively $\left(\mathrm{Chi}^{2}=5.39 ; \mathrm{P}\right.$ value (pair-wise) $=0.0202$ ). Individual sensitivities and specificities for abdominal trauma, thoracic trauma, and trauma that is not exclusively abdominal or thoracic (i.e. both abdominal and thoracic trauma, truncal trauma) are displayed in Figure 4. Individual and average estimates of sensitivity and specificity, and both 95\% confidence regions and $95 \%$ prediction regions around the summary estimates for thoracic and abdominal studies separately are illustrated in Figure 9. For abdominal trauma, the accuracy values are widely scattered across studies, whereas sensitivity and specificity values are consistently high when targeting only thoracic trauma. 
Figure 9. Summary receiver operating characteristic (ROC) plot of sensitivity and specificity: abdominal studies ( $n=27$; indicated in black) versus thoracic studies ( $n=4$; indicated in red). The solid circles represent the summary estimates of sensitivity and specificity. The summary estimates are surrounded by a dotted line representing the $95 \%$ confidence region and a dashed lined representing the $95 \%$ prediction region.

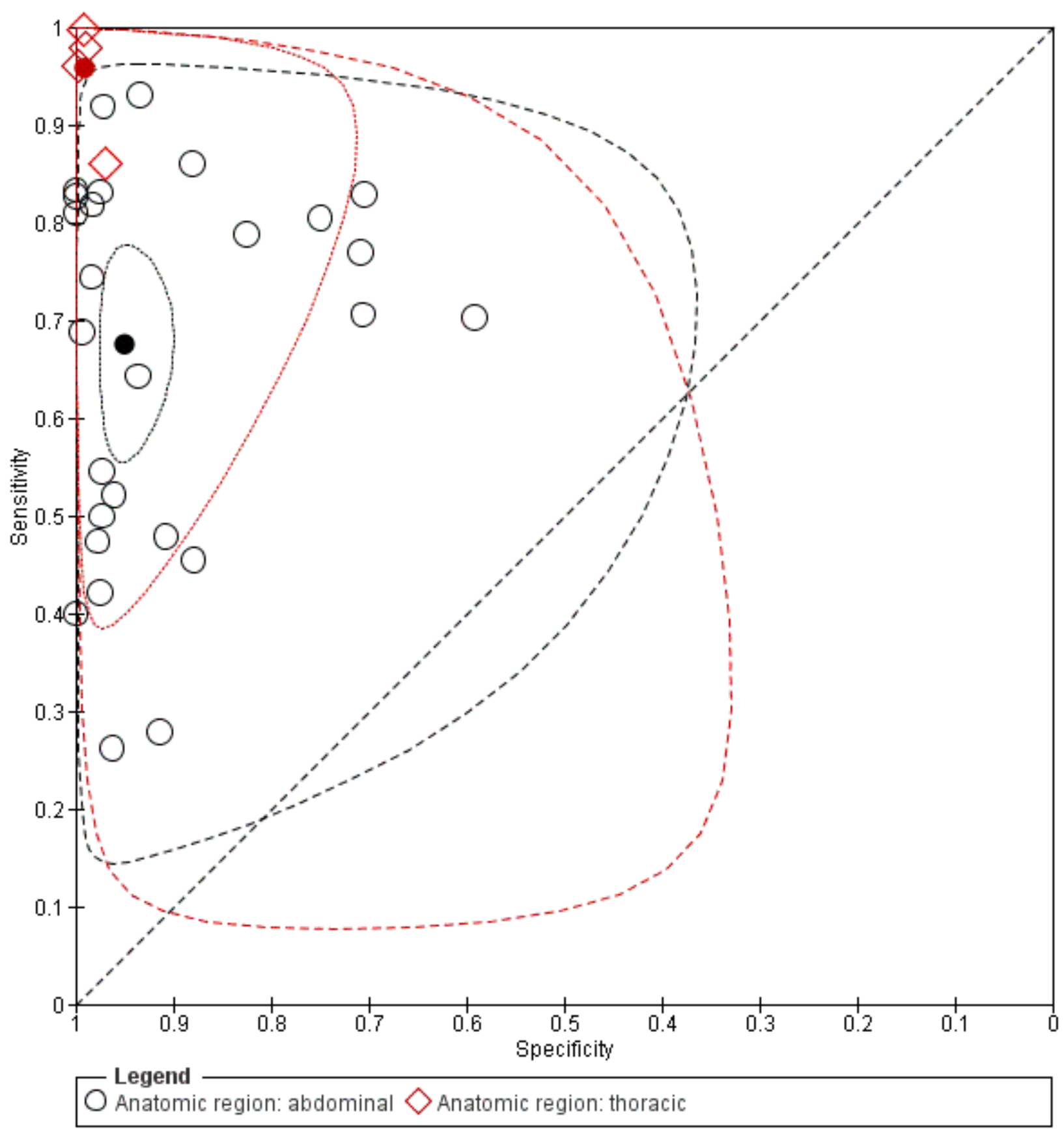

\section{Sensitivity analysis}

\section{a. Effect of study quality}

We performed sensitivity analyses to investigate the effect of study quality on diagnostic accuracy estimates separately for each of the QUADAS-2 key domains. Study quality did not have a substantial effect on either sensitivity or specificity estimates in any of the four domains. Sensitivity and specificity estimates for studies with low risk of bias were as follows: patient selection (9 studies) 0.75 (95\% $\mathrm{Cl} 0.63$ to 0.85 ) and 0.93 ( $95 \% \mathrm{Cl} 0.83$ to 0.98 ), index test (22 studies) 0.77 (95\% $\mathrm{Cl} 0.66$ to 0.85$)$ and $0.97(95 \% \mathrm{Cl} 0.94$ to 0.98$)$, reference standard ( 11 studies) 0.77 ( $95 \% \mathrm{Cl} 0.60$ to 0.89 ) and $0.97(95 \% \mathrm{Cl} 0.93$ to 0.99 ), flow and timing ( 30 studies) 0.74 ( $95 \% \mathrm{Cl} 0.65$ to 0.81 ) and 0.96 ( $95 \% \mathrm{Cl} 0.93$ to 0.98 ). 


\section{b. Effect of two independent reviewers within original studies}

In two original studies (Benya 2000; Ghafouri 2016), sonograms and CT examinations were evaluated independently by two trialists, resulting in two different accuracy values for each reviewer. In order to analyse the influence of reviewers' decision on pooled accuracy estimates, we added both reviewers' lower accuracy estimates (Main analysis set) to one set and both reviewers' higher accuracy estimates to another set (Sensitivity analysis set) and assessed the differences; we detected no difference in either diagnostic accuracy estimate. The summary estimates of sensitivity and specificity along with $\mathrm{Cls}$ were identical in both sets with sensitivity estimates of $0.74(95 \% \mathrm{Cl} 0.65$ to 0.81$)$ and specificity estimates of 0.96 (95\% $\mathrm{Cl} 0.94$ to 0.98 ). The positive and negative LRs differed marginally with $20.9(95 \% \mathrm{Cl} 12.0$ to 36.5$)$ and $0.27(95 \% \mathrm{Cl} 0.20$ to 0.37$)$ in the Main analysis set compared with $20.8(95 \% \mathrm{Cl} 11.8$ to 36.8$)$ and 0.27 $(95 \% \mathrm{Cl} 0.20$ to 0.37$)$ in the Sensitivity analysis set.

\section{c. Effect of patient age}

When including only studies with patients under 18 years of age, sensitivity and specificity values were lower than in the analysis that included adults. In the 10 paediatric studies with 1384 participants, sensitivity was estimated at $0.62(95 \% \mathrm{Cl} 0.47$ to 0.75 ) and specificity at $0.91(95 \% \mathrm{Cl} 0.81$ to 0.96$)$ (see Summary of findings 1). Positive LR with 6.9 (95\% $\mathrm{Cl} 2.5$ to 18.8), PPV with 0.76 ( $95 \% \mathrm{Cl} 0.53$ to 0.89$)$, and NPV with 0.84 (95\% Cl 0.77 to 0.90$)$ were lower, and negative LR was higher with 0.42 (95\% $\mathrm{Cl} 0.26$ to 0.65 ) than in the complete set of studies. In a virtual cohort of 1000 children having sustained blunt trauma, thoracoabdominal injuries would be missed in 118 cases (compared to 73 in the overall cohort), and 62 children would be falsely diagnosed as having sustained injuries (compared to 29 in the overall cohort).

\section{DISCUSSION}

\section{Summary of main results}

(See Summary of findings 1 )

In this systematic review, we included 34 studies with 8635 participants that evaluated the diagnostic accuracy of point-of-care sonography (POCS) for diagnosing thoracoabdominal injuries in patients with blunt trauma. Summary estimates of sensitivity and specificity were 0.74 (95\% confidence interval $(\mathrm{Cl}) 0.65$ to 0.81 ) and 0.96 (95\% Cl 0.94 to 0.98 ). Corresponding positive and negative likelihood ratios were $18.5(95 \% \mathrm{Cl} 10.8$ to 40.5$)$ and 0.27 (95\% $\mathrm{Cl} 0.19$ to 0.37 ), respectively. There was no threshold effect. We judged risk of bias as largely unclear due to insufficient information, especially in terms of patient selection and reference standard domains.

There was significant heterogeneity in both sensitivity and specificity across studies, which was partly explained by patient age, type of injury, and target condition. In children, the pooled sensitivity of POCS was 0.63 ( $95 \% \mathrm{Cl} 0.46$ to 0.77 ), compared to 0.78 $(95 \% \mathrm{Cl} 0.69$ to 0.84$)$ in an adult or mixed population. Associated specificity was $0.91(95 \% \mathrm{Cl} 0.81$ to 0.96$)$ and $0.97(95 \% \mathrm{Cl} 0.96$ to 0.99 ). Pair-wise comparisons for both sensitivity and specificity yielded $P$ values less than 0.0001 . Taking into account the rather large number of studies in both groups (i.e. 10 paediatric studies versus 24 non-paediatric studies), this indicates a real difference between both groups that cannot be explained by chance or other characteristics. For abdominal trauma, POCS had a sensitivity of
$0.68(95 \% \mathrm{Cl} 0.59$ to 0.75$)$ and a specificity of $0.95(95 \% \mathrm{Cl} 0.92$ to 0.97 ). For chest injuries, sensitivity and specificity were 0.96 (95\% Cl 0.88 to 0.99 ) and 0.99 (95\% $\mathrm{Cl} 0.97$ to 1.00$)$, respectively. However, only four studies targeted chest injuries exclusively, none of which enrolled children, for whom accuracy estimates appear to be lower generally. The individual target condition mainly affected specificity estimates, with a specificity of 0.97 (95\% Cl 0.96 to 0.99$)$ for evaluations limited to free fluid/air and a specificity of 0.88 (95\% $\mathrm{Cl} 0.70$ to 0.96 ) for complete assessments that also included direct signs of organ damage.

Summary estimates of sensitivity and specificity remained similar in studies at low risk of bias across all four domains (ranging from 0.74 to 0.77 and 0.93 to 0.97 , respectively). When only children were included, summary estimates were lower compared to the main analysis, with a sensitivity of $0.62(95 \% \mathrm{Cl} 0.47$ to 0.75$)$ and a specificity of 0.91 ( $95 \% \mathrm{Cl} 0.81$ to 0.96$)$.

In a virtual cohort of 1000 patients, assuming the observed median prevalence of thoracoabdominal trauma of $28 \%$, POCS would miss 73 patients with injuries, and falsely suggest the presence of injuries in another 29 patients. In a children-only cohort, POCS would miss 118 patients with injuries, and falsely suggest the presence of injuries in another 62 patients.

\section{Strengths and weaknesses of the review}

We performed a comprehensive literature search in major electronic databases using a reproducible retrieval strategy. With 2296 screened records and 34 eligible studies, we are confident the data set constitutes, at minimum, a representative sample and, at best, a complete set of studies investigating the diagnostic accuracy of POCS in patients with blunt trauma. We included diagnostic test accuracy (DTA) filters in our Ovid MEDLINE and Ovid Embase search strategies as suggested by the Information Specialist of the Cochrane Injuries Group and in adherence with the protocol. Methodological search filters are generally used to cut down large numbers of primary studies and to focus the search on the most relevant citations (Lefebvre 2017). Current recommendations do not support the use of methodological filters, as they may impair sensitivity and precision (Beynon 2013). Although we are not aware of any major accuracy study missed by our search algorithm, the use of a methodological search filter might represent a potential limitation in this review.

The relatively large number of investigations allowed for pooling summary estimates of sensitivity and specificity, and for exploring potential sources of heterogeneity. Using tailored review-specific signalling questions in the QUADAS-2 tool allowed us to perform a custom-made assessment of the methodological quality of all included studies. Unfortunately, assessment of the methodological quality was impeded due to considerable under-reporting in original studies. We investigated the influence of poor study quality by conducting sensitivity analyses separately for each risk-ofbias domain, which showed that study quality did not influence diagnostic accuracy estimates markedly.

There was substantial heterogeneity between studies, visible by the rather wide 95\% prediction region in Figure 5 . As there are more patients with than without the target condition, the $95 \%$ prediction region for sensitivity ( 0.14 to 0.98 ) was larger than that for specificity $(0.42$ to 1.00$)$. However, we were able to explain heterogeneity partly by study characteristics such as participants' 
age, target condition, and type of injury. The performance of POCS in children remains controversial (Holmes 2007). The lower specificity observed in this review may be explained by the disproportionately larger number of complete assessments of abdominal injuries in paediatric compared to adult studies.

The higher sensitivity and specificity of POCS in studies examining only the thorax in comparison with studies focusing on the abdomen is in agreement with previous reviews (Alrajab 2013; Alrajhi 2012; Ding 2011; Ebrahimi 2014). In our review, an evaluation of both free-fluid and organ injuries by POCS resulted in lower specificity than a complete evaluation, which is in agreement with published results (e.g. Poletti 2003).

Due to missing information, we were unable to explore some characteristics (i.e. environment, operators' expertise and background, hardware, test thresholds) as sources of heterogeneity. Only two studies described the handling of inconclusive results: in lqbal 2014, inconclusive results were handled as positive test results, and in Dolich 2001, indeterminate results were excluded from sensitivity and specificity calculations, and the percentage of indeterminate results was only $1 \%$. We do not expect the generally low number of inconclusive test results in the primary studies to affect our results. We had to modify our original categorisation of reference standards and participant age to investigate heterogeneity. Since computed tomography (CT) was used as a reference test in every single study, we compared the diagnostic accuracy of POCS to CT and CT plus laparotomy. Given the small number of studies including adults exclusively, we decided to compare children-only cohorts against studies including adults or a mixed-age population. We preferred this approach over splitting data into two parts based on participants' median or mean age.

We classified participants as test positive if any one of the target conditions was detected, irrespective of the fact that target conditions could differ between index test and reference standard. The majority of original studies (i.e. 25 of 34) used similar target conditions for index test and reference standard, and thus did not cause a mismatch. However, non-transparent reporting in the remaining studies prevented us from correlating the source of bleeding between both diagnostic procedures, and may potentially result in a mismatch regarding the target condition. We do not presume a substantial mismatch here, however an effect on test accuracy estimates cannot be excluded.

We restricted suitable reference standards to predefined imaging or invasive tests (i.e. CT, magnetic resonance imaging (MRI), laparotomy, laparoscopy, thoracotomy, thoracoscopy, autopsy), which ensured accurate estimation of sensitivities and specificities of POCS as an index test. However, we were unable to detect investigations that used MRI, laparoscopy, thoracotomy, or thoracoscopy as the single reference standard as specified by our inclusion and exclusion criteria, thus we could not evaluate the diagnostic accuracy of POCS compared to these diagnostic techniques. As a consequence, the diagnostic accuracy of POCS mainly refers to $C T$ as a comparator rather than any other reference standard, and may thus limit the generalisability of this review.

\section{Applicability of findings to the review question}

In order to generate clinically realistic and relevant evidence, we kept our inclusion criteria fairly broad. Consequently, individual study and participant characteristics varied substantially, for example in terms of age, affected body region, target conditions, operators' expertise, hardware specification, etc. Unsurprisingly, while this variation led to marked heterogeneity in both sensitivity and specificity estimates between studies, it also enabled us to compare accuracy estimates across various settings and POCS applications.

We assessed concerns about applicability in participant selection, index test, and reference standards by using tailored questions in the QUADAS-2 tool. Of the 34 included studies, 11 were associated with low concern about applicability (Benya 2000; Blaivas 2005; Coley 2000; Emery 2001; Ghafouri 2016; Kendall 2009; Nandipati 2011; Ojaghi 2014; Talari 2015; Todd Miller 2003; Zhang 2006). In the patient selection and index test domains, 29 studies showed low concerns about applicability. In patient selection, we assigned high concerns to five studies because of restricted inclusion criteria (i.e. only pelvic fractures (Friese 2007; Verbeek 2014); only minor, Menichini 2015, or only major trauma, Corbett 2000; or with limited organ lesions only (Kärk 2012)). We judged five studies to have unclear ratings in the index test domain owing to missing information about body areas examined (Calder 2017; Clevert 2008; Valentino 2010; Wong 2014; Zhou 2012). The conditional use of reference standards depending on the results of clinical observation, ultrasound examination, or participants' haemodynamic stability led to 16 high applicability rating concerns in the reference standard domain.

In summary, we rated $85 \%$ of all included studies as being of low concern for applicability in the patient selection and index test domains, whereas we rated only $47 \%$ of studies as of low concern in the reference standard domains. While the included spectrum of participants in this review may appropriately reflect the intended population, and the index tests used in the included studies may not differ considerably from those in clinical practice, the spectrum of reference standards may not correspond completely to the whole range of tests actually used in the setting of thoracoabdominal trauma.

\section{AUTHORS' CONCLUSIONS}

\section{Implications for practice}

Following the 'treat first what kills first' principle, any active non-compressible bleeding in the major body cavities and retroperitoneum represents a priority condition to be addressed immediately (e.g. by pelvic stabilisation, haemostatic transfusion, tranexamic acid, etc.). As damage-control resuscitation often needs a substantial number of precious packed red blood and fresh frozen plasma units, the high specificity of point-of-care sonography (POCS) $(0.96,95 \%$ confidence interval $(\mathrm{Cl}) 0.94$ to 0.98) may avoid a waste of resources (which is of particular importance in mass casualties), overtreatment, and unnecessary invasive procedures, as false-positive findings are very unlikely (with 266 false positives (i.e. 3.1\%) out of 8635 individuals). Also, the accuracy of ultrasonography for identifying chest injuries such as pneumothorax with a sensitivity calculated at $0.96(95 \% \mathrm{Cl} 0.88$ to 0.99$)$ and a specificity calculated at $0.99(95 \% \mathrm{Cl} 0.97$ to 1.00$)$ based on four studies is remarkable and may replace traditional posteroanterior radiographs.

However, despite the advantage of the high specificity of ultrasonography, multiprofessional trauma care teams need to be 
aware that a negative examination bears a relevant risk of being false negative (i.e. negative predictive value $(0.90,95 \% \mathrm{Cl} 0.87$ to $0.93)$ ). Where there is a high prior probability of thoracoabdominal trauma (e.g. because of the injury mechanism), a negative scan may also be caused by centralised circulation and limited arterial perfusion of injured solid organs like the liver or spleen.

Again, it remains important to consider the individual clinical scenario when interpreting POCS findings. While positive results will be almost always trustworthy and should prompt bleeding control measures, negative scans must be confirmed by a reference test like computed tomography (CT), or, in the case of limited resources, by sequential sonograms and clinical observation. This is of particular importance in paediatric trauma, where the sensitivity of POCS is extremely poor $(0.62,95 \% \mathrm{Cl} 0.47$ to 0.75$)$, potentially resulting in 118 children with missed injuries in a cohort of 1000 children with suspected blunt thoracoabdominal trauma. These accuracy patterns are probably a signature feature of POCS that cannot be overcome even by state-of-the art equipment.

\section{Implications for research}

In high-income countries, the availability of fast CT scanners right at or close to the trauma bay, together with wholebody scanning protocols and dose-reducing algorithms, have substantially reduced the clinical importance of POCS in routine trauma care. Additional studies on the accuracy of this technology to detect abdominal injuries may thus have little impact on care processes in all age groups. More accurate reporting of individual study characteristics (e.g. selection of participants, examiner's experience) would help to evaluate potential sources of heterogeneity in the diagnosis of blunt thoracoabdominal trauma better, and to assess the risk of bias. Nonetheless, more and robust data from larger, confirmatory studies using CT as the ultimate reference test are required to define the role of POCS for detecting pneumothorax and haematothorax and facilitating early tube thoracostomy. Studies determining the accuracy and utility of POCS in mass casualty and low- and middle-income countries are needed, however guaranteeing consistent confirmation of POCS findings by objective reference tests in these settings will be challenging.

\section{ACKNOWLEDGEMENTS}

We thank Emma Sydenham and Elizabeth Royle, editors of the Cochrane Injuries Group, for their constructive feedback in drafting the protocol. We also thank Sarah Dawson, Information Specialist of the Cochrane Injuries Group, for her help in developing the search strategy.

This project was supported by the UK National Institute for Health Research (NIHR), through Cochrane Infrastructure funding to the Cochrane Injuries Group. The views and opinions expressed are those of the authors and do not necessarily reflect those of the Systematic Reviews Programme, NIHR, National Health Service (NHS), or the Department of Health. 


\section{REFERE N CES}

\section{References to studies included in this review}

Becker 2010 \{published data only\}

Becker A, Lin G, McKenney MG, Marttos A, Schulman Cl. Is the FAST exam reliable in severely injured patients?. Injury 2010;41(5):479-83.

\section{Benya 2000 \{published data only\}}

Benya EC, Lim-Dunham JE, Landrum O, Statter M. Abdominal sonography in examination of children with blunt abdominal trauma. American Journal of Roentgenology 2000;174(6):1613-6.

\section{Blaivas 2005 \{published data only\}}

Blaivas M, Lyon M, Duggal S. A prospective comparison of supine chest radiography and bedside ultrasound for the diagnosis of traumatic pneumothorax. Academic Emergency Medicine 2005;12(9):844-9.

\section{Calder 2017 \{published data only\}}

Calder BW, Vogel AM, Zhang J, Mauldin PD, Huang EY, Savoie KB, et al. Focused Assessment with Sonography for Trauma (FAST) in children following blunt abdominal trauma: a multiinstitutional analysis. Journal of Trauma and Acute Care Surgery 2017;83(2):218-24

\section{Catalano 2009 \{published data only\}}

Catalano O, Aiani L, Barozzi L, Bokor D, De Marchi A, Faletti C, et al. CEUS in abdominal trauma: multi-center study. Abdominal Imaging 2009;34(2):225-34.

\section{Cheung 2012 \{published data only\}}

Cheung KS, Wong HT, Leung LP, Tsang TC, Leung GK. Diagnostic accuracy of Focused Abdominal Sonography for Trauma in blunt abdominal trauma patients in a trauma centre of Hong Kong. Chinese Journal of Traumatology 2012;15(5):273-8.

\section{Clevert 2008 \{published data only\}}

Clevert DA, Weckbach S, Minaifar N, Clevert DA, Stickel M, Reiser M. Contrast-enhanced ultrasound versus MS-CT in blunt abdominal trauma. Clinical Hemorheology and Microcirculation 2008:39(1-4):155-69.

\section{Coley 2000 \{published data only\}}

Coley BD, Mutabagani KH, Martin LC, Zumberge N, Cooney DR, Caniano DA, et al. Focused abdominal sonography for trauma (FAST) in children with blunt abdominal trauma. Journal of Trauma and Acute Care Surgery 2000;48(5):902-6.

\section{Corbett 2000 \{published data only\}}

Corbett SW, Andrews HG, Baker EM, Jones WG. ED evaluation of the pediatric trauma patient by ultrasonography. American Journal of Emergency Medicine 2000;18(3):244-9.

\section{Dolich 2001 \{published data only\}}

Dolich MO, McKenney MG, Varela JE, Compton RP, McKenney KL, Cohn SM. 2,576 ultrasounds for blunt abdominal trauma. Journal of Trauma and Acute Care Surgery 2001;50(1):108-12.
Emery 2001 \{published data only\}

Emery KH, McAneney CM, Racadio JM, Johnson ND, Evora DK, Garcia VF. Absent peritoneal fluid on screening trauma ultrasonography in children: a prospective comparison with computed tomography. Journal of Pediatric Surgery 2001;36(4):565-9.

\section{Fox 2011 \{published data only\}}

Fox JC, Boysen M, Gharahbaghian L, Cusick S, Ahmed SS, Anderson CL, et al. Test characteristics of focused assessment of sonography for trauma for clinically significant abdominal free fluid in pediatric blunt abdominal trauma. Academic Emergency Medicine 2011;18(5):477-82.

\section{Friese 2007 \{published data only\}}

Friese RS, Malekzadeh S, Shafi S, Gentilello LM, Starr A. Abdominal ultrasound is an unreliable modality for the detection of hemoperitoneum in patients with pelvic fracture. Journal of Trauma Injury, Infection and Critical Care 2007;63(1):97-102.

\section{Ghafouri 2016 \{published data only\}}

Ghafouri HB, Zare M, Bazrafshan A, Modirian E, Farahmand S, Abazarian N. Diagnostic accuracy of emergency-performed focused assessment with sonography for trauma (FAST) in blunt abdominal trauma. Electronic Physician 2016;8(9):2950-3.

\section{Hsu 2007 \{published data only\}}

Hsu JM, Joseph AP, Tarlinton LJ, Macken L, Blome S. The accuracy of focused assessment with sonography in trauma (FAST) in blunt trauma patients: experience of an Australian major trauma service. Injury 2007;38(1):71-5.

Iqbal 2014 \{published data only\} Iqbal Y, Taj MN, Ahmed A, Ur Rehman Z, Akbar Z. Validity of the fast scan for diagnosis of intraabdominal injury in blunt abdominal trauma. Journal of Ayub Medical College, Abbottabad 2014;26(1):52-6.

\section{Kärk 2012 \{published data only\}}

Kärk Nielsen S, Ewertsen C, Svendsen LB, Hillingso JG, Nielsen MB. Focused assessment with sonography for trauma in patients with confirmed liver lesions. Scandinavian Journal of Surgery 2012;101(4):287-91.

\section{Kendall 2009 \{published data only\}}

Kendall JL, Faragher J, Hewitt GJ, Burcham G, Haukoos JS. Emergency department ultrasound is not a sensitive detector of solid organ injury. Western Journal of Emergency Medicine 2009;10(1):1-5.

\section{Kumar 2015 \{published data only\}}

Kumar S, Bansal VK, Muduly DK, Sharma P, Misra MC, Chumber S, et al. Accuracy of Focused Assessment with Sonography for Trauma (FAST) in blunt trauma abdomen. Indian Journal of Surgery 2015;77(2):393-7. 
McElveen 1997 \{published data only\}

McElveen TS, Collin GR. The role of ultrasonography in blunt abdominal trauma: a prospective study. American Surgeon 1997;63(2):184-8.

\section{McKenney 1994 \{published data only\}}

McKenney M, Lentz K, Nunez D, Sosa JL, Sleeman D, Axelrad A, et al. Can ultrasound replace diagnostic peritoneal lavage in the assessment of blunt trauma?. Journal of Trauma and Acute Care Surgery 1994;37(3):439-41.

\section{Menichini 2015 \{published data only\}}

Menichini G, Sessa B, Trinci M, Galluzzo M, Miele V. Accuracy of contrast-enhanced ultrasound (CEUS) in the identification and characterization of traumatic solid organ lesions in children: a retrospective comparison with baseline US and CE-MDCT. Radiologia Medica 2015;120(11):989-1001.

Nandipati 2011 \{published data only\}

Nandipati KC, Allamaneni S, Kakarla R, Wong A, Richards N, Satterfield J, et al. Extended focused assessment with sonography for trauma (EFAST) in the diagnosis of pneumothorax: experience at a community based level I trauma center. Injury 2011;42(5):511-4.

\section{Ojaghi 2014 \{published data only\}}

Ojaghi Haghighi SH, Adimi I, Shams Vahdati S, Sarkhoshi Khiavi R. Ultrasonographic diagnosis of suspected hemopneumothorax in trauma patients. Trauma Monthly 2014;19(4):e17498.

\section{Smith 2010 \{published data only\}}

Smith ZA, Postma N, Wood D. FAST scanning in the developing world emergency department. South African Medical Journal 2010;100(2):105-8.

\section{Soudack 2004 \{published data only\}}

Soudack M, Epelman M, Maor R, Hayari L, Shoshani G, HeymanReiss $A$, et al. Experience with focused abdominal sonography for trauma (FAST) in 313 pediatric patients. Journal of Clinical Ultrasound 2004;32(2):53-61.

\section{Talari 2015 \{published data only\}}

Talari H, Moussavi N, Abedzadeh-Kalahroudi M, Atoof F, Abedini A. Correlation between intra-abdominal free fluid and solid organ injury in blunt abdominal trauma. Archives of Trauma Research 2015;4(3):e29184.

\section{Todd Miller 2003 \{published data only\}}

Todd Miller M, Pasquale MD, Bromberg WJ, Wasser TE, Cox J, Rozycki GS, et al. Not so fast. Journal of Trauma Injury, Infection and Critical Care 2003;54(1):52-60.

\section{Tso 1992 \{published data only\}}

Tso P, Rodriguez A, Cooper C, Militello P, Mirvis S, Badellino MM, et al. Sonography in blunt abdominal trauma: a preliminary progress report. Journal of Trauma and Acute Care Surgery 1992;33(1):39-43.

\section{Valentino 2010 \{published data only\}}

Valentino M, De Luca C, Galloni SS, Branchini M, Modolon C, Pavlica P, et al. Contrast-enhanced US evaluation in patients with blunt abdominal trauma. Journal of Ultrasound 2010;13(1):22-7.

\section{Verbeek 2014 \{published data only\}}

Verbeek DO, Zijlstra IA, van der Leij C, Ponsen KJ, van Delden OM, Goslings JC. The utility of FAST for initial abdominal screening of major pelvic fracture patients. World Journal of Surgery 2014;38(7):1719-25.

\section{Wong 2014 \{published data only\}}

Wong E, Ngo ASY, Wee JCP, Lee JMH. Focused assessment with sonography in trauma (FAST): experience of a tertiary hospital in Southeast Asia. Hong Kong Journal of Emergency Medicine 2014;21(4):230-6.

\section{Zhang 2006 \{published data only\}}

Zhang M, Liu Z, Yang J, Gan J, Xu S, You X, et al. Rapid detection of pneumothorax by ultrasonography in patients with multiple trauma. Critical Care 2006;10(4):R112.

\section{Zhou 2012 \{published data only\}}

Zhou J, Huang J, Wu H, Jiang H, Zhang H, Prasoon P, et al. Screening ultrasonography of 2,204 patients with blunt abdominal trauma in the Wenchuan earthquake. Journal of Trauma and Acute Care Surgery 2012;73(4):890-4.

\section{References to studies excluded from this review}

Abdulrahman 2015 \{published data only\}

Abdulrahman Y, Musthafa S, Hakim SY, Nabir S, Qanbar A, Mahmood I, et al. Utility of extended FAST in blunt chest trauma. World Journal of Surgery 2015;39(1):172-8.

\section{Ala 2016 \{published data only\}}

Ala AR, Pouraghaei M, Vahdati SS, Taghizadieh A, Moharamzadeh P, Arjmandi H. Diagnostic accuracy of focused assessment with sonography for trauma in the emergency department. Trauma Monthly 2016;21(4):e21122.

\section{Arrillaga 1999 \{published data only\}}

Arrillaga A, Graham R, York JW, Miller RS. Increased efficiency and cost-effectiveness in the evaluation of the blunt abdominal trauma patient with the use of ultrasound. American Journal of Surgery 1999;65(1):31-5.

\section{Beck-Razi 2007 \{published data only\}}

Beck-Razi N, Fischer D, Michaelson M, Engel A, Gaitini D. The utility of focused assessment with sonography for trauma as a triage tool in multiple-casualty incidents during the second Lebanon War. Journal of Ultrasound in Medicine 2007;26(9):1149-56.

\section{Behboodi 2016 \{published data only\}}

Behboodi F, Mohtasham-Amiri Z, Masjedi N, Shojaie R, Sadri P. Outcome of blunt abdominal traumas with stable hemodynamic and positive FAST findings. Emergency 2016;4(3):136-9. 
Brooks 2004a \{published data only\}

Brooks A, Davies B, Smethhurst M, Connoly J. Prospective evaluation of non-radiologist performed emergency abdominal ultrasound for haemoperitoneum. Emergency Medicine Journal 2004;21(5):580-1.

\section{Brooks 2004b \{published data only\}}

Brooks A, Davies B, Smethhurst M, Connoly J. Emergency ultrasound in the acute assessment of haemothorax. Emergency Medicine Journal 2004;21:44-6.

\section{Brown 2001 \{published data only\}}

Brown MA, Casola G, Sirlin CB, Patel NY, Hoyt DB. Blunt abdominal trauma: screening US in 2693 patients. Radiology 2001;218(2):352-8

\section{Byars 2013 \{published data only\}}

Byars D, Devine A, Maples C, Yeats A, Greene K. Physical examination combined with focused assessment with sonography for trauma examination to clear hemodynamically stable blunt abdominal trauma patients. American Journal of Emergency Medicine 2013;31:1525-34.

\section{Cook 2015 \{published data only\}}

Cook MR, Holcomb J, Rahbar M, Fox E, Alarcon L, Bulger E, et al. An abdominal computed tomography may be safe in selected hypotensive trauma patients with positive focused assessment with sonography in trauma examination. American Journal of Surgery 2015;209:834-40.

\section{Coskun 2011 \{published data only\}}

Coskun F, Akinci E, Ceyhan M, Sahin Kavakli H. Our new stethoscope in the Emergency Department: handheld ultrasound. Ulus Trauma Acil Cerrahi Derg [Turkish Journal of Trauma \& Emergency Surgery: TJTES] 2011;17(6):488-92.

\section{Dan 2010 \{published data only\}}

Dan D, Mingsong L, Jie T, Xiaobo W, Thong C, Yan L, et al. Ultrasonographic applications after mass casualty incident caused by Wenchuan Earthquake. Journal of Trauma 2010;68:1417-20.

\section{Deunk 2010 \{published data only\}}

Deunk J, Brink M, Dekker H, Kool D, Blickman J, van Vugt A, et al. Predictors for the selection of patients for abdominal CT after blunt trauma. Annals of Surgery 2010;251:512-20.

\section{Donmez 2011 \{published data only\}}

Donmez H, Tokmak T, Yildirim A, Buyukoglan H, Ozturk M, Ayaz Ü, et al. Should bedside sonography be used first to diagnose pneumothorax secondary to blunt trauma?. Journal of Clinical Ultrasound 2011;40:142-6.

\section{Faruque 2013 \{published data only\}}

Faruque AV, Qazi SH, Khan MA, Akhtar W, Majeed A. Focused abdominal sonography for trauma (FAST) in blunt paediatric abdominal trauma. Journal of the Pakistan Medical Association 2013;63(3):361-4.

\section{Hamada 2016 \{published data only\}}

Hamada SR, Delhaye N, Kerever S, Harrois A, Duranteau J. Integrating eFast in the initial management of stable trauma patients: the end of plain film radiography. Annals of Intensive Care 2016;6:62.

\section{Helling 2007 \{published data only\}}

Helling TS, Wilson J, Augustosky K. The utility of focused abdominal ultrasound in blunt abdominal trauma: a reappraisal. American Journal of Surgery 2007;194(6):728-33.

\section{Heyn 2008 \{published data only\}}

Heyn J, Ladurner R, Ozimek A, Bürklein D, Huber-Wagner SM, Hallfeldt KK, et al. Diagnosis and pre-operative management of multiple injured patients with explorative laparotomy because of blunt abdominal trauma. European Journal of Medical Research 2008;13:517-24.

\section{Holmes 2012 \{published data only\}}

Holmes G, Romero J, Waman K, Diaz G. Fast enough? A validation study for focused assessment with sonography for trauma ultrasounds in a level II trauma center. American Surgeon 2012;78:1038-40.

\section{Hyacinthe 2012 \{published data only\}}

Hyacinthe A, Broux C, Francony G, Genty C, Bouzat P, Jacqout $C$, et al. Diagnostic accuracy of ultrasound in the acute assessment of common thoracic lesions after trauma. Chest 2012;141(5):1177-83.

\section{Ianniello 2014 \{published data only\}}

Ianniello S, Giacomo VD, Sessa B, Miele V. First-line sonographic diagnosis of pneumothorax in major trauma: accuracy of e-FAST and comparison with multidetector computed tomography. $L a$ Radiologia Medica 2014;119(9):674-80.

\section{Ingeman 1996 \{published data only\}}

Ingeman JE, Plewa MC, Okasinski RE, King RW, Knotts EB. Emergency physician use of ultrasonography in blunt abdominal trauma. Academic Emergency Medicine 1996;3:931-7.

\section{Jalli 2009 \{published data only\}}

Jalli R, Kamalzadeh N, Lotfi M, Farahangiz S, Salehipour M. Accuracy of sonography in detection of renal injuries caused by blunt abdominal trauma: a prospective study. Ulus Trauma Acil Cerrahi Derg [Turkish Journal of Trauma \& Emergency Surgery: TJTES] 2009;15(1):23-7.

\section{Kaya 2015 \{published data only\}}

Kaya S, Cevik A, Acar N, Döner E, Sivrikoz C, Özkan R. A study on the evaluation of pneumothorax by imaging methods in patients presenting to the emergency department for blunt thoracic trauma. Ulus Trauma Acil Cerrahi Derg [Turkish Journal of Trauma \& Emergency Surgery: TJTES] 2015;21(5):366-72.

\section{Kern 1997 \{published data only\}}

Kern SJ, Smith RS, Fry WR, Helmer SD, Reed JA, Chang FC. Sonographic examination of abdominal trauma by senior surgical residents. American Surgeon 1997;63(8):669-74. 
Kirkpatrick 2002 \{published data only\}

Kirkpatrick A, Simons R, Brown R, Nicolaou S, Dulchavsky S. The handheld FAST: experience with hand-held trauma sonography in a level-I urban trauma center. Injury 2002;33:303-8.

\section{Kirkpatrick 2004 \{published data only\}}

Kirkpatrick AW, Sirois M, Laupland KB, Liu D, Rowan K, Ball CG. Hand-held thoracic sonography for detecting post-traumatic pneumothoraces: the extended focused assessment with sonography for trauma (EFAST). Journal of Trauma and Acute Care Surgery 2004;57:288-95.

\section{Kirkpatrick 2005 \{published data only\}}

Kirkpatrick A, Sirois M, Laupland K, Goldstein L, Brown D, Simons R, et al. Prospective evaluation of handheld focused abdominal sonography for trauma (FAST) in blunt abdominal trauma. Canadian Journal of Surgery 2005;48(6):453-60.

\section{Krupnick 1997 \{published data only\}}

Krupnick AS, Teitelbaum DH, Geiger JD, Strouse PJ, Cox CS, Blane CE, et al. Use of abdominal ultrasonography to assess pediatric splenic trauma. Annals of Surgery 1997;225(4):408-14.

\section{Ku 2013 \{published data only\}}

Ku BS, Fields JM, Carr B, Everett WW, Gracias VH, Dean AJ. Clinician-performed bedside ultrasound for the diagnosis of traumatic pneumothorax. Western Journal of Emergency Medicine 2013;14(2):103-8.

\section{Kumar 2014 \{published data only\}}

Kumar S, Kumar A, Joshi M, Rathi V. Comparison of diagnostic peritoneal lavage and focused assessment by sonography in trauma as an adjunct to primary survey in torso trauma: a prospective randomized clinical trial. Ulus Trauma Acil Cerr Derg [Turkish Journal of Trauma \& Emergency Surgery: TJTES] 2014;20(2):101-6.

\section{Lichtenstein 2005 \{published data only\}}

Lichtenstein D, Meziere G, Lascols N, Biderman P, Courret J, Gepner A, et al. Ultrasound diagnosis of occult pneumothorax. Critical Care Medicine 2005;33(6):1231-8.

\section{Matsumoto 2016 \{published data only\}}

Matsumoto S, Sekine K, Funabiki T, Orita T, Shimizu M, Hayashida K, et al. Diagnostic accuracy of oblique chest radiograph for occult pneumothorax: comparison with ultrasonography. World Journal of Emergency Surgery 2016;11:5.

\section{Mihalik 2012 \{published data only\}}

Mihalik JE, Smith RS, Toevs CC, Putnam AT, Foster JE. The use of contrast-enhanced ultrasound for the evaluation of solid abdominal organ injury in patients with blunt abdominal trauma. Journal of Trauma and Acute Care Surgery 2012;73(5):1100-5.

\section{Moylan 2007 \{published data only\}}

Moylan M, Newgard C, Ma O, Sabbaj A, Rogers T, Douglass R. Association between a positive ED FAST examination and therapeutic laparotomy in normotensive blunt trauma patients. Journal of Emergency Medicine 2007;33(3):265-71.
Mumtaz 2016 \{published data only\}

Mumtaz U, Zahur Z, Chaudhry M, Warraich R. Bedside ultrasonography: a useful tool for traumatic pneumothorax. Journal of the College of Physicians and Surgeons Pakistan 2016;26(6):459-62.

\section{Nagarsheth 2011 \{published data only\}}

Nagarsheth K, Kurek S. Ultrasound detection of pneumothorax compared with chest x-ray and computed tomography scan. American Surgeon 2011;77(4):480-3.

\section{Natarajan 2010 \{published data only\}}

Natarajan B, Gupta P, Cemay S, Sorenson M, Hatzoudis G, Forse R. FAST scan: is it worth doing in hemodynamically stable blunt trauma patients?. Surgery 2010;148(4):695-701.

Pak-art 2003 \{published data only\}

Pak-art R, Sriussadaporn S, Sriussadaporn S, Vajrabukka T. The results of focused assessment with sonography for trauma performed by third year surgical residents: a prospective study. Journal of the Medical Association of Thailand 2003;86(2):344-9.

Richards 2004 \{published data only\}

Richards JR, Ormsby EL, Romo MV, Gillen MA, McGahan JP. Blunt abdominal injury in the pregnant patient: detection with US. Radiology 2004;233(2):463-70.

\section{Richardson 1997 \{published data only\}}

Richardson M, Holllman A, Davis C. Comparison of computed tomography and ultrasonographic imaging in the assessment of blunt abdominal trauma in children. British Journal of Surgery 1997;84:1144-6.

\section{Schleder 2013 \{published data only\}}

Schleder S, Dendl L, Ernstberger A, Nerlich M, Hoffstetter P, Jung $E$, et al. Diagnostic value of a hand-carried ultrasound device for free intra-abdominal fluid and organ lacerations in major trauma patients. Emergency Medicine Journal 2013;30(3):e20.

\section{Sheng 2013 \{published data only\}}

Sheng A, Dalziel P, Liteplo A, Fagenholz P, Noble V. Focused assessment with sonography in trauma and abdominal computed tomography utilization in adult trauma patients: trends over the last decade. Emergency Medicine International 2013;2013:678380.

\section{Smith 2009 \{published data only\}}

Smith C, Barrett T, Berger C, Thou C, Thurman R, Wrenn K. Prediction of blunt traumatic injury in high-acuity patients: bedside examination vs. computed tomography. American Journal of Emergency Medicine 2011;29(1):1-10.

Smith 2013 \{published data only\}

Smith Z, Wood D. Emergency focussed assessment with sonography in trauma (FAST) and haemodynamic stability. Emergency Medicine Journal 2014;31:273-7. 
Smith 2015 \{published data only\}

Smith I, Naumann D, Marsden M, Ballard M, Bowley D. Scanning and war; utility of FAST and CT in the assessment of battlefield abdominal trauma. Annals of Surgery 2015;262:389-96.

\section{Soldati 2007 \{published data only\}}

Soldati G, Testa A, Sher S, Pignataro G, La Sala M, Gentiloni Silveri N. Occult traumatic pneumothorax diagnostic accuracy of lung ultrasonography in the emergency department. Chest 2008;133:204-11.

\section{Soult 2015 \{published data only\}}

Soult M, Weireter L, Britt R, Collins J, Novosel T, Reed S, et al. Can routine trauma bay chest $\mathrm{X}$-ray be bypassed with an extended focused assessment with sonography for trauma examination?. American Surgeon 2015;81:336-40.

\section{Tajoddini 2013 \{published data only\}}

Tajoddini S, Vahdati S. Ultrasonographic diagnosis of abdominal free fluid: accuracy comparison of emergency physicians and radiologists. European Journal of Trauma and Emergency Surgery 2013;39:9-13.

\section{Tam 2005 \{published data only\}}

Tam M. Occult pneumothorax in trauma patients: should this be sought in the focused assessment with sonography for trauma examination?. Emergency Medicine Australasia 2005;17:488-93.

\section{Tas 2004 \{published data only\}}

Tas F, Ceran C, Atalar MH, Bulut S, Selbes B, Isık AO. The efficacy of ultrasonography in hemodynamically stable children with blunt abdominal trauma: a prospective comparison with computed tomography. European Journal of Radiology 2004;51:91-6.

\section{Tayal 2006 \{published data only\}}

Tayal V, Nielsen A, Jones A, Thomason M, Kellam J, Norton H. Accuracy of trauma ultrasound on major pelvic injury. Journal of Trauma and Acute Care Surgery 2006;61:1453-7.

\section{Tummers 2016 \{published data only\}}

Tummers W, Van Schuppen J, Langeveld H, Wilde J, Banderker E, Van As A. Role of focused assessment with sonography for trauma as a screening tool for blunt abdominal trauma in young children after high energy trauma. South African Journal of Surgery 2016;54(2):28-34.

\section{Tunuka 2014 \{published data only\}}

Tunuka C, Wangoda R, Bugeza S, Galukande M. Emergency sonography aids diagnostic accuracy of torso injuries: a study in a resource limited setting. Emergency Medicine International 2014;2014:978795.

\section{Valentino 2008 \{published data only\}}

Valentino M, Serra C, Pavlica P, Morselli Labate AM, Lima M, Baroncini $S$, et al. Blunt abdominal trauma: diagnostic performance of contrast enhanced US in children - initial experience. Radiology 2008;246(3):903-9.

\section{Van Diepen 2013 \{published data only\}}

Van Diepen K, Adams C, Harris M, Lin D. The sensitivity and specificity of the focused assessment with sonography for trauma in blunt abdominal injury in a level I trauma centre. Résumés Scientifique 2013;15(1):66.

Vassiliadis 2003 \{published data only\}

Vassiliadis J, Edwards R, Larcos G, Hitos K. Focused assessment with sonography for trauma patients by clinicians: initial experience and results. Emergency Medicine 2003;15:42-8.

\section{References to studies awaiting assessment}

Armstrong 2018 \{published data only\}

Armstrong LB, Mooney DP, Paltiel H, Barnewolt C, Dionigi B, Arbuthnot M, et al. Contrast enhanced ultrasound for the evaluation of blunt pediatric abdominal trauma. Journal of Pediatric Surgery 2018;53(3):548-52.

Elbaih 2017 \{published data only\}

Elbaih AH, Abu-Elela ST. Predictive value of focused assessment with sonography for trauma (FAST) for laparotomy in unstable polytrauma Egyptians patients. Chinese Journal of Traumatology 2017;20(6):323-8.

\section{Hsu 2017 \{published data only\}}

Hsu SD, Chen CJ, Chan DC, Yu JC. Senior general surgery residents can be trained to perform focused assessment with sonography for trauma patients accurately. Surgery Today 2017;47(12):1443-9.

\section{Kozaci 2018 \{published data only\}}

Kozaci N, Avci M, Ararat E, Pinarbasili T, Ozkaya M, Etli I, et al. Comparison of ultrasonography and computed tomography in the determination of traumatic thoracic injuries. American Journal of Emergency Medicine 2018 Aug 3 [Epub ahead of print]. [DOI: 10.1016/j.ajem.2018.08.002]

\section{Maximus 2018 \{published data only\}}

Maximus S, Figueroa C, Whealon M, Pham J, Kuncir E, Barrios C. eFAST for pneumothorax: real-life application in an urban level 1 center by trauma team members. American Surgeon 2018;84(2):220-4.

\section{Mumtaz 2017 \{published data only\}}

Mumtaz U, Zahur Z, Raza MA, Mumtaz M. Ultrasound and supine chest radiograph In road traffic accident patients: a reliable and convenient way to diagnose pleural effusion. Journal of Ayub Medical College Abbottabad 2017;29(4):587-90.

\section{Sauter 2017 \{published data only\}}

Sauter TC, Hoess S, Lehmann B, Exadaktylos AK, Haider DG. Detection of pneumothoraces in patients with multiple blunt trauma: use and limitations of eFAST. Emergency Medicine Journal 2017;34(9):568-72.

\section{Waheed 2018 \{published data only\}}

Waheed KB, Baig AA, Raza A, UI Hassan MZ, Khattab MA, Raza U. Diagnostic accuracy of focused assessment with sonography 
for trauma for blunt abdominal trauma in the Eastern Region of Saudi Arabia. Saudi Medical Journal 2018;39(6):598-602.

\section{Zieleskiewicz 2018 \{published data only\}}

Zieleskiewicz L, Fresco R, Duclos G, Antonini F, Mathieu C, Medam S, et al. Integrating extended focused assessment with sonography for trauma (eFAST) in the initial assessment of severe trauma: Impact on the management of 756 patients. Injury 2018;49(10):1774-80.

\section{Additional references}

\section{Alrajab 2013}

Alrajab S, Youssef AM, Akkus NI, Caldito G. Pleural ultrasonography versus chest radiography for the diagnosis of pneumothorax: review of the literature and meta-analysis. Journal of Critical Care 2013;17(5):R208.

\section{Alrajhi 2012}

Alrajhi K, Woo MY, Vaillancourt C. Test characteristics of ultrasonography for the detection of pneumothorax: a systematic review and meta-analysis. Chest 2012;141(3):703-8.

\section{Asha 2012}

Asha S, Curtis KA, Grant N, Taylor C, Lo S, Smart R, et al. Comparison of radiation exposure of trauma patients from diagnostic radiology procedures before and after the introduction of a panscan protocol. Emergency Medicine Australasia 2012;24(1):43-51

\section{Beynon 2013}

Beynon R, Leeflang MM, McDonald S, Eisinga A, Mitchell RL, Whiting $P$, et al. Search strategies to identify diagnostic accuracy studies in MEDLINE and EMBASE. Cochrane Database of Systematic Reviews 2013, Issue 9. [DOI: 10.1002/14651858.MR000022.pub3]

\section{Chapleau 2013}

Chapleau W, Al-khatib J, Haskin D, LeBlanc P, Cardenas B, Borum $S$, et al. Advanced trauma life support $\left(A T L S^{\circledast}\right)$ : the ninth edition. Journal of Trauma and Acute Care Surgery 2013;74(5):1363-6.

\section{Deeks 2005}

Deeks JJ, Macaskill P, Irwig L. The performance of tests of publication bias and other sample size effects in systematic reviews of diagnostic test accuracy was assessed. Journal of Clinical Epidemiology 2005;58(9):882-93.

\section{Ding 2011}

Ding W, Shen Y, Yang J, He X, Zhang M. Diagnosis of pneumothorax by radiography and ultrasonography: a metaanalysis. Chest 2011;140(4):859-66.

\section{Ebrahimi 2014}

Ebrahimi A, Yousefifard M, Mohammad Kazemi H, Rasouli HR, Asady $\mathrm{H}$, Moghadas Jafari A, et al. Diagnostic accuracy of chest ultrasonography versus chest radiography for identification of pneumothorax: a systematic review and meta-analysis. Tanaffos 2014;13(4):29-40.

\section{Fryback 1991}

Fryback DG, Thornbury JR. The efficacy of diagnostic imaging. Medical Decision Making 1991;11(2):88-94.

\section{German Trauma Society 2014}

German Trauma Society (DGU). TraumaRegister DGU. Annual Report 2014. www.dgu-online.de/fileadmin/published content/5.Qualitaet_und_Sicherheit/PDF/2014_TR_DGU_ Jahresbericht.pdf (accessed 14 March 2017).

\section{Holmes 2007}

Holmes JF, Gladman A, Chang CH. Performance of abdominal ultrasonography in pediatric blunt trauma patients: a metaanalysis. Journal of Pediatric Surgery 2007;42(9):1588-94.

\section{Huber-Wagner 2013}

Huber-Wagner S, Biberthaler P, Häberle S, Wierer M, Dobritz M, Rummeny $\mathrm{E}$, et al. Whole-body CT in haemodynamically unstable severely injured patients - a retrospective, multicentre study. PLOS ONE 2013;8(7):e68880.

\section{Lefebvre 2017}

Lefebvre C, Glanville J, Beale S, Boachie C, Duffy S, Fraser C, et al. Assessing the performance of methodological search filters to improve the efficiency of evidence information retrieval: five literature reviews and a qualitative study. Health Technology Assessment 2017;21(69):1-148. [DOI: 10.3310/hta21690]

\section{Lozano 2012}

Lozano R, Naghavi M, Foreman K, Lim S, Shibuya K, Aboyans V, et al. Global and regional mortality from 235 causes of death for 20 age groups in 1990 and 2010: a systematic analysis for the Global Burden of Disease Study 2010. Lancet 2012;380(9859):2095-128.

\section{Macaskill 2011}

Macaskill P, Gatsonis C, Deeks JJ, Harbord RM, Takwoingi Y. Chapter 10: Analysing and presenting results. In: Higgins JP, Green S, editor(s). Cochrane Handbook for Systematic Reviews of Interventions Version 5.1.0 (updated March 2011). The Cochrane Collaboration, 2011. Available from handbook.cochrane.org.

\section{Murray 2012}

Murray CJL, Vos T, Lozano R, Naghavi M, Flaxman AD, Michaud D, et al. Disability-adjusted life years (DALYs) for 291 diseases and injuries in 21 regions, 1990-2010: a systematic analysis for the Global Burden of Disease Study 2010. Lancet 2012;380(9859):2197-223.

\section{Pape 2014}

Pape HC, Lefering R, Butcher N, Peitzman A, Leenen L, Marzi I, et al. The definition of polytrauma revisited: an international consensus process and proposal of the new Berlin definition. Journal of Trauma and Acute Care Surgery 2014;77(5):780-6.

\section{Pfeifer 2016}

Pfeifer R, Teuben M, Andruszkow H, Barkatali BM, Pape HC. Mortality patterns in patients with multiple trauma: a systematic review of autopsy studies. PLOS ONE 2016;11(2):e0148844. 


\section{Poletti 2003}

Poletti PA, Kinkel K, Vermeulen B, Irmay F, Unger PF, Terrier F. Blunt abdominal trauma: should US be used to detect both free fluid and organ injuries?. Radiology 2003;227(1):95-103.

\section{Reitsma 2005}

Reitsma JB, Glas AS, Rutjes AW, Scholten RJ, Bossuyt PM, Zwinderman $\mathrm{AH}$. Bivariate analysis of sensitivity and specificity produces informative summary measures in diagnostic reviews. Journal of Clinical Epidemiology 2005;58(10):982-90.

\section{Review Manager 2014 [Computer program]}

Nordic Cochrane Centre, The Cochrane Collaboration. Review Manager 5 (RevMan 5). Version 5.3. Copenhagen: Nordic Cochrane Centre, The Cochrane Collaboration, 2014.

\section{Scalea 1999}

Scalea T, Rodriguez A, Chiu W, Brenneman F, Fallon W, Kato K, et al. Focused Assessment with Sonography for Trauma (FAST): results from an international consensus conference. Journal of Trauma 1999;46(3):466-72.

\section{Scherer 2007}

Scherer RW, Langenberg P, von Elm E. Full publication of results initially presented in abstracts. Cochrane Database of Systematic Reviews 2007, Issue 2. [DOI: 10.1002/14651858.MR000005.pub3]

\section{Stata 2017 [Computer program]}

StataCorp. Stata Statistical Software: Release 15. Version 15. College Station, TX: StataCorp, 2017.

\section{CHARACTERISTICS OF STUDIES}

Characteristics of included studies [ordered by study ID]

\section{Stengel 2005}

Stengel D, Bauwens K, Rademacher G, Mutze S, Ekkernkamp A. Association between compliance with methodological standards of diagnostic research and reported test accuracy: meta-analysis of focused assessment of US for trauma. Radiology 2005;236(1):102-11.

\section{Stengel 2012}

Stengel D, Ottersbach C, Matthes G, Weigeldt M, Grundei S, Rademacher $\mathrm{G}$, et al. Accuracy of single-pass whole-body computed tomography for detection of injuries in patients with major blunt trauma. CMAJ: Canadian Medical Association Journal 2012;184(8):869-76.

\section{Takwoingi 2016}

Takwoingi Y. Meta-analysis of test accuracy studies in Stata: a bivariate model approach (as supplied April 2016). methods.cochrane.org/sdt/.

\section{Van Vugt 2013}

Van Vugt R, Kool DR, Lubeek SF, Dekker HM, Brink M, Deunk J, et al. An evidence based blunt trauma protocol. Emergency Medicine Journal 2013; Vol. 30(3):e23.

\section{Whiting 2011}

Whiting PF, Rutjes AW, Westwood ME, Mallett S, Deeks JJ, Reitsma JB, et al. QUADAS-2: a revised tool for the quality assessment of diagnostic accuracy studies. Annals of Internal Medicine 2011;155(8):529-36.

\section{Becker 2010}

\section{Study characteristics}

Patient sampling

Patient characteristics and setting
Retrospective study

Study location: Miami, USA

Study period: 2000 to 2005

Care setting: level 1 trauma centre

Mass casualty: no

Participants enrolled: 3181: 2274 men and 907 women

Participants included in analysis: 3181

Age: mean age $39 \pm 19$ years

Type of injury: abdominal trauma

Injury severity: ISS $22.9 \pm 18$

Haemodynamic stability: stable conditions 
Inclusion criteria: blunt trauma patients who underwent both US as a part of initial assessment and CT scan of the abdomen. Patients were divided into 3 groups according to their ISS: Group 1: ISS 1 to 14; Group 2: ISS 16 to 24; Group 3: ISS $\geq 25$ (group allocation is reported as in the published reports - the trialists did not say what happened to patients who had an ISS =15). Age, gender, mechanism of injury, physiologic parameters, laboratory test results, ISS, radiology reports, and all applied procedures were retrospectively reviewed by the study team.

Exclusion criteria: penetrating trauma, no US examination or CT Scan

\section{Index test: FAST}

US protocol: trauma team members performed US examinations on all blunt trauma participants in the resuscitation bay. The US was obtained with the participant in supine position by the attending, trauma fellow, or resident. A positive US examination was considered to be a true positive if the CT scan revealed free fluid, and considered to be a false positive if free fluid was not confirmed at the subsequent CT scan. Negative US findings were counted as true negatives if the CT scan was negative and the participant had an uneventful course, and considered to be a false negative if the participant had a negative US and positive CT examination or was operated on and felt to have a therapeutic laparotomy.

Hardware used: Aloka SSD 1000 (Aloka Co Ltd, Wallingford, CT) with a 3.5$\mathrm{MHz}$ curved probe

Description of imaging technique: 4 areas were examined: perihepatic, perisplenic, pelvic, and pericardial

Target condition and reference standard(s)
Target condition: free fluid

Reference standard: CT (CT technique not specified), laparotomy

Description of technique: not reported

Flow and timing Time between US and reference standard: not reported

Comparative

Notes

\section{Methodological quality}

\begin{tabular}{llll}
\hline Item Authors' judgement & Risk of bias & $\begin{array}{l}\text { Applicability con- } \\
\text { cerns }\end{array}$
\end{tabular}

\section{DOMAIN 1: Patient Selection}

Was a consecutive or random sample of patients en- No rolled?

\begin{tabular}{llll}
\hline Did the study avoid inappropriate exclusions? & Yes & & Low \\
\hline & Unclear & \\
\hline
\end{tabular}

\section{DOMAIN 2: Index Test All tests}

Were the index test results interpreted without knowl- Yes edge of the results of the reference standard? 
Becker 2010 (Continued)

If a threshold was used, was it pre-specified?

Yes

Was the qualification of the US operator appropriate? Unclear

Was the US hardware (i.e. generation, manufacturer, Yes probe, etc.) up to date?

Was the US protocol (i.e. 'classic' FAST) appropriate? Yes

Are there concerns that the definition or performance of the index test (i.e. POC US of trauma) do not match generally accepted, established, or practiced rules or recommendations?

No
Low

Low

\section{DOMAIN 3: Reference Standard}

Is the reference standards likely to correctly classify

Yes

the target condition?

Were the reference standard results interpreted with- Unclear out knowledge of the results of the index tests?

Was the qualification of the doctors (i.e. radiologists, Unclear surgeons, etc.) determining the reference standard appropriate?

Was the reference imaging standard (i.e. MDCT-rows ( 4 to $\geq 256$ slices), contrast-imaging, etc.) up to date?

Are there concerns that the definition or performance of the reference tests (e.g. CT, MRI, laparatomy, thoracotomy, autopsy, etc.) do not match generally accepted, established, or practiced rules or recommendations?

\begin{tabular}{lll}
\hline & & Unclear \\
\hline DOMAIN 4: Flow and Timing & \\
\hline $\begin{array}{l}\text { Was there an appropriate interval between index test } \\
\text { and reference standard? }\end{array}$ & Unclear \\
\hline Did all patients receive the same reference standard? & No \\
\hline Were all patients included in the analysis? & Yes \\
\hline $\begin{array}{l}\text { Did all participants receive a reference standard? } \\
\text { (Risk of partial verification bias) }\end{array}$ & Yes \\
\hline
\end{tabular}

\section{Unclear}


Benya 2000

\section{Study characteristics}

\section{Patient sampling}

Patient characteristics and setting
Prospective study

Study location: Chicago, USA

Study period: October 1996 to October 1997

Care setting: not reported

Mass casualty: no

Participants enrolled: $51: 35$ boys and 16 girls

Participants included in analysis: 51

Age: mean age 6 years 7 months (range 2 weeks to 16 years)

Type of injury: abdominal trauma

Injury severity: not reported

Haemodynamic stability: stable conditions

Inclusion criteria: children with blunt abdominal injuries examined with abdominal CT after initial surgical evaluation were examined with sonography. Only those children who did not require emergency surgery were invited to participate.

Exclusion criteria: not reported
Index tests

\section{Index test: US}

US protocol: abdominal sonography was performed after abdominal $\mathrm{CT}$ in all participants. The paediatric radiologist or sonographer performing the sonography was not aware of the clinical history, physical examination, or CT and laboratory results.

Hardware used: $128 \mathrm{XP} 10$ or Sequoia (Acuson, Mountain View, CA) using a 2.5- to 8-MHz transducer (Acuson), depending on the child's size and physical build

Description of imaging technique: US scan locations: longitudinal and transverse images of both upper quadrants of the abdomen and transverse views of the pancreas, bladder, and both lower quadrants to the abdomen to detect intraperitoneal and retroperitoneal fluid. A variable number of supplemental transverse and longitudinal images of the solid organs in the upper abdomen were subsequently obtained.

\footnotetext{
Target condition and reference standard(s)
}

Reference standard: abdominal and pelvis CT, helically on a HiSpeed Advantage CT scanner (General Electric Medical Systems, Milwaukee, WI)

Description of technique: CT examination during dynamic bolus administration of IV contrast material with slice collimation ranging from $5 \mathrm{~mm}$ to $10 \mathrm{~mm}$, depending on the size of the child 
Benya 2000 (Continued)

Notes

\section{Methodological quality}

\begin{tabular}{llll}
\hline Item Authors' judgement & Risk of bias & $\begin{array}{l}\text { Applicability con- } \\
\text { cerns }\end{array}$
\end{tabular}

\section{DOMAIN 1: Patient Selection}

Was a consecutive or random sample of patients en- Yes rolled?

Did the study avoid inappropriate exclusions? Yes

\section{Low}

Low

\section{DOMAIN 2: Index Test All tests}

Were the index test results interpreted without knowledge Yes of the results of the reference standard?

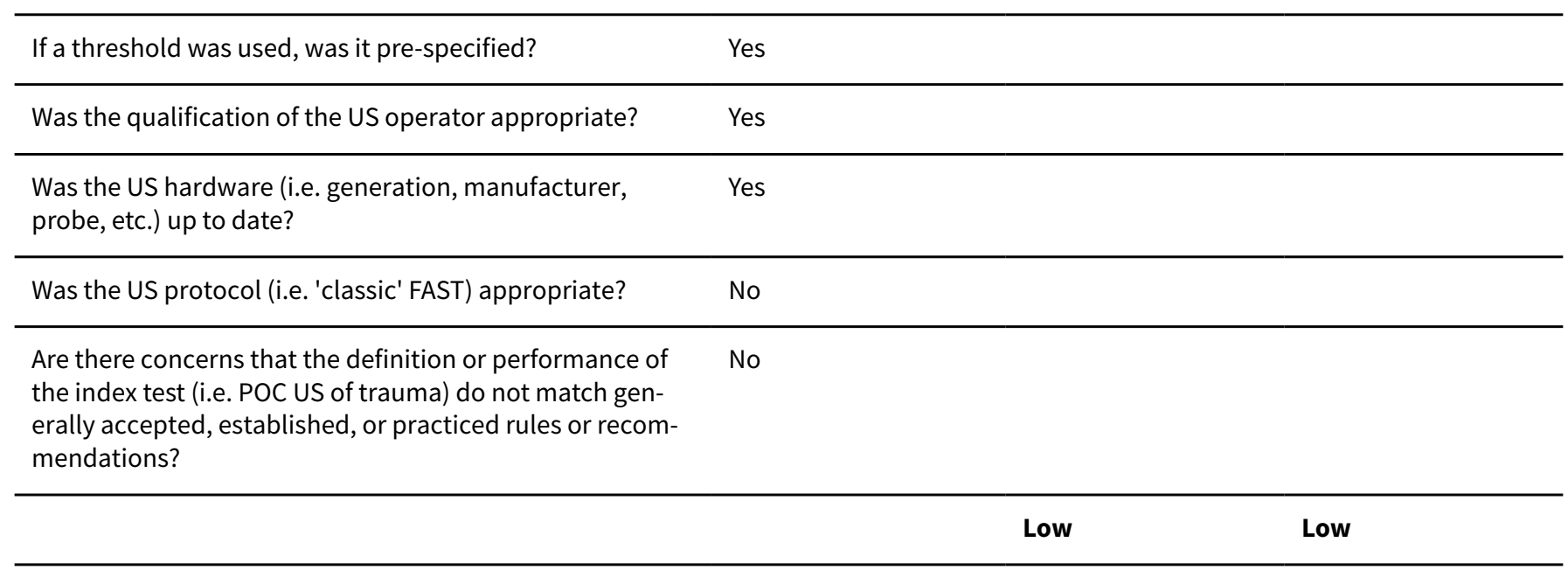

\section{DOMAIN 3: Reference Standard}

Is the reference standards likely to correctly classify the Yes target condition?

Were the reference standard results interpreted without Yes knowledge of the results of the index tests?

Was the qualification of the doctors (i.e. radiologists, sur- Yes geons, etc.) determining the reference standard appropriate?

Was the reference imaging standard (i.e. MDCT-rows (4 to Unclear $\geq 256$ slices), contrast-imaging, etc.) up to date?

Are there concerns that the definition or performance of the reference tests (e.g. CT, MRI, laparatomy, thoracotomy, autopsy, etc.) do not match generally accepted, established, or practiced rules or recommendations? 
Benya 2000 (Continued)

Low

Low

\section{DOMAIN 4: Flow and Timing}

Was there an appropriate interval between index test and No reference standard?

\begin{tabular}{ll}
\hline Did all patients receive the same reference standard? & Yes \\
\hline Were all patients included in the analysis? & Yes \\
\hline
\end{tabular}

Did all participants receive a reference standard? (Risk of Yes

partial verification bias)

Low

\section{Blaivas 2005}

\section{Study characteristics}

Patient sampling

Patient characteristics and setting
Prospective, single-blinded study

Study location: Georgia, USA

Study period: September 2003 to May 2004

Care setting: level 1 trauma centre

Mass casualty: no

Participants enrolled: 176: 100 men and 76 women

Participants included in analysis: 176

Age: $>17$ years

Type of injury: abdominal and chest trauma

Injury severity: not reported

Haemodynamic stability: not reported

Inclusion criteria: blunt trauma patients receiving a FAST examination followed by chest radiography and CT of the chest and/or abdomen and pelvis. Patients who had chest tube placement prior to CT scan were included in the analysis, and the presence of pneumothorax was considered to be verified if a rush of air was heard when the chest tube was inserted.

Exclusion criteria: no completed examination for any reason

\section{Index test: FAST}

US protocol: 4 locations of each hemithorax (anterior second intercostal space at the midclavicular line, fourth intercostal space at the anterior axillary line, sixth intercostal space at the midaxillary line, and sixth intercostal space at the posterior axillary line)

Hardware used: SonoSite 180PLUS (Bothell, WA) using a 4- to 2-MHz microconvex broadband transducer 
Blaivas 2005 (Continued)

Description of imaging technique: US images were obtained parallel to ribs at the rib interspaces. Depth settings were minimised to approximately $5 \mathrm{~cm}$ to optimise magnification of the superficial structures being imaged. Power Doppler was used to enhance the sonologist's ability to identify pleural sliding whenever the sliding lung sign was not easily detected. Absence of the sliding lung sign at the midclavicular point anteriorly or at the fourth interspace at the anterior axillary line was considered indicative of a small pneumothorax; absence of the sliding lung sign at the midaxillary line, a medium pneumothorax; and absence of the sliding lung sign in the posterior axillary line, a large pneumothorax.

Target condition and reference standard(s)

\section{Target condition: free fluid and air}

Reference standard: multigated CT scanner using 5-mm thick slices and portable, supine anteroposterior chest radiographs (technique specification was not reported)

Description of technique: all examinations (US, CT, radiography) were performed with the participant in the supine position
Time between US and reference standard: not reported
Flow and timing

\section{Comparative}

\section{Notes}

\section{Methodological quality}

Item Authors' judgement Risk of bias $\quad$ Applicability concerns

DOMAIN 1: Patient Selection

Was a consecutive or random sample of patients No enrolled?

\begin{tabular}{llll}
\hline Did the study avoid inappropriate exclusions? & Yes & \\
\hline & Unclear & Low \\
\hline
\end{tabular}

\section{DOMAIN 2: Index Test All tests}

Were the index test results interpreted without
knowledge of the results of the reference standard?

\begin{tabular}{ll}
\hline If a threshold was used, was it pre-specified? & Yes \\
\hline $\begin{array}{l}\text { Was the qualification of the US operator appropri- } \\
\text { ate? }\end{array}$ & Yes \\
\hline
\end{tabular}

Was the US hardware (i.e. generation, manufactur- Yes er, probe, etc.) up to date? Was the US protocol (i.e. 'classic' FAST) appropri- Yes
ate? 
Blaivas 2005 (Continued)

not match generally accepted, established, or prac-

ticed rules or recommendations?

\begin{tabular}{lll}
\hline Low & Low \\
\hline
\end{tabular}

\section{DOMAIN 3: Reference Standard}

Is the reference standards likely to correctly classi- $\quad$ Yes

fy the target condition?

Were the reference standard results interpreted Yes

without knowledge of the results of the index tests?

Was the qualification of the doctors (i.e. radiolo- Yes gists, surgeons, etc.) determining the reference standard appropriate?

Was the reference imaging standard (i.e. MD-

CT-rows ( 4 to $\geq 256$ slices), contrast-imaging, etc.)

up to date?

Are there concerns that the definition or perfor-

mance of the reference tests (e.g. CT, MRI, laparato-

my, thoracotomy, autopsy, etc.) do not match gen-

erally accepted, established, or practiced rules or

recommendations?

\begin{tabular}{llll}
\hline LOMAIN 4: Flow and Timing & Low & \\
\hline $\begin{array}{l}\text { Was there an appropriate interval between index } \\
\text { test and reference standard? }\end{array}$ & Yes & \\
\hline $\begin{array}{l}\text { Did all patients receive the same reference stan- } \\
\text { dard? }\end{array}$ & Yes & Yes \\
\hline Were all patients included in the analysis? & Yes & Low \\
\hline $\begin{array}{l}\text { Did all participants receive a reference standard? } \\
\text { (Risk of partial verification bias) }\end{array}$ & \\
\hline
\end{tabular}

Calder 2017

\section{Study characteristics}

Patient sampling Multicentre prospective study

Patient characteristics and setting

Study location: USA

Study period: July 2014 to July 2015

Care setting: 14 paediatric level 1 trauma centre

Mass casualty: no 
Calder 2017 (Continued)

Participants enrolled: 2188: gender not reported

Participants included in analysis: 340

Age: $7.8 \pm 4.6$ years (2188 participants)

Type of injury: abdominal trauma

Injury severity: ISS 5 (IQR 1 to 10 )

Haemodynamic stability: stable and unstable conditions

Inclusion criteria: children $<16$ years of age were enrolled over a 1 -year period. The primary purpose of the data collection was to develop a clinical prediction model to determine which children were at very low risk for intra-abdominal injury following blunt abdominal trauma and could safely avoid abdominal CT as a component of their initial evaluation.

Exclusion criteria: presentation $>6$ hours after injury, abdominal CT imaging prior to arrival at the paediatric trauma centre, isolated head or extremity mechanism of injury, same-level falls, penetrating trauma, burns, and hanging injuries

\section{Index test: FAST}

US protocol: not reported

Hardware used: not reported

Description of imaging technique: not reported

Reference standard: CT (technique specification was not reported) or intraoperative findings, or both

Description of technique: not reported

\section{Comparative}

$$
\text { Notes }
$$

\section{Methodological quality}

\begin{tabular}{|c|c|c|c|}
\hline Item & $\begin{array}{l}\text { Authors' judge- } \\
\text { ment }\end{array}$ & Risk of bias & $\begin{array}{l}\text { Applicability con- } \\
\text { cerns }\end{array}$ \\
\hline
\end{tabular}

\section{DOMAIN 1: Patient Selection}

\begin{tabular}{lll}
\hline Was a consecutive or random sample of patients enrolled? & Unclear \\
\hline Did the study avoid inappropriate exclusions? & Yes & Unclear \\
\hline
\end{tabular}

\section{DOMAIN 2: Index Test All tests}


Calder 2017 (Continued)

Were the index test results interpreted without knowledge of Unclear the results of the reference standard?

\begin{tabular}{lll}
\hline If a threshold was used, was it pre-specified? & Yes & Unclear \\
\hline Was the qualification of the US operator appropriate? & Unclear & \\
\hline $\begin{array}{l}\text { Was the US hardware (i.e. generation, manufacturer, probe, } \\
\text { etc.) up to date? }\end{array}$ & Unclear \\
\hline $\begin{array}{l}\text { Was the US protocol (i.e. 'classic' FAST) appropriate? } \\
\begin{array}{l}\text { Are there concerns that the definition or performance of the in- } \\
\text { dex test (i.e. POC US of trauma) do not match generally accept- } \\
\text { ed, established, or practiced rules or recommendations? }\end{array}\end{array}$ \\
\hline
\end{tabular}

\section{DOMAIN 3: Reference Standard}

Is the reference standards likely to correctly classify the target Yes condition?

Were the reference standard results interpreted without knowl- Unclear edge of the results of the index tests?

Was the qualification of the doctors (i.e. radiologists, surgeons, Unclear etc.) determining the reference standard appropriate?

Was the reference imaging standard (i.e. MDCT-rows (4 to $\geq 256$ Unclear slices), contrast-imaging, etc.) up to date?

Are there concerns that the definition or performance of the ref- Unclear erence tests (e.g. CT, MRI, laparatomy, thoracotomy, autopsy, etc.) do not match generally accepted, established, or practiced rules or recommendations?

\begin{tabular}{lll}
\hline & Unclear & Unclear \\
\hline DOMAIN 4: Flow and Timing & &
\end{tabular}

Was there an appropriate interval between index test and refer- Yes ence standard?

\begin{tabular}{lc} 
Did all patients receive the same reference standard? & No \\
\hline Were all patients included in the analysis? & Yes \\
\hline $\begin{array}{l}\text { Did all participants receive a reference standard? (Risk of par- } \\
\text { tial verification bias) }\end{array}$ & Yes
\end{tabular}

\section{No}

Yes

es 
Catalano 2009

\section{Study characteristics}

\begin{tabular}{ll}
\hline Patient sampling & Multicentre prospective study \\
\hline Patient characteristics and set- & Study location: Italy and the UK \\
ting & Study period: not reported \\
& Care setting: 6 centres (type of trauma centre not reported)
\end{tabular}

Mass casualty: no

Participants enrolled: 156: 118 men and 38 women

Participants included in analysis: 156

Age: mean age $39 \pm 17$ years (range 15 to 90 years)

Type of injury: solid organ injury after blunt abdominal trauma

Injury severity: ISS range 0 to 50

Haemodynamic stability: stable and unstable conditions

Inclusion criteria: > 14 years of age, clinical and laboratory suspicion of blunt or penetrating abdominal trauma, availability of US, CEUS, and CT study performed within 1 hour, availability of a reference standard (CT or surgery)

Exclusion criteria: not reported

Index tests

\section{Index test: US, CEUS}

US protocol: 4 locations of each hemithorax (anterior second intercostal space at the midclavicular line, fourth intercostal space at the anterior axillary line, sixth intercostal space at the midaxillary line, and sixth intercostal space at the posterior axillary line)

Hardware used: US with an EsaTune (Esaote, Italy), Technos (Esaote, Italy), or ATL HDI 5000 (Philips, the Netherlands), phased-array transducers (2 to $6 \mathrm{MHz}$ ). In selected cases, the operator also employed high-frequency probes (5.5 to $10 \mathrm{MHz}$ ), power Doppler imaging mode, and/or tissue harmonic imaging mode to maximise diagnostic effectiveness of unenhanced US. CEUS with a 2.5- or 3.5-MHz transducer and a low acoustic power setting (mechanical index 0.05 to 0.1 ) - 'second-generation' contrast medium SonoVue (Bracco, Milan, Italy)

Description of imaging technique: in all cases, a rapid but complete survey of all abdominal parenchymas and abdomino-pelvic spaces was obtained by using conventional grey-scale US imaging. A careful search for peritoneal fluid, retroperitoneal fluid, and organ injury was carried out. CEUS studies were carried out with the harmonic, low mechanical index, contrast-specific softwares contrast tuned imaging ( $\mathrm{CnTI}$ ) and pulse inversion. CEUS was always performed immediately after the baseline US. SonoVue volume $(4.8 \mathrm{~mL})$ was fractionated into $2 \times 2.4 \mathrm{~mL}$ doses, each injected as a quick bolus through an antecubital vein and a 18- to 20-gauge catheter followed by $5 \mathrm{~mL}$ to $10 \mathrm{~mL}$ normal saline $(0.9 \% \mathrm{NaCl})$ flush through a 3-way stopcock. Immediately after the first contrast medium injection, the right-sided organs (the right kidney, and possibly adrenal first and the liver subsequently) were explored for 1 to 3 minutes. Thereafter, the second SonoVue dose was administered, focusing on left-side organs (the left kidney, and possibly adrenal first, the pancreas, and finally the spleen) for another 3 to 4 minutes.

\section{Target condition: free fluid and air}

Reference standard: CT and/or surgery, CT Somatom Plus 4 system (Siemens AG, Germany), Emotion system (Siemens), PQ6000 system (Picker International, USA), LightSpeed Ultra system (GE Healthcare, USA), and Sensation 16-row tomograph (Siemens) 
Description of technique: no participant received oral contrast medium, and most had a precontrast acquisition series. The contrast-enhanced study was carried out using 5- or 8-mm collimation, 5- or 7.5-mm/s table speed, $120 \mathrm{kVp}, 5-\mathrm{mm}$ reconstruction interval. A non-ionic contrast medium (a combination of iomeprol $350 \mathrm{mgl} / \mathrm{mL}$, Iomeron, Bracco-iopamidol $300 \mathrm{mgl} / \mathrm{mL}$, Iopamiro, Bracco-iohexol $350 \mathrm{mgl} / \mathrm{mL}$, Omnipaque, produced by Amersham Health) was administered via 18- to 20-gauge angiocatheter and power injector. A volume of $100 \mathrm{~mL}$ to $150 \mathrm{~mL}$ was injected at $2 \mathrm{~mL} / \mathrm{s}$ to $4 \mathrm{~mL} / \mathrm{s}$. When a 2-phase technique was employed, delay ranged from $40 \mathrm{~s}$ to $50 \mathrm{~s}$ for the first contrast-enhanced acquisition and from $80 \mathrm{~s}$ to $120 \mathrm{~s}$ for the second acquisition. Participants undergoing single-phase acquisition were scanned at $60 \mathrm{~s}$ to $90 \mathrm{~s}$ from contrast injection $(2 \mathrm{~mL} / \mathrm{s})$. A true positive was defined as the presence of an abdominal lesion based on both US and the truth standard. A true negative was defined as the absence of an abdominal lesion based on both US and the truth standard.

Flow and timing Time between US and reference standard: $<60$ minutes

\section{Comparative}

Notes

\section{Methodological quality}

\begin{tabular}{llll}
\hline Item & Authors' judgement & Risk of bias & Applicability concerns
\end{tabular}

\section{DOMAIN 1: Patient Selection}

Was a consecutive or random Unclear

sample of patients enrolled?

Did the study avoid inappropriate Yes exclusions?

\begin{tabular}{lcc}
\hline & Unclear & Low \\
\hline DOMAIN 2: Index Test All tests &
\end{tabular}

\section{DOMAIN 2: Index Test All tests}

Were the index test results inter- 
Catalano 2009 (Continued)

\title{
DOMAIN 3: Reference Standard
}

Is the reference standards like-

ly to correctly classify the target condition?

\author{
Were the reference standard re- \\ sults interpreted without knowl- \\ edge of the results of the index \\ tests?
}

Was the qualification of the doctors (i.e. radiologists, surgeons, etc.) determining the reference standard appropriate?

Was the reference imaging standard (i.e. MDCT-rows ( 4 to $\geq 256$

slices), contrast-imaging, etc.) up to date?

Are there concerns that the definition or performance of the reference tests (e.g. CT, MRI, laparatomy, thoracotomy, autopsy, etc.) do not match generally accepted, established, or practiced rules or recommendations?

\section{Low}

\section{DOMAIN 4: Flow and Timing}

Was there an appropriate interval Yes between index test and reference standard?

Did all patients receive the same No reference standard?

\section{Were all patients included in the Yes} analysis?

Did all participants receive a ref- Yes erence standard? (Risk of partial verification bias)

\section{Cheung 2012}

\section{Study characteristics}


Cheung 2012 (Continued)

Patient sampling

Patient characteristics and setting
Retrospective study

Study location: Hong Kong, China

Study period: January 2005 to December 2010

Care setting: emergency department (type of trauma centre not reported)

Mass casualty: no

Participants enrolled: 302

Participants included in analysis: $156: 103$ men and 50 women

Age: mean age 48.6 years (range 3 to 94 years)

Type of injury: blunt abdominal trauma

Injury severity: not reported

Haemodynamic stability: stable and unstable conditions

Inclusion criteria: all the blunt abdominal trauma patients identified in the hospital trauma registry and managed by the hospital trauma team in the emergency department

Exclusion criteria: death at the emergency department after initial resuscitation; known ascites or peritoneal dialysis before the injury; blunt abdominal trauma patients who did not undergo FAST or standard confirmatory test; those with incomplete FAST findings

\section{Index test: FAST}

US protocol: FAST procedures followed the standard recommendation. Scanning was done on supine participants at 6 sites.

Hardware used: ALOKASSD-500 (probe model UST-934N-3.5, 3.5-MHz convex sector probe) and GE Medical Logiq P5 Premium Ultrasound Console (probe model 4C 1.8-4.0/D2.9MHz, curvilinear probe)

Description of imaging technique: during the study period, there was no formal guideline in the hospital on how and when to perform the FAST. However, it was usual practice for FAST to be done by the emergency physicians or surgeons in charge of the trauma team in the emergency department, who had to be certified advanced trauma life support providers. 6 views were evaluated: the 4 quadrants of the abdomen and the suprapubic and subxiphoid region.

Target condition: free fluid and air

Reference standard: laparotomy, CT (technical specification was not reported), autopsy

Description of technique: laparotomy started within 4 hours of admission was used as a gold standard confirmatory test for presence of intraperitoneal bleeding in blunt abdominal trauma participants for whom surgical exploration was clinically indicated. If the participant did not require laparotomy, abdominal CT scan was taken as the gold standard when the participant was still treated in the emergency department. Autopsy, in addition to CT scan if available, was used as surrogate standard test for participants who died during hospital stay without laparotomy performed. 
Cheung 2012 (Continued)

Comparative

\section{Notes}

\section{Methodological quality}

\begin{tabular}{llll}
\hline Item Authors' judgement & Risk of bias & Applicability concerns
\end{tabular}

DOMAIN 1: Patient Selection

Was a consecutive or random sample of patients No enrolled?

Did the study avoid inappropriate exclusions? Yes

\begin{tabular}{lll}
\hline Unclear & Low \\
\hline
\end{tabular}

\section{DOMAIN 2: Index Test All tests}

Were the index test results interpreted without Unclear knowledge of the results of the reference standard?

If a threshold was used, was it pre-specified? Yes

Was the qualification of the US operator appropri- Yes ate?

Was the US hardware (i.e. generation, manufac- Yes turer, probe, etc.) up to date?

Was the US protocol (i.e. 'classic' FAST) appropri- No ate?

Are there concerns that the definition or performance of the index test (i.e. POC US of trauma) do not match generally accepted, established, or practiced rules or recommendations?

\begin{tabular}{|c|c|c|}
\hline & Unclear & Low \\
\hline \multicolumn{3}{|l|}{ DOMAIN 3: Reference Standard } \\
\hline $\begin{array}{l}\text { Is the reference standards likely to correctly clas- } \\
\text { sify the target condition? }\end{array}$ & Yes & \\
\hline $\begin{array}{l}\text { Were the reference standard results interpret- } \\
\text { ed without knowledge of the results of the index } \\
\text { tests? }\end{array}$ & Unclear & \\
\hline $\begin{array}{l}\text { Was the qualification of the doctors (i.e. radiolo- } \\
\text { gists, surgeons, etc.) determining the reference } \\
\text { standard appropriate? }\end{array}$ & Unclear & \\
\hline $\begin{array}{l}\text { Was the reference imaging standard (i.e. MD- } \\
\text { CT-rows ( } 4 \text { to } \geq 256 \text { slices), contrast-imaging, etc.) } \\
\text { up to date? }\end{array}$ & Unclear & \\
\hline
\end{tabular}


Cheung 2012 (Continued)

Are there concerns that the definition or performance of the reference tests (e.g. CT, MRI, laparatomy, thoracotomy, autopsy, etc.) do not match generally accepted, established, or practiced rules or recommendations?

Unclear

High

\section{DOMAIN 4: Flow and Timing}

Was there an appropriate interval between index Yes test and reference standard?

Did all patients receive the same reference stan- No dard?

\begin{tabular}{ll}
\hline Were all patients included in the analysis? & Yes \\
\hline $\begin{array}{l}\text { Did all participants receive a reference standard? } \\
\text { (Risk of partial verification bias) }\end{array}$ \\
\hline
\end{tabular}

Low

\section{Clevert 2008}

\section{Study characteristics}

\begin{tabular}{|c|c|}
\hline Patient sampling & Prospective study \\
\hline \multirow[t]{12}{*}{ Patient characteristics and setting } & Study location: Germany \\
\hline & Study period: January 2005 to January 2007 \\
\hline & Care setting: not reported \\
\hline & Mass casualty: no \\
\hline & Participants enrolled: $78: 48$ men and 30 women \\
\hline & Participants included in analysis: 78 \\
\hline & Age: mean age 56 years \\
\hline & Type of injury: blunt abdominal trauma \\
\hline & Injury severity: not reported \\
\hline & Haemodynamic stability: not reported \\
\hline & $\begin{array}{l}\text { Inclusion criteria: all blunt abdominal trauma patients who were examined by convention- } \\
\text { al US, CEUS, and MSCT }\end{array}$ \\
\hline & Exclusion criteria: not reported \\
\hline \multirow[t]{2}{*}{ Index tests } & Index test: US, CEUS, and MSCT \\
\hline & $\begin{array}{l}\text { US protocol: a conventional B-scan followed by colour-coded duplex sonography. The } \\
\text { colour gain was set just high enough to avoid overwriting artefacts (i.e. colour pixels outside }\end{array}$ \\
\hline
\end{tabular}


Clevert 2008 (Continued)

the perfused lumen of the vessel). Additionally, an automatic image gain optimisation could be selected.

Hardware used: Siemens ACUSON Sequoia 512 (Siemens Medical Systems, Forchheim, Germany) using a curved array 4-MHz multifrequency transducer

Description of imaging technique: for CEUS examinations the Sequoia system was equipped with contrast pulse sequence software that detects the microbubbles' fundamental non-linear response. Depending on participant size and the target organ, a bolus intravenous injection of $1.2 \mathrm{~mL}$ to $2.4 \mathrm{~mL}$ of a second-generation blood pool contrast agent (SonoVue, Bracco, Milan, Italy) consisting of stabilised microbubbles of sulphur hexafluoride was administered into an antecubital vein through an 18-gauge needle and was followed by a flush of $10 \mathrm{~mL}$ saline solution $(0.9 \% \mathrm{NaCl})$. CEUS employed continuous low mechanical index ( 0.15 to 0.19 ) real-time tissue harmonic imaging (Cadence) contrast pulse sequence imaging. Sonography and contrast-enhanced sonography findings were considered true positives if $\mathrm{CT}$ revealed evidence of the parenchyma injury, and false positives if the injury was not confirmed on subsequent CT. Negative sonography findings were declared as true negatives if $\mathrm{CT}$ findings were negative and the participant had an uneventful clinical course; findings were declared as false negatives if CT revealed parenchyma injury. A solid organ injury on sonography was reported if an intraparenchymal hyper- or hypoechoic area or a distortion of the normal echoic structure was seen.

Target condition and reference stan$\operatorname{dard}(\mathrm{s})$

\section{Target condition: organ injuries/vascular lesions}

Reference standard: CECT, Scanner Somaton Sensation 16 or 64, Siemens Medical Systems, Forchheim, Germany

Description of technique: the entire study population underwent contrast-enhanced CT examinations using a standard arterial and venous phase protocol with a 16- or 64-detector CT scanner. For the Sensation 64 , collimation and table feed were $64 \times 0.6 \mathrm{~mm}$, rotation time $0.33 \mathrm{~s}$, pitch 0.9 , slice thickness $0.75 \mathrm{~mm}$, and reconstruction interval $0.5 \mathrm{~mm}$. Tube voltage was set to $120 \mathrm{kV}$, and the exposure time $\times$ tube current product was $200 \mathrm{mAs}$ using Care Dose 4-D. For the Sensation 16, collimation and table feed were $16 \times 0.75 \mathrm{~mm}$, rotation time $0.5 \mathrm{~s}$, pitch 1 , slice thickness $0.75 \mathrm{~mm}$, and reconstruction interval $0.6 \mathrm{~mm}$. Tube voltage was set to $100 \mathrm{kV}$, and the exposure time $\times$ tube current product was $220 \mathrm{mAs}$ using Care Dose 4-D. The contrast agent was injected into an antecubital vein as a bolus, using a dual-head power injector with a flow rate of $5 \mathrm{~mL} / \mathrm{s} .120 \mathrm{~mL}$ of Solutrast (Bracco, Milan, Italy) with an iodine concentration of $300 \mathrm{mg} / \mathrm{mL}$ was administered, followed by $50 \mathrm{~mL}$ saline. The appropriate scan delay for the arterial and venous phase after contrast agent administration was determined by semiautomatic bolus tracking on the thoracic aorta.

Comparative

$$
\text { Notes }
$$

Methodological quality

\begin{tabular}{llll}
\hline Item & Authors' judgement & Risk of bias & Applicability concerns \\
\hline
\end{tabular}

DOMAIN 1: Patient Selection

Was a consecutive or random sample No

of patients enrolled?

Did the study avoid inappropriate ex- Yes clusions?

\section{Unclear}

Low

Point-of-care ultrasonography for diagnosing thoracoabdominal injuries in patients with blunt trauma (Review)

Copyright $\odot 2018$ The Cochrane Collaboration. Published by John Wiley \& Sons, Ltd. 
Clevert 2008 (Continued)

\section{DOMAIN 2: Index Test All tests}

Were the index test results interpreted without knowledge of the results of the reference standard?

\section{If a threshold was used, was it pre- Yes specified?}

Was the qualification of the US opera- Yes tor appropriate?

\section{Was the US hardware (i.e. genera- Yes \\ tion, manufacturer, probe, etc.) up to}

date?

Was the US protocol (i.e. 'classic' Unclear
FAST) appropriate?

FAST) appropriate?

\section{Are there concerns that the definition Unclear} or performance of the index test (i.e. POC US of trauma) do not match generally accepted, established, or practiced rules or recommendations?

\begin{tabular}{lll}
\hline & Low & Unclear \\
\hline
\end{tabular}

\section{DOMAIN 3: Reference Standard}

\section{Is the reference standards likely to Yes} correctly classify the target condition?

\section{Were the reference standard results} interpreted without knowledge of the results of the index tests?

Was the qualification of the doctors (i.e. radiologists, surgeons, etc.) determining the reference standard appropriate?

\section{Was the reference imaging standard Yes} (i.e. MDCT-rows ( 4 to $\geq 256$ slices), contrast-imaging, etc.) up to date?

Are there concerns that the definition or performance of the reference tests (e.g. CT, MRI, laparatomy, thoracotomy, autopsy, etc.) do not match generally accepted, established, or practiced rules or recommendations?

\begin{tabular}{lll}
\hline & Low & Low \\
\hline DOMAIN 4: Flow and Timing &
\end{tabular}




\section{Clevert 2008 (Continued)}

Was there an appropriate interval be- Yes tween index test and reference standard?

Did all patients receive the same ref- Yes erence standard?

Were all patients included in the Yes analysis?

Did all participants receive a referYes ence standard? (Risk of partial verification bias)

Low

Coley 2000

\section{Study characteristics}

Patient sampling

Patient characteristics and setting
Prospective study

Study location: USA

Study period: July 1997 to August 1998

Care setting: level I paediatric trauma centre

Mass casualty: no

Participants enrolled: $107: 68$ boys and 38 girls

Participants included in analysis: 97

Age: mean age 95 months \pm 51 months (range 2 to 216 months)

Type of injury: blunt abdominal trauma

Injury severity: GCS $12.5 \pm 4.2$, PTS $8.0 \pm 2.8$

Haemodynamic stability: stable conditions

Inclusion criteria: all children suffering blunt abdominal trauma who required a CT scan for evaluation of potential injury based on the surgical team leader's clinical judgement

Exclusion criteria: haemodynamically unstable, and required immediate operative intervention

\section{Index test: FAST}

US protocol: immediately before CT scan, the in-house on-call paediatric radiologist performed the FAST scan and rendered a decision regarding the absence or presence and location of any free fluid. If participants had a Foley catheter placed, this was clamped as soon as possible to allow the bladder to fill, facilitating detection of free fluid in the pelvis.

Hardware used: Acuson 1283 P10 system (Mountain View, CA) by using 2- to 5-MHz transducers, depending on participant body habitus 
Coley 2000 (Continued)

Target condition and reference standard(s)

Description of imaging technique: 4 specified locations were evaluated: the right upper quadrant, left upper quadrant, subxyphoid region, and pelvis

Target condition: free fluid and air

Reference standard: CECT, third-generation spiral scanner (High Speed Advantage, General Electric, Milwaukee, WI) with IV but without oral contrast (more technical specifications were not reported)

Description of technique: CT scans were evaluated for the absence or presence of fluid, its location, and the presence of any visceral injury

Flow and timing

Time between US and reference standard: not reported

Comparative

Notes

\section{Methodological quality}

\begin{tabular}{llll}
\hline Item Authors' judgement & Risk of bias & $\begin{array}{l}\text { Applicability con- } \\
\text { cerns }\end{array}$
\end{tabular}

\section{DOMAIN 1: Patient Selection}

\begin{tabular}{lll}
\hline Was a consecutive or random sample of patients enrolled? & Yes \\
\hline Did the study avoid inappropriate exclusions? & Yes & Low \\
\hline
\end{tabular}

\section{DOMAIN 2: Index Test All tests}

Were the index test results interpreted without knowledge Unclear of the results of the reference standard?

\begin{tabular}{ll}
\hline If a threshold was used, was it pre-specified? & Yes \\
\hline Was the qualification of the US operator appropriate? & Yes
\end{tabular}

Was the US hardware (i.e. generation, manufacturer, probe, Yes etc.) up to date?

\begin{tabular}{l} 
Was the US protocol (i.e. 'classic' FAST) appropriate? \\
\hline Are there concerns that the definition or performance of \\
the index test (i.e. POC US of trauma) do not match gener- \\
ally accepted, established, or practiced rules or recommen- \\
dations?
\end{tabular}

\begin{tabular}{lll}
\hline & Low & Low \\
\hline DOMAIN 3: Reference Standard & &
\end{tabular}

Is the reference standards likely to correctly classify the tar- Yes get condition? 
Coley 2000 (Continued)

Were the reference standard results interpreted without Yes knowledge of the results of the index tests?

Was the qualification of the doctors (i.e. radiologists, surgeons, etc.) determining the reference standard appropriate?

Was the reference imaging standard (i.e. MDCT-rows (4 to $\geq \quad$ Unclear

256 slices), contrast-imaging, etc.) up to date?

Are there concerns that the definition or performance of

No

the reference tests (e.g. CT, MRI, laparatomy, thoracoto-

my, autopsy, etc.) do not match generally accepted, estab-

lished, or practiced rules or recommendations?

Low

Low

\section{DOMAIN 4: Flow and Timing}

Was there an appropriate interval between index test and Yes reference standard?

\begin{tabular}{ll}
\hline Did all patients receive the same reference standard? & Yes \\
\hline Were all patients included in the analysis? & Yes \\
\hline $\begin{array}{l}\text { Did all participants receive a reference standard? (Risk of } \\
\text { partial verification bias) }\end{array}$ & Yes \\
\hline
\end{tabular}

Low

\section{Corbett 2000}

\section{Study characteristics}

Patient sampling Prospective study

\section{Patient characteristics and setting}

Study location: California, USA

Study period: June 1995 to February 1996

Care setting: level I paediatric trauma centre

Mass casualty: no

Participants enrolled: 81: gender specification not reported

Participants included in analysis: 47

Age: mean age 9 years (range 2 to 7 years)

Type of injury: abdominal and pelvic trauma

Injury severity: ISS 9.0 (IQR 2.5 to 13 )

Haemodynamic stability: not reported 
Inclusion criteria: all children for whom paediatric trauma service consultation was requested and for whom a designated ultrasonographer was available were included. Trauma service consultation was requested on those with moderate to severe injuries judged by the emergency physician to require additional evaluation.

Exclusion criteria: conversely, patients $\geq 18$ years, those receiving minor trauma that was not felt to require trauma service evaluation, and those for whom a designated ultrasonographer was not available

\section{Index test: US}

US protocol: consecutive children requiring further emergency department evaluation by the paediatric trauma service received a rapid emergency department US examination during their initial resuscitation. US examinations were performed by 17 different emergency medicine residents and attending physicians.

Hardware used: Scanner 200 (Pie Medical, Boca Raton, FL) using a 3.5 or 5.0 curvilinear probe

Description of imaging technique: 7 views were examined: subxiphoid, right upper quadrant oblique and coronal, left upper quadrant oblique and coronal, pelvic transverse and pelvic longitudinal

Target condition and reference standard(s)

\section{Target condition: free fluid and air}

Reference standard: CT (technique specification not reported), laparotomy

Description of technique: the decision to take a participant for laparotomy or to the CT scanner was made by the trauma team captain based on the usual clinical, laboratory, and radiographic (but not US) findings. CT scans were considered positive if the presence of free fluid was recorded in the radiologists' interpretation. Laparotomies were considered positive if free fluid was noted in the operative note. Other surgical or diagnostic procedures capable of showing the presence of free fluid were also reviewed.

Flow and timing

Time between US and reference standard: not reported

Comparative

$$
\text { Notes }
$$

Methodological quality

\begin{tabular}{llll}
\hline Item & Authors' judgement & Risk of bias & Applicability concerns \\
\hline DOMAIN 1: Patient Selection & & \\
\hline $\begin{array}{l}\text { Was a consecutive or random sample of patients } \\
\text { enrolled? }\end{array}$ & No & & High \\
\hline Did the study avoid inappropriate exclusions? & Yes & Unclear & \\
\hline
\end{tabular}

\section{DOMAIN 2: Index Test All tests}

Were the index test results interpreted without

Yes

knowledge of the results of the reference standard? 
Corbett 2000 (Continued)

If a threshold was used, was it pre-specified? Yes

Was the qualification of the US operator appropri- No ate?

Was the US hardware (i.e. generation, manufactur- Yes er, probe, etc.) up to date?

Was the US protocol (i.e. 'classic' FAST) appropri- No ate?

Are there concerns that the definition or perforNo mance of the index test (i.e. POC US of trauma) do not match generally accepted, established, or practiced rules or recommendations?

\begin{tabular}{lll}
\hline Unclear & Low \\
\hline
\end{tabular}

\section{DOMAIN 3: Reference Standard}

Is the reference standards likely to correctly classi- Yes fy the target condition?

Were the reference standard results interpreted Yes without knowledge of the results of the index tests?

\section{Was the qualification of the doctors (i.e. radiolo- Yes} gists, surgeons, etc.) determining the reference standard appropriate?

\section{Was the reference imaging standard (i.e. MD- \\ CT-rows ( 4 to $\geq 256$ slices), contrast-imaging, etc.) up to date?}

Are there concerns that the definition or performance of the reference tests (e.g. CT, MRI, laparatomy, thoracotomy, autopsy, etc.) do not match generally accepted, established, or practiced rules or recommendations?

\begin{tabular}{ll}
\hline LOMAIN 4: Flow and Timing & Low \\
\hline Was there an appropriate interval between index & Yes \\
test and reference standard? & Yes \\
\hline $\begin{array}{l}\text { Did all patients receive the same reference stan- } \\
\text { dard? }\end{array}$ & Yes \\
\hline $\begin{array}{l}\text { Were all patients included in the analysis? } \\
\text { Did all participants receive a reference standard? }\end{array}$ & Yes \\
\hline Risk of partial verification bias)
\end{tabular}


Dolich 2001

\section{Study characteristics}

\begin{tabular}{ll}
\hline Patient sampling & Retrospective study \\
\hline
\end{tabular}

Patient characteristics and setting

\section{Study location: USA}

Study period: 30-month period ending July 1997

Care setting: level I trauma centre

\section{Mass casualty: no}

Participants enrolled: 2576: 1880 men and 696 women

Participants included in analysis: 616

Age: mean age 38 years (range 1 to 94 years)

Type of injury: blunt abdominal trauma

Injury severity: GCS $\leq 12$

Haemodynamic stability: stable and unstable condition

Inclusion criteria: all patients had abdominal US in the evaluation of blunt abdominal trauma and were entered into a trauma US database. This database was analysed to determine the utility of US in the evaluation of blunt abdominal trauma.

Exclusion criteria: not reported

\section{Index test: US}

US protocol: real-time US images were interpreted by an attending radiologist or senior radiology resident in conjunction with the trauma surgery attending or fellow, or both. The evidence of free intraperitoneal fluid or parenchymal injury was considered a positive result. US was considered negative if the above were absent on a technically satisfactory examination. US examinations were deemed to be indeterminate if they revealed questionable free fluid or solid organ injury, or were technically limited.

Hardware used: Accuson $128 \mathrm{X}$ P/10 (Mountain View, CA) with a 3.5-MHz sector transducer

Description of imaging technique: with the participant in the supine position, views of the pericardium, bilateral subphrenic spaces, Morison's pouch, perisplenic region, and pelvis were examined for the presence of free intraperitoneal fluid or solid organ injury

\footnotetext{
Target condition and reference standard(s)
}

\section{Target condition: free fluid, solid organ injury}

Reference standard: CT (technical specifications not reported), exploratory laparotomy (DPL, observation)

Description of technique: in general, unstable participants with a positive US were taken to the operating room for exploratory laparotomy. Participants with a positive US who remained haemodynamically stable during the initial assessment in the resuscitation area underwent abdominal CT scan to evaluate further the extent and nature of intra-abdominal injury. For statistical analysis, a true-positive result was defined as a positive US with confirmation of injury by CT scan, DPL, or exploratory laparotomy. A negative US with confirmation by observation, CT scan, DPL, or laparotomy was deemed a true negative. A false-positive result was defined as a pos- 
Dolich 2001 (Continued)

itive US with subsequent absence of intra-abdominal injury by CT scan, DPL, or laparotomy. A false-negative result was defined as a negative US in a participant with intra-abdominal injury, as documented by CT scan, DPL, or exploratory laparotomy.

Flow and timing

Time between US and reference standard: not reported

Comparative

Notes

\section{Methodological quality}

\begin{tabular}{llll}
\hline Item & Authors' judgement & Risk of bias & Applicability concerns \\
\hline DOMAIN 1: Patient Selection & & \\
\hline $\begin{array}{l}\text { Was a consecutive or random sample of pa- } \\
\text { tients enrolled? }\end{array}$ & Unclear & \\
\hline Did the study avoid inappropriate exclusions? & Yes & Unclear & Low \\
\hline
\end{tabular}

\section{DOMAIN 2: Index Test All tests}

\section{Were the index test results interpreted with- Unclear} out knowledge of the results of the reference standard?

\begin{tabular}{ll}
\hline If a threshold was used, was it pre-specified? & Yes \\
\hline $\begin{array}{ll}\text { Was the qualification of the US operator ap- } & \text { Yes } \\
\text { propriate? } & \end{array}$
\end{tabular}

Was the US hardware (i.e. generation, manu- Yes

facturer, probe, etc.) up to date?
Was the US protocol (i.e. 'classic' FAST) ap- No propriate?

Are there concerns that the definition or performance of the index test (i.e. POC US of trauma) do not match generally accepted, established, or practiced rules or recommendations?

\begin{tabular}{l}
\hline Unclear \\
\hline DOMAIN 3: Reference Standard \\
\hline $\begin{array}{l}\text { Is the reference standards likely to correctly Unclear } \\
\text { classify the target condition? }\end{array}$ \\
$\begin{array}{l}\text { Were the reference standard results interpret- } \\
\text { ed without knowledge of the results of the in- } \\
\text { dex tests? }\end{array}$ \\
\hline
\end{tabular}


Dolich 2001 (Continued)

Was the qualification of the doctors (i.e. radi- Unclear ologists, surgeons, etc.) determining the reference standard appropriate?

Was the reference imaging standard (i.e. MDCT-rows ( 4 to $\geq 256$ slices), contrast-imaging, etc.) up to date?

Are there concerns that the definition or performance of the reference tests (e.g. CT, MRI, laparatomy, thoracotomy, autopsy, etc.) do not match generally accepted, established, or practiced rules or recommendations?

\begin{tabular}{llll}
\hline & Unclear & High \\
\hline
\end{tabular}

\section{DOMAIN 4: Flow and Timing}

Was there an appropriate interval between in- Yes dex test and reference standard?

Did all patients receive the same reference No standard?

Were all patients included in the analysis? Yes

Did all participants receive a reference stan- Yes

dard? (Risk of partial verification bias)

\section{Low}

\section{Emery 2001}

\section{Study characteristics}

Patient sampling Prospective study

Patient characteristics and setting

Study location: California, USA

Study period: 1 February 1997 to 30 June 1998

Care setting: level I paediatric trauma centre

Mass casualty: no

Participants enrolled: 491

Participants included in analysis: 160: 95 boys and 65 girls

Age: mean age 9 years 5 months (range 1 month to 18 years)

Type of injury: blunt abdominal trauma

Injury severity: ISS 5 (IQR 1 to 13 )

Haemodynamic stability: stable conditions 
Inclusion criteria: all haemodynamically stable paediatric trauma victims referred for abdominal CT initially underwent rapid screening sonography looking for free fluid

Exclusion criteria: not reported

$\begin{array}{ll}\text { Index tests } & \text { Index test: FAST }\end{array}$

US protocol: scans were usually done immediately before the CT scan (<15 minutes)

Hardware used: Acuson unit (Mountain View, CA), 3- to 5-MHz sector or curved transducer

Description of imaging technique: locations: each of the 4 abdominal quadrants and the pelvis ( 2 longitudinal and transverse images in each of the upper quadrants and 1 longitudinal and transverse image in each of the lower quadrants and midline pelvis). No specific epigastric or pericardial view was obtained. The study was focused to identify free fluid. The amount of fluid (none, trace, small, moderate, or large), location(s), and the degree of bladder filling (empty, partially filled, or full) were recorded.

Target condition and reference standard(s)

Target condition: free fluid and air

Reference standard: GE HiSpeed Advantage helical CECT (General Electric Medical Systems, Milwaukee, WI) using non-ionic IV contrast (Optiray 320 (Mallinckrodt, St Louis, MO))

Description of technique: all CT scans included the abdomen and pelvis using a CT and non-ionic IV contrast in a standard dose of $2 \mathrm{~mL} /$ $\mathrm{kg}$ administered in bolus fashion. Slice collimation was either $7 \mathrm{~mm}$ or $10 \mathrm{~mm}$ depending on the participant's size with a pitch of 1.3:1.

Flow and timing

Time between US and reference standard: $<15$ minutes

Comparative

$$
\text { Notes }
$$

Methodological quality

\begin{tabular}{llll}
\hline Item & Authors' judgement & Risk of bias & $\begin{array}{l}\text { Applicability con- } \\
\text { cerns }\end{array}$ \\
\hline
\end{tabular}

\section{DOMAIN 1: Patient Selection}

Was a consecutive or random sample of patients en-

rolled?

Did the study avoid inappropriate exclusions?

Yes

Low

Low

\section{DOMAIN 2: Index Test All tests}

Were the index test results interpreted without knowledge Yes

of the results of the reference standard?

If a threshold was used, was it pre-specified?

Yes

Point-of-care ultrasonography for diagnosing thoracoabdominal injuries in patients with blunt trauma (Review) 
Emery 2001 (Continued)

Was the qualification of the US operator appropriate? Yes

Was the US hardware (i.e. generation, manufacturer, Yes probe, etc.) up to date?

Was the US protocol (i.e. 'classic' FAST) appropriate? Yes

Are there concerns that the definition or performance of No the index test (i.e. POC US of trauma) do not match gen-

erally accepted, established, or practiced rules or recommendations?

\section{DOMAIN 3: Reference Standard}

\section{Is the reference standards likely to correctly classify the Yes} target condition?

\section{Were the reference standard results interpreted without Yes}

knowledge of the results of the index tests?

Was the qualification of the doctors (i.e. radiologists, sur- Yes geons, etc.) determining the reference standard appropriate?

Was the reference imaging standard (i.e. MDCT-rows (4 to Yes $\geq 256$ slices), contrast-imaging, etc.) up to date?

Are there concerns that the definition or performance of the reference tests (e.g. CT, MRI, laparatomy, thoracoto-

\section{DOMAIN 4: Flow and Timing}

Was there an appropriate interval between index test and Yes reference standard?

\begin{tabular}{ll}
\hline Did all patients receive the same reference standard? & Yes \\
\hline Were all patients included in the analysis? & Yes \\
\hline $\begin{array}{l}\text { Did all participants receive a reference standard? (Risk of } \\
\text { partial verification bias) }\end{array}$ & Yes \\
\hline
\end{tabular}

\section{Fox 2011}

\section{Study characteristics}

Patient sampling Prospective study


Fox 2011 (Continued)

Patient characteristics and setting
Study location: California, USA

Study period: 2004 to 2007

Care setting: level I trauma centre

Mass casualty: no

Participants enrolled: 475

Participants included in analysis: 357: 230 men and 127 women

Age: range 0 to 17 years (more graduation in study)

Type of injury: blunt abdominal trauma

Injury severity: IQR ISS 4 to 12

Haemodynamic stability: not reported

Inclusion criteria: patients with blunt trauma as falls, motor vehicle crashes, automobile versus pedestrian collisions, non-accidental blunt trauma, and battery

Exclusion criteria: no consent to study inclusion, no confirmation of FAST results by $\mathrm{CT}$, penetrating injuries, accidental blunt trauma

US protocol: the FAST exams were performed by emergency medicine residents ( $62 \%$ of total, $51 \%$ of total by third-year emergency medicine residents), attending emergency physicians (21\%), and US fellows and surgeons ( $8 \%$ each)

Hardware used: B+K Hawk 2102 (Copenhagen, Denmark), SonoSite Titan, or SonoSite Micromaxx (Bothell, WA) US machine with 3.5- to 5.0$\mathrm{MHz}$ curved array (Hawk), 2- to 4-MHz convex array (Titan), or 2- to 4$\mathrm{MHz}$ phased array transducers

Description of imaging technique: locations evaluated for free fluid: around the heart and 3 areas of the abdominal-pelvic cavity (hepatorenal, splenorenal, suprapubic)

\section{Target condition: free fluid and air}

Reference standard: CECT (technique specifications not reported) or laparotomy

Description of technique: by protocol, all eligible participants had FAST at arrival followed by CT of the abdomen and pelvis within $30 \mathrm{~min}$ utes, or underwent laparotomy. All participants had CT with IV contrast; oral contrast was routinely used for the first 18 months of the study, but not thereafter. 
Fox 2011 (Continued)

Item

\section{DOMAIN 1: Patient Selection}

Was a consecutive or random sample of patients enrolled?
No

Did the study avoid inappropriate exclusions? Yes

Unclear

Low

\section{DOMAIN 2: Index Test All tests}

Were the index test results interpreted without knowledge Yes of the results of the reference standard?

\begin{tabular}{ll}
\hline If a threshold was used, was it pre-specified? & Yes \\
\hline Was the qualification of the US operator appropriate? & Yes \\
\hline $\begin{array}{l}\text { Was the US hardware (i.e. generation, manufacturer, } \\
\text { probe, etc.) up to date? }\end{array}$ & Yes \\
\hline $\begin{array}{l}\text { Was the US protocol (i.e. 'classic' FAST) appropriate? } \\
\text { Are there concerns that the definition or performance of }\end{array}$ & No \\
the index test (i.e. POC US of trauma) do not match gen- \\
erally accepted, established, or practiced rules or recom- \\
mendations?
\end{tabular}

\section{DOMAIN 3: Reference Standard}

Is the reference standards likely to correctly classify the Yes target condition?

Were the reference standard results interpreted without Yes knowledge of the results of the index tests?

Was the qualification of the doctors (i.e. radiologists, sur- Yes geons, etc.) determining the reference standard appropriate?

Was the reference imaging standard (i.e. MDCT-rows (4 to Unclear $\geq 256$ slices), contrast-imaging, etc.) up to date?

Are there concerns that the definition or performance of the reference tests (e.g. CT, MRI, laparatomy, thoracotomy, autopsy, etc.) do not match generally accepted, established, or practiced rules or recommendations?

\section{DOMAIN 4: Flow and Timing}


Fox 2011 (Continued)

Was there an appropriate interval between index test and Yes reference standard?

\begin{tabular}{ll}
\hline Did all patients receive the same reference standard? & No \\
\hline Were all patients included in the analysis? & Yes \\
\hline
\end{tabular}

Did all participants receive a reference standard? (Risk of Yes

partial verification bias)

Low

\section{Friese 2007}

\section{Study characteristics}

\begin{tabular}{ll}
\hline Patient sampling & Retrospective study \\
\hline Patient characteristics and setting & Study location: Texas, USA
\end{tabular}

Patient characteristics and setting

Study period: November 2003 to February 2005

Care setting: level I trauma centre

Mass casualty: no

Participants enrolled: 126

Participants included in analysis: 96: 47 men and 49 women

Age: mean age $42.3 \pm 22.5$ years

Type of injury: pelvic trauma

Injury severity: not reported

Haemodynamic stability: increased risk for haemorrhage

Inclusion criteria: "the trauma registry was queried for all patients admitted to the trauma service through our emergency department during the study period with the diagnosis of pelvic fracture and the presence of at least one of the following risk factors for haemorrhage: age 55 years, the presence of haemorrhagic shock (systolic blood pressure $100 \mathrm{~mm} \mathrm{Hg}$ ), or an unstable fracture pattern. Additional inclusion criteria were the use of the FAST examination to evaluate the peritoneal space for the presence of haemoperitoneum on arrival to the emergency department and a confirmation of the presence or absence of haemoperitoneum by laparotomy or abdominopelvic computed tomography (CT) scan."

Exclusion criteria: "DPL performed before confirmatory evaluation and treatment at an outside facility before arrival at our emergency department."

US protocol: all FAST examinations were performed by surgery residents (postgraduate year 3 or higher) who were required to complete the American College of Surgeons course on the use of ultrasound in the acute setting. The trauma bay protocol specifies that all FAST examinations were performed as an adjunct to the primary trauma survey, which meant that the FAST examination was completed within 5 to 10 minutes of participant arrival in the emergency department. A true-positive FAST examination was de- 
Friese 2007 (Continued)

fined as free intraperitoneal fluid detected on US and haemoperitoneum confirmed at laparotomy or abdominopelvic CT scan. A true-negative FAST examination was defined as no free fluid noted on abdominal US and the absence of haemoperitoneum noted at laparotomy or abdominopelvic CT scan. A false-positive FAST examination was defined as free intraperitoneal fluid detected on US and the absence of haemoperitoneum on laparotomy or abdominopelvic CT scan. A false-negative FAST examination was defined as no free intraperitoneal fluid detected on abdominal US and haemoperitoneum found at laparotomy or abdominopelvic CT scan.

Hardware used: portable 2102 HAWK ultrasound unit (B\&K Medical, Willmington, MA) with a low-frequency $(3.5-\mathrm{MHz})$ transducer and a curvilinear array

Description of imaging technique: standard 4-view FAST examination

Target condition and reference stan$\operatorname{dard}(\mathrm{s})$
Target condition: free fluid and air

Reference standard: CT (technical specifications not reported), laparotomy

Description of technique: all operative and abdominopelvic CT scan reports were also reviewed for documentation of the presence of haemoperitoneum. CT scan and operative findings were based on the final dictated and transcribed reports placed in the permanent medical record from the attending radiologist and attending surgeon. Special information about the technique of the reference standard was not reported.

Flow and timing

Time between US and reference standard: not reported

\section{Comparative}

\section{Notes}

\section{Methodological quality}

\begin{tabular}{llll}
\hline Item & Authors' judgement & Risk of bias & Applicability concerns \\
\hline
\end{tabular}

DOMAIN 1: Patient Selection

Was a consecutive or random sample of Unclear
patients enrolled?

Was a consecutive or random sample of Unclear

Did the study avoid inappropriate exclu- Yes sions?

Unclear High

\section{DOMAIN 2: Index Test All tests}

Were the index test results interpreted

Unclear without knowledge of the results of the reference standard?

If a threshold was used, was it pre-speci- Yes fied?

Was the qualification of the US operator Yes appropriate?

Was the US hardware (i.e. generation, manufacturer, probe, etc.) up to date? 
Friese 2007 (Continued)

\author{
Was the US protocol (i.e. 'classic' FAST) Yes \\ appropriate?
}

Are there concerns that the definition or

No performance of the index test (i.e. POC US of trauma) do not match generally accepted, established, or practiced rules or recommendations?

Low

Low

\title{
DOMAIN 3: Reference Standard
}

Is the reference standards likely to cor-

Yes rectly classify the target condition?

\begin{abstract}
Were the reference standard results inter- Unclear preted without knowledge of the results of the index tests?
\end{abstract}

Was the qualification of the doctors (i.e. radiologists, surgeons, etc.) determining the reference standard appropriate?

Was the reference imaging standard Unclear
(i.e. MDCT-rows ( 4 to $\geq 256$ slices), con-
trast-imaging, etc.) up to date?
Are there concerns that the definition or performance of the reference tests (e.g. $\mathrm{CT}, \mathrm{MRI}$, laparatomy, thoracotomy, autop- sy, etc.) do not match generally accepted, established, or practiced rules or recom- mendations?

\begin{tabular}{lll} 
& & Unclear \\
\hline DOMAIN 4: Flow and Timing & \\
\hline $\begin{array}{l}\text { Was there an appropriate interval be- } \\
\text { tween index test and reference standard? }\end{array}$ & Yes \\
\hline $\begin{array}{l}\text { Did all patients receive the same refer- } \\
\text { ence standard? }\end{array}$ & No \\
\hline $\begin{array}{l}\text { Were all patients included in the analysis? } \\
\text { Did all participants receive a reference }\end{array}$ & Yes \\
standard? (Risk of partial verification & \\
bias) & \\
\hline
\end{tabular}


Ghafouri 2016

\section{Study characteristics}

\begin{tabular}{ll}
\hline Patient sampling & Prospective study \\
\hline Patient characteristics and setting & Study location: Iran \\
& Study period: February 2011 to January 2012 \\
& Care setting: university hospital \\
& Mass casualty: no \\
& Participants enrolled: $102:$ gender ratio male:female $=3: 1$ \\
& Participants included in analysis: 102 \\
& Age: mean age $33 \pm 16.6$ years \\
& Type of injury: blunt abdominal trauma \\
& Injury severity: not reported \\
& Haemodynamic stability: stable and unstable conditions \\
& Inclusion criteria: all patients with abdominal blunt trauma eval- \\
uated for abdominal fluid
\end{tabular}

Exclusion criteria: not reported

US protocol: all US evaluations were performed using the FAST technique during the primary survey of ATLS guidelines

Hardware used: SonoSite180, handheld system, curvilinear probe (2 to $4 \mathrm{MHz}$ )

Description of imaging technique: positive findings included the presence of abdominal fluid in any of the abdomino-pelvic spaces

\footnotetext{
Target condition and reference standard(s)
}

Target condition: free fluid and air

Reference standard: CECT (technique specification not reported), laparotomy

Description of technique: abdominal CT with IV and oral contrast as the gold standard for all participants. Since some participants had been transferred directly to the operating room and underwent laparotomies without undergoing abdominal CT scans, a combination of laparotomy (if performed) and abdominal CT scan were used as gold standards.

\section{Comparative}

\section{Notes}

\section{Methodological quality}

\begin{tabular}{llll}
\hline Item & $\begin{array}{l}\text { Authors' judge- } \\
\text { ment }\end{array}$ & Risk of bias & $\begin{array}{l}\text { Applicability con- } \\
\text { cerns }\end{array}$ \\
\hline
\end{tabular}


Ghafouri 2016 (Continued)

\section{DOMAIN 1: Patient Selection}

\begin{tabular}{lll}
\hline Was a consecutive or random sample of patients enrolled? & Yes \\
\hline Did the study avoid inappropriate exclusions? & Yes & Low \\
\hline
\end{tabular}

\section{DOMAIN 2: Index Test All tests}

Were the index test results interpreted without knowledge of Yes the results of the reference standard?

\begin{tabular}{lc} 
If a threshold was used, was it pre-specified? & Yes \\
\hline Was the qualification of the US operator appropriate? & Yes \\
\hline $\begin{array}{l}\text { Was the US hardware (i.e. generation, manufacturer, probe, } \\
\text { etc.) up to date? }\end{array}$ & Yes
\end{tabular}

Was the US protocol (i.e. 'classic' FAST) appropriate? Yes

Are there concerns that the definition or performance of the index test (i.e. POC US of trauma) do not match generally accepted, established, or practiced rules or recommendations?

\begin{tabular}{lll}
\hline Low & Low \\
\hline
\end{tabular}

\section{DOMAIN 3: Reference Standard}

Is the reference standards likely to correctly classify the target Yes condition?

Were the reference standard results interpreted without knowl- Yes edge of the results of the index tests?

Was the qualification of the doctors (i.e. radiologists, surgeons, Unclear etc.) determining the reference standard appropriate?

Was the reference imaging standard (i.e. MDCT-rows ( 4 to $\geq 256 \quad$ Unclear slices), contrast-imaging, etc.) up to date?

Are there concerns that the definition or performance of the ref- No erence tests (e.g. CT, MRI, laparatomy, thoracotomy, autopsy, etc.) do not match generally accepted, established, or practiced rules or recommendations?

\begin{tabular}{lll}
\hline & Unclear & Low \\
\hline DOMAIN 4: Flow and Timing & & \\
\hline $\begin{array}{l}\text { Was there an appropriate interval between index test and refer- } \\
\text { ence standard? }\end{array}$ & Yes \\
\hline Did all patients receive the same reference standard? & Yes \\
\hline Were all patients included in the analysis?
\end{tabular}


Ghafouri 2016 (Continued)

Did all participants receive a reference standard? (Risk of par- Unclear tial verification bias)

\section{Unclear}

Hsu 2007

\section{Study characteristics}

\begin{tabular}{ll}
\hline Patient sampling & Prospective study \\
\hline Patient characteristics and setting & Study location: Australia \\
& Study period: September 1999 to December 2004 \\
& Care setting: emergency department of teaching hospital \\
& Mass casualty: no \\
& Participants enrolled: 463 \\
& Participants included in analysis: $357: 71 \%$ men and $29 \%$ women \\
Age: mean age $37 \pm 23$ years & $\begin{array}{l}\text { Type of injury: blunt truncal trauma } \\
\text { Injury severity: } \text { not reported }\end{array}$ \\
& Haemodynamic stability: stable and unstable conditions \\
Inclusion criteria: any trauma patient who came through the \\
emergency department underwent a FAST study if the appropriate \\
personnel were available
\end{tabular}

Exclusion criteria: those who did not have US results confirmed by either CT or laparotomy

US protocol: all participants were examined with US in areas mentioned below. No attempt was made to delineate solid organ injury.

Hardware used: B-K Medical Panther (Scan Medics, Chatswood NSW Australia, distributor for B-K Medical, Herlev Denmark) US system with a 3.5- to 5.0-MHz curvilinear transducer

Description of imaging technique: the standard 4 areas were examined for the presence of free intraperitoneal fluid, namely: Morison's pouch, the splenorenal recess, the pelvis, and the pericardial area

Target condition and reference standard(s)

Target condition: free fluid and air

Reference standard: CT (technical specification not reported), laparotomy

Description of technique: all included studies with the radiologist's formal CT scan report, or the surgeon's operative notes. There 
Hsu 2007 (Continued)

was no quantitative measurement of free fluid seen on CT scans at the study institution.

Flow and timing

Time between US and reference standard: not reported

Comparative

\section{Notes}

\section{Methodological quality}

\begin{tabular}{llll}
\hline Item Authors' judgement & Risk of bias & $\begin{array}{l}\text { Applicability con- } \\
\text { cerns }\end{array}$
\end{tabular}

\section{DOMAIN 1: Patient Selection}

\begin{tabular}{lcc}
\hline Was a consecutive or random sample of patients enrolled? & No \\
\hline Did the study avoid inappropriate exclusions? & Yes & Unclear Low
\end{tabular}

\section{DOMAIN 2: Index Test All tests}

Were the index test results interpreted without knowledge of Unclear the results of the reference standard?

\begin{tabular}{lll}
\hline If a threshold was used, was it pre-specified? & Yes \\
\hline Was the qualification of the US operator appropriate? & No & \\
\hline $\begin{array}{l}\text { Was the US hardware (i.e. generation, manufacturer, probe, } \\
\text { etc.) up to date? }\end{array}$ & Yes & Yes \\
\hline $\begin{array}{l}\text { Was the US protocol (i.e. 'classic' FAST) appropriate? } \\
\begin{array}{l}\text { Are there concerns that the definition or performance of the } \\
\text { index test (i.e. POC US of trauma) do not match generally ac- } \\
\text { cepted, established, or practiced rules or recommendations? }\end{array}\end{array}$ \\
\hline
\end{tabular}

\section{DOMAIN 3: Reference Standard}

Is the reference standards likely to correctly classify the target Yes condition?

Were the reference standard results interpreted without Unclear
knowledge of the results of the index tests?

Was the qualification of the doctors (i.e. radiologists, sur- Yes geons, etc.) determining the reference standard appropriate?

Was the reference imaging standard (i.e. MDCT-rows (4 to $\geq \quad$ Unclear 256 slices), contrast-imaging, etc.) up to date?

Are there concerns that the definition or performance of the Yes reference tests (e.g. CT, MRI, laparatomy, thoracotomy, au- 
Hsu 2007 (Continued)

topsy, etc.) do not match generally accepted, established, or practiced rules or recommendations?

Unclear

High

\section{DOMAIN 4: Flow and Timing}

Was there an appropriate interval between index test and ref- Yes erence standard?

\begin{tabular}{ll}
\hline Did all patients receive the same reference standard? & Yes \\
\hline Were all patients included in the analysis? & Yes \\
\hline $\begin{array}{l}\text { Did all participants receive a reference standard? (Risk of par- } \\
\text { tial verification bias) }\end{array}$ & Yes \\
\hline
\end{tabular}

\section{Iqbal 2014}

\section{Study characteristics}

\begin{tabular}{ll}
\hline Patient sampling Cross-sectional validation study \\
\hline
\end{tabular}

Patient characteristics and setting

Study location: Islamabad, Pakistan

Study period: January 2010 to December 2011

Care setting: emergency department of a teaching hospital

Mass casualty: no

Participants enrolled: $100: 88 \%$ men and $12 \%$ women

Participants included in analysis: 100

Age: mean age of $31.52 \pm 16.79$ years (range 2 to 71 years)

Type of injury: blunt abdominal trauma

Injury severity: not reported

Haemodynamic stability: stable and unstable conditions

Inclusion criteria: patients with a history of or mechanism of injury suggestive of blunt abdominal injury, any subjective complaints of abdominal or flank pain, presence of abdominal tenderness to palpation, presence of abdominal distension, external signs of injury such as abdominal wall bruising ('seat-belt sign'), or elicitation of any peritoneal signs

Exclusion criteria: patients with penetrating abdominal injuries

US protocol: FAST was performed as part of the primary or secondary survey of the participant in the emergency department. Using a portable US machine, the scans were performed and interpreted by a radiologist within 1 hour of the par- 
Iqbal 2014 (Continued)

ticipant arriving at the hospital. All participants in the study underwent a FAST scan.

Hardware used: live 2-D mode (rapid B-mode) and transducer frequencies between 3 and $6 \mathrm{MHz}$ (no technique specification reported)

Description of imaging technique: bedside US is an integral component of trauma management that is primarily used to detect free intraperitoneal fluid after blunt trauma. The trauma US examination focuses on dependent intraperitoneal sites where blood is most likely to accumulate: the hepatorenal space (i.e. Morison's pouch), the splenorenal recess, and the inferior portion of the intraperitoneal cavity (including pouch of Douglas).

Target condition and reference standard(s)
Target condition: free fluid and air

Reference standard: CT (technique specification not reported), laparotomy (DPL)

Description of technique: all participants underwent either CT or ELAP depending on their clinical condition. FAST examination results, which were recorded as positive or negative and were compared with the findings on CT or exploratory laparotomy, which were considered definitive. CT was recommended for the evaluation of haemodynamically stable participants. Haemodynamically unstable participants were evaluated further for other causes of haemorrhage by DPL and, if indications were fulfilled, underwent a laparotomy.

Time between US and reference standard: not reported

Flow and timing

Time between US and reference standard: not reported

\section{Comparative}

Notes

\section{Methodological quality}

\begin{tabular}{llll}
\hline Item & Authors' judgement & Risk of bias & Applicability concerns \\
\hline DOMAIN 1: Patient Selection & & \\
\hline $\begin{array}{l}\text { Was a consecutive or random sample of patients } \\
\text { enrolled? }\end{array}$ & Unclear & \\
\hline Did the study avoid inappropriate exclusions? & Yes & Unclear & Low \\
\hline
\end{tabular}

\section{DOMAIN 2: Index Test All tests} Were the index test results interpreted without
knowledge of the results of the reference standard?

\begin{tabular}{ll}
\hline If a threshold was used, was it pre-specified? & Yes \\
\hline $\begin{array}{l}\text { Was the qualification of the US operator appro- } \\
\text { priate? }\end{array}$ & Yes \\
\hline
\end{tabular}
Was the US hardware (i.e. generation, manufac- Yes turer, probe, etc.) up to date?


Iqbal 2014 (Continued)

Was the US protocol (i.e. 'classic' FAST) appro- Yes priate?

Are there concerns that the definition or performance of the index test (i.e. POC US of trauma)

No do not match generally accepted, established, or practiced rules or recommendations?

\section{DOMAIN 3: Reference Standard}

Is the reference standards likely to correctly Yes classify the target condition?

\section{Were the reference standard results interprettests?

Was the qualification of the doctors (i.e. radiologists, surgeons, etc.) determining the reference standard appropriate?

\section{Was the reference imaging standard (i.e. MD-} CT-rows ( 4 to $\geq 256$ slices), contrast-imaging, etc.) up to date?

Are there concerns that the definition or perYes formance of the reference tests (e.g. CT, MRI, laparatomy, thoracotomy, autopsy, etc.) do not match generally accepted, established, or practiced rules or recommendations?

\section{DOMAIN 4: Flow and Timing}

Was there an appropriate interval between index Yes test and reference standard?

Did all patients receive the same reference stan- No dard?

\begin{tabular}{ll}
\hline Were all patients included in the analysis? & Yes \\
\hline $\begin{array}{l}\text { Did all participants receive a reference stan- } \\
\text { dard? (Risk of partial verification bias) }\end{array}$ & Yes \\
\hline
\end{tabular}

\section{Study characteristics}

\section{Patient sampling}

Prospective cohort study 


\section{Kendall 2009 (Continued)}

Patient characteristics and setting
Study location: USA

Study period: July 1998 to June 1999

Care setting: level I trauma centre

Mass casualty: no

Participants enrolled: 164

Participants included in analysis: 152: 95 men and 57 women

Age: mean age 34 (range 6 to 91 years)

Type of injury: blunt abdominal trauma

Injury severity: not reported

Haemodynamic stability: not reported

Inclusion criteria: a convenience sample of patients who presented to the emergency department following blunt abdominal trauma and who subsequently received CT scans of the chest or abdomen during their evaluations

Exclusion criteria: transferred from another facility with known solid organ injury; if the CT was interrupted or not completed; if performing the secondary US would delay necessary patient care; or if the trauma was not a blunt mechanism

\section{Index test: FAST}

US protocol: US examinations were performed by emergency medicine residents or attending physicians experienced in the use of US for detecting haemoperitoneum. Ultrasonographers determined the presence or absence of liver or spleen injury prospectively. The specific purpose of the secondary US was to evaluate the liver and splenic parenchyma for solid organ injury. The secondary US consisted of longand short-axis scans through both organs.

Hardware used: Toshiba SSH-140A (Toshiba, San Francisco, CA) with a 3.75- $\mathrm{MHz}$ phased array transducer

Description of imaging technique: a 4-view US examination to detect haemoperitoneum or pericardial effusion

Reference standard: CT (technique specification not reported)

Description of technique: the criterion diagnostic standard was made using CT. All CT interpretations were performed by attending radiologists who were blinded to the results of the secondary US examination.

Comparative

\section{Methodological quality}


Kendall 2009 (Continued)

Item

DOMAIN 1: Patient Selection

Was a consecutive or random sample of patients enrolled?
No

Yes
Unclear

Low

\section{DOMAIN 2: Index Test All tests}

Were the index test results interpreted without knowledge Unclear of the results of the reference standard?

\begin{tabular}{|c|c|c|}
\hline If a threshold was used, was it pre-specified? & Yes & \\
\hline Was the qualification of the US operator appropriate? & No & \\
\hline $\begin{array}{l}\text { Was the US hardware (i.e. generation, manufacturer, } \\
\text { probe, etc.) up to date? }\end{array}$ & Yes & \\
\hline Was the US protocol (i.e. 'classic' FAST) appropriate? & Yes & \\
\hline \multirow{2}{*}{$\begin{array}{l}\text { Are there concerns that the definition or performance of } \\
\text { the index test (i.e. POC US of trauma) do not match gen- } \\
\text { erally accepted, established, or practiced rules or recom- } \\
\text { mendations? }\end{array}$} & No & \\
\hline & Unclear & Low \\
\hline
\end{tabular}

\section{DOMAIN 3: Reference Standard}

Is the reference standards likely to correctly classify the Yes target condition?

Were the reference standard results interpreted without Yes knowledge of the results of the index tests?

Was the qualification of the doctors (i.e. radiologists, sur- Yes geons, etc.) determining the reference standard appropriate?

Was the reference imaging standard (i.e. MDCT-rows (4 to Unclear $\geq 256$ slices), contrast-imaging, etc.) up to date?

Are there concerns that the definition or performance of the reference tests (e.g. CT, MRI, laparatomy, thoracotomy, autopsy, etc.) do not match generally accepted, established, or practiced rules or recommendations?

\section{DOMAIN 4: Flow and Timing}




\section{Kendall 2009 (Continued)}

Was there an appropriate interval between index test and Yes reference standard?

\begin{tabular}{lcl}
\hline Did all patients receive the same reference standard? & Yes \\
\hline Were all patients included in the analysis? & Yes \\
\hline $\begin{array}{l}\text { Did all participants receive a reference standard? (Risk of } \\
\text { partial verification bias) }\end{array}$ & Yes \\
\hline & & Low \\
\hline
\end{tabular}

\section{Kumar 2015}

\section{Study characteristics}

Patient sampling

Patient characteristics and setting
Prospective study

Study location: New Delhi, India

Study period: April 2004 to May 2006

Care setting: hospital emergency department

Mass casualty: no

Participants enrolled: 50: 42 men and 8 women

Participants included in analysis: 50

Age: mean age 28.62 years (range 3 to 65 years)

Type of injury: blunt abdominal trauma

Injury severity: not reported

Haemodynamic stability: stable and unstable conditions

Inclusion criteria: 50 consecutive patients with history of blunt abdominal trauma presenting in the emergency department

Exclusion criteria: those who presented with unrecordable blood pressure or in shock, with an indication for an immediate laparotomy

\section{Index test: FAST}

US protocol: a FAST examination was done during initial resuscitation in the emergency department. Haemodynamically stable participants with a positive FAST for free fluid underwent an abdominal CT scan with IV and oral contrast. Participants who were haemodynamically unstable and not responding to resuscitation, or if there was any other indication for laparotomy, underwent immediate surgery without any further investigation. FAST was performed by the resident radiologist in the emergency department before CECT, laparotomy, or autopsy.

Hardware used: Sonoline Versa Pro, Siemens, Germany, curvilinear/sector probe of $3.5 \mathrm{MHz}$ 
Kumar 2015 (Continued)

Description of imaging technique: FAST is a limited US examination that is aimed primarily at the identification of the presence of free intraperitoneal or pericardial fluid.

Target condition and reference standard(s)

Target condition: free fluid and air

Reference standard: CECT (technique specification not reported), laparotomy, autopsy

Description of technique: for participants in whom non-operative management was decided, a CECT scan of the abdomen and pelvis including lower chest was performed to confirm the findings of FAST. No further details of the CECT technique were reported. The CECT scan was done in haemodynamically stable participants or those who responded to resuscitation and for whom a non-operative management was planned with no immediate indication for laparotomy.

Flow and timing

Time between US and reference standard: not reported

Comparative

Notes

\section{Methodological quality}

\begin{tabular}{llll}
\hline Item Authors' judgement & Risk of bias & $\begin{array}{l}\text { Applicability con- } \\
\text { cerns }\end{array}$
\end{tabular}

DOMAIN 1: Patient Selection

Was a consecutive or random sample of patients en- Yes rolled?

\begin{tabular}{llll}
\hline Did the study avoid inappropriate exclusions? & Yes & Low Lw & Low \\
\hline
\end{tabular}

\section{DOMAIN 2: Index Test All tests}

Were the index test results interpreted without knowl- Unclear edge of the results of the reference standard?

\begin{tabular}{ll}
\hline If a threshold was used, was it pre-specified? & Yes \\
\hline Was the qualification of the US operator appropriate? & Yes \\
\hline
\end{tabular}

Was the US hardware (i.e. generation, manufacturer, Yes probe, etc.) up to date?

Was the US protocol (i.e. 'classic' FAST) appropriate? Yes

Are there concerns that the definition or performance of the index test (i.e. POC US of trauma) do not match generally accepted, established, or practiced rules or recommendations?

\begin{tabular}{lll}
\hline Low & Low \\
\hline
\end{tabular}


Kumar 2015 (Continued)

\section{DOMAIN 3: Reference Standard}

Is the reference standards likely to correctly classify Yes the target condition?

\section{Were the reference standard results interpreted with- Unclear} out knowledge of the results of the index tests?

\section{Was the qualification of the doctors (i.e. radiologists, Unclear} surgeons, etc.) determining the reference standard appropriate?

Was the reference imaging standard (i.e. MDCT-rows ( 4 to $\geq 256$ slices), contrast-imaging, etc.) up to date?

Unclear

Are there concerns that the definition or performance of the reference tests (e.g. CT, MRI, laparatomy, thoracotomy, autopsy, etc.) do not match generally accepted, established, or practiced rules or recommendations?

\section{DOMAIN 4: Flow and Timing}

Was there an appropriate interval between index test Yes and reference standard?

Did all patients receive the same reference standard? No

\begin{tabular}{ll}
\hline Were all patients included in the analysis? & Yes \\
\hline
\end{tabular}

Did all participants receive a reference standard? Yes

(Risk of partial verification bias)

Kärk 2012

\section{Study characteristics}

\section{Patient sampling}

Patient characteristics and setting

\author{
Retrospective study
}

Study location: Denmark

Study period: January 2003 to December 2010

Care setting: hospital emergency department

Mass casualty: no

Participants enrolled: 405: gender specification not reported

\section{Participants included in analysis: 118}

Age: not reported for 118 participants 
Kärk 2012 (Continued)

Type of injury: blunt abdominal trauma, liver injury

Injury severity: not reported

Haemodynamic stability: stable and unstable conditions

Inclusion criteria: this study included all patients admitted to the institution registered with the ICD-10 code S36.1 'Injury of the liver or gallbladder' as defined by the WHO, especially abdominal blunt trauma patients

Exclusion criteria: patients with iatrogenic lesions of the biliary system

US protocol: the majority of participants were examined by radiologists trained in general abdominal US, and a minority by surgeons trained in FAST

Hardware used: not reported

Description of imaging technique: recorded findings of intraperitoneal free fluid (if possible in 4 different areas: Morison's pouch in the right upper quadrant, the perisplenic space in the left upper quadrant, the pericardium of the epigastric region, and the pelvis)

Target condition and reference standard(s)

Flow and timing
Target condition: free fluid and air

Reference standard: CT (technique specification not reported), explorative laparotomy

Description of technique: not reported

Comparative

$$
\text { Notes }
$$

\section{Methodological quality}

\begin{tabular}{llll}
\hline Item & $\begin{array}{l}\text { Authors' judge- } \\
\text { ment }\end{array}$ & Risk of bias & $\begin{array}{l}\text { Applicability con- } \\
\text { cerns }\end{array}$ \\
\hline
\end{tabular}

\section{DOMAIN 1: Patient Selection}

\begin{tabular}{llll}
\hline Was a consecutive or random sample of patients enrolled? & Unclear & \\
\hline Did the study avoid inappropriate exclusions? & Yes & Unclear & High \\
\hline
\end{tabular}

\section{DOMAIN 2: Index Test All tests}

Were the index test results interpreted without knowledge of Unclear the results of the reference standard? 
Kärk 2012 (Continued)

Was the qualification of the US operator appropriate?

Was the US hardware (i.e. generation, manufacturer, probe, Unclear etc.) up to date?

Was the US protocol (i.e. 'classic' FAST) appropriate?

Are there concerns that the definition or performance of the index test (i.e. POC US of trauma) do not match generally accept-

ed, established, or practiced rules or recommendations?

No

\begin{tabular}{lll}
\hline & Unclear & Low \\
\hline DOMAIN 3: Reference Standard &
\end{tabular}

Is the reference standards likely to correctly classify the target Yes condition?

Were the reference standard results interpreted without knowl- Unclear edge of the results of the index tests?

Was the qualification of the doctors (i.e. radiologists, surgeons, Unclear etc.) determining the reference standard appropriate?

Was the reference imaging standard (i.e. MDCT-rows (4 to $\geq 256 \quad$ Unclear slices), contrast-imaging, etc.) up to date?

Are there concerns that the definition or performance of the ref- Yes erence tests (e.g. CT, MRI, laparatomy, thoracotomy, autopsy, etc.) do not match generally accepted, established, or practiced rules or recommendations?

\begin{tabular}{lll}
\hline DOMAIN 4: Flow and Timing & Unclear & High \\
\hline $\begin{array}{l}\text { Was there an appropriate interval between index test and refer- } \\
\text { ence standard? }\end{array}$ & Yes \\
\hline Did all patients receive the same reference standard? & No \\
\hline Were all patients included in the analysis? & Yes \\
\hline $\begin{array}{l}\text { Did all participants receive a reference standard? (Risk of par- } \\
\text { tial verification bias) }\end{array}$ & Yes \\
\hline
\end{tabular}

Low

\section{McElveen 1997}

\section{Study characteristics}

\begin{tabular}{ll}
\hline Patient sampling & Prospective study \\
\hline Patient characteristics and setting & Study location: Virginia, USA
\end{tabular}


Study period: not reported

Care setting: level I trauma centre

Mass casualty: no

Participants enrolled: 82

Participants included in analysis: 70

Age: mean age 38 (range 1 to 82 years)

Type of injury: blunt abdominal trauma

Injury severity: not reported

Haemodynamic stability: not reported

Inclusion criteria: 82 consecutive patients with a diagnosis of blunt abdominal trauma presenting to the trauma resuscitation room when either of the 2 authors was present

Exclusion criteria: not reported

Index test: FAST

US protocol: US was performed concurrently with inital resusciation and prior to other studies

Hardware used: $3.0-\mathrm{MHz}$ sector probe, Johnson \& Johnson Ultrasound $280 \mathrm{SL}$

Description of imaging technique: the examination consisted of an evaluation of the pericardial area, right upper quadrant, left upper quadrant, and pouch of Douglas. In addition, the liver and spleen were evaluated for parenchymal injury. The examination was considered to be positive if there was any free intraperitoneal fluid present or visceral injury was identified. The examination was considered to be negative if no intraperitoneal fluid or visceral injury was seen.

Target condition and reference standard(s)
Target condition: free fluid and air, organ injury

Reference standard: CT (technique specification not reported), laparotomy (DPL)

Description of technique: criteria for positive CT findings were visceral injury or fluid in the peritoneal cavity. More technical specifications of $\mathrm{CT}$ were not reported.

Time between US and reference standard: not reported

Comparative

\section{Notes}

\section{Methodological quality}


McElveen 1997 (Continued)

Was a consecutive or random sample of patients enrolled? Yes

Did the study avoid inappropriate exclusions? Yes

Low

Low

\section{DOMAIN 2: Index Test All tests}

Were the index test results interpreted without knowledge of Unclear the results of the reference standard?

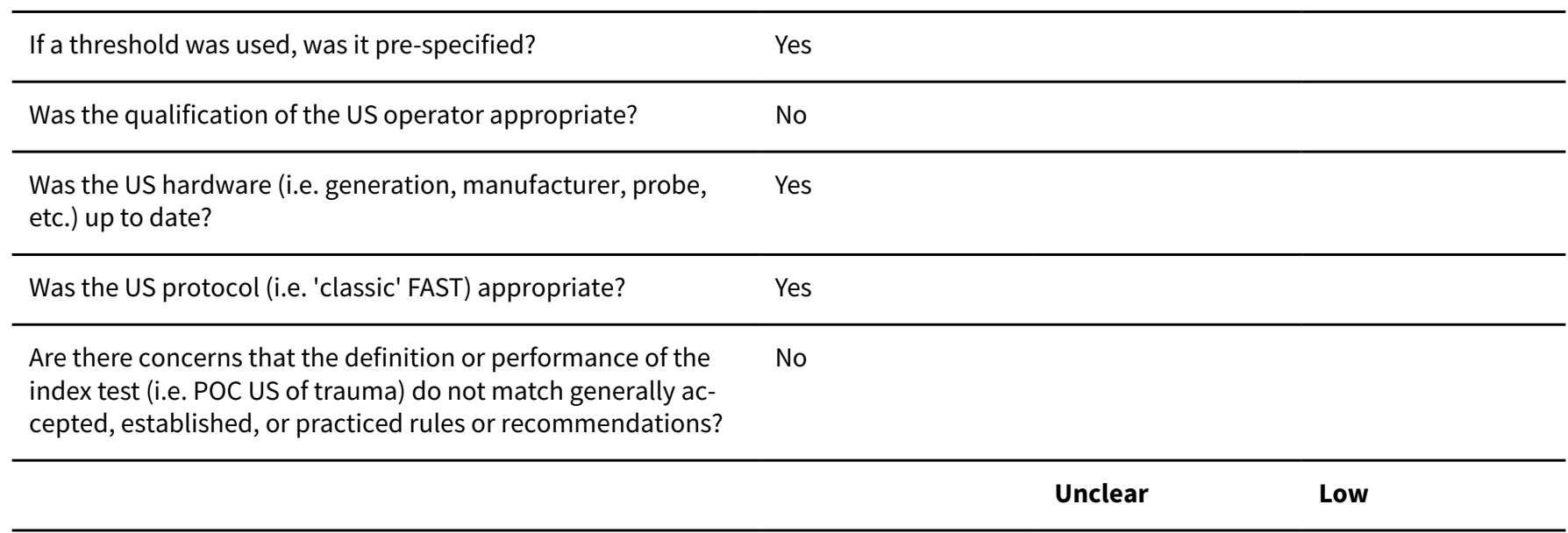

\section{DOMAIN 3: Reference Standard}

Is the reference standards likely to correctly classify the target Yes condition?

Were the reference standard results interpreted without Unclear knowledge of the results of the index tests?

Was the qualification of the doctors (i.e. radiologists, surgeons, etc.) determining the reference standard appropriate?

Was the reference imaging standard (i.e. MDCT-rows (4 to $\geq \quad$ Unclear 256 slices), contrast-imaging, etc.) up to date?

Are there concerns that the definition or performance of the Yes reference tests (e.g. CT, MRI, laparatomy, thoracotomy, autopsy, etc.) do not match generally accepted, established, or practiced rules or recommendations?

\begin{tabular}{lll}
\hline High & Unclear & High \\
\hline
\end{tabular}

\section{DOMAIN 4: Flow and Timing}

Was there an appropriate interval between index test and ref- Yes erence standard?

Did all patients receive the same reference standard? No

Were all patients included in the analysis?

Yes 
McElveen 1997 (Continued)

Did all participants receive a reference standard? (Risk of par- No tial verification bias)

High

McKenney 1994

\section{Study characteristics}

\section{Patient sampling}

Patient characteristics and setting
Prospective study

Study location: USA

Study period: October 1992 to June 1993

Care setting: level 1 trauma centre

Mass casualty: no

Participants enrolled: 200: 142 men and 58 women

Participants included in analysis: 145

Age: mean age 37 (range 11 to 92 years)

Type of injury: blunt abdominal trauma

Injury severity: $\mathrm{GCS} \leq 12$

Haemodynamic stability: stable conditions

Inclusion criteria: patients with blunt abdominal trauma and trauma criteria (systolic blood pressure $<90 \mathrm{mmHg}$ ) - see Table 1 in study

Exclusion criteria: none

Index tests Index test: FAST

US protocol: emergency US performed in the resuscitation room at the discretion of the attending surgeon. The US study was performed by a radiology fellow, an attending physician, or a technologist.

Hardware used: Accuson $128 \mathrm{X}$ P/10 (Mountain View, CA), Toshiba $140 \mathrm{~A}$ (Norcross, GA), or an ATL Mark (Seattle, WA) with a 3.5-MHz sector or curvilinear transducer

Description of imaging technique: the US examination consisted of evaluation of the subphrenic space, subhepatic space (Morison's pouch), paracolic gutters, and the pelvis for evidence of free intraperitoneal fluid. The liver and spleen were evaluated for parenchymal injury.

Target condition and reference standard(s)
Target condition: free fluid and air, organ injury

Reference standard: CT (technique specification not reported), DPL, laparotomy 
Description of technique: criteria for positive CT findings were visceral injury or significant fluid in the peritoneal cavity. All studies were interpreted by a radiologist either from real-time images or from hard copies.

Flow and timing

Time between US and reference standard: not reported

Comparative

$$
\text { Notes }
$$

\section{Methodological quality}

$\begin{array}{lll}\text { Item Authors'judgement } & \text { Risk of bias } & \begin{array}{l}\text { Applicability con- } \\ \text { cerns }\end{array}\end{array}$

\section{DOMAIN 1: Patient Selection}

\begin{tabular}{ll}
\hline Was a consecutive or random sample of patients enrolled? & Unclear \\
\hline Did the study avoid inappropriate exclusions? & Yes
\end{tabular}

\begin{tabular}{l}
\hline Unclear \\
Low
\end{tabular}

\section{DOMAIN 2: Index Test All tests}

Were the index test results interpreted without knowledge of Unclear the results of the reference standard?

\begin{tabular}{lll}
\hline If a threshold was used, was it pre-specified? & Yes \\
\hline Was the qualification of the US operator appropriate? & No & \\
\hline $\begin{array}{l}\text { Was the US hardware (i.e. generation, manufacturer, probe, } \\
\text { etc.) up to date? }\end{array}$ & Yes & Yes \\
\hline Was the US protocol (i.e. 'classic' FAST) appropriate? & No \\
\hline $\begin{array}{l}\text { Are there concerns that the definition or performance of the } \\
\text { index test (i.e. POC US of trauma) do not match generally ac- } \\
\text { cepted, established, or practiced rules or recommendations? }\end{array}$ & \\
\hline
\end{tabular}

\section{DOMAIN 3: Reference Standard}

Is the reference standards likely to correctly classify the target Yes condition?

Were the reference standard results interpreted without Unclear
knowledge of the results of the index tests?

knowledge of the results of the index tests?

Was the qualification of the doctors (i.e. radiologists, surYes geons, etc.) determining the reference standard appropriate?

Was the reference imaging standard (i.e. MDCT-rows (4 to $\geq \quad$ Unclear 256 slices), contrast-imaging, etc.) up to date? 
McKenney 1994 (Continued)

Are there concerns that the definition or performance of the

Yes reference tests (e.g. CT, MRI, laparatomy, thoracotomy, autopsy, etc.) do not match generally accepted, established, or practiced rules or recommendations?

Unclear

High

\section{DOMAIN 4: Flow and Timing}

Was there an appropriate interval between index test and ref- Yes erence standard?

\begin{tabular}{ll}
\hline Did all patients receive the same reference standard? & No \\
\hline Were all patients included in the analysis? & Yes \\
\hline $\begin{array}{l}\text { Did all participants receive a reference standard? (Risk of par- } \\
\text { tial verification bias) }\end{array}$ & Yes \\
\hline
\end{tabular}

\section{Menichini 2015}

\section{Study characteristics}

\begin{tabular}{ll}
\hline Patient sampling & Retrospective study \\
\hline
\end{tabular}

Patient characteristics and setting

Study location: Rome, Italy

Study period: October 2012 to October 2013

Care setting: department of emergency radiology

Mass casualty: no

Participants enrolled: $73: 51$ boys and 22 girls

Participants included in analysis: 73

Age: mean age $8.7 \pm 2.8$ years (range not reported)

Type of injury: low-energy blunt abdominal trauma (minor trauma)

Injury severity: not reported

Haemodynamic stability: stable conditions

Inclusion criteria: male or female, aged 0 to 16 years, haemodynamically stable children with a history of minor blunt abdominal trauma

Exclusion criteria: > 16 years of age, haemodynamical instability, history of major trauma

US protocol: 73 participants with a history of minor trauma, haemodynamic stability, and at least 1 positive finding at baseline US such as abdominal free fluid, perirenal fluid collection, signs of hepatic, splenic, or renal injury were subjected to both CEUS and CEMDCT. CEUS was performed immediately after baseline US. 
Hardware used: Acuson Sequoia 512 Ultrasound System (Siemens, Germany), equipped with both curved- and linear-array probes

Description of imaging technique: the study was conducted to detect the presence of free intraperitoneal fluid in the perihepatic area, Morison pouch, epigastric region, perisplenic region, paracolic gutters, and Douglas pouch, and the presence of perirenal fluid collection. Intra-abdominal organs were evaluated specifically for evidence of injury. Adequate US technology consisting of contrast-specific software that operates in real time at a low mechanical index (pulse inversion technology) was applied. $2 \times 1.2 \mathrm{~mL}$ boluses of second-generation blood pool contrast agent (SonoVue, Bracco, Italy) were administered through a 20-gauge catheter in the antecubital vein, followed by saline $(0.9 \%)$. An abdominal scan of 3 minutes followed each bolus, starting with the right and left kidney, liver and pancreas, and finally the spleen. A traumatic lesion was identified as the presence of a hypoechoic area that persisted unchanged during all the acquisition phases, with a subcapsular distribution in the case of haematoma, or a parenchymal localisation in the case of lacerations. The presence of intralesional hyperechoic spots was interpreted as a sign of active bleeding.

Target condition and reference stan$\operatorname{dard}(\mathrm{s})$
Target condition: free fluid and air, organ injury

Reference standard: CE-MDCT, MDCT 16 scanner (LightSpeed 16, GE Healthcare, USA)

Description of technique: the scanning parameters were as follows: 100 to $250 \mathrm{mAs}$ (applied with the care-dose technique and with a medium value of $115 \mathrm{mAs}$ ), 100 to 120 $\mathrm{kV}$ (according to physical build), 2.5-mm collimation, $13.5 \mathrm{~mm} / \mathrm{s}$ table, and 1- $\mathrm{mm}$ reconstruction interval. A dose of $2.5 \mathrm{~mL} / \mathrm{kg}$ of non-ionic contrast agent (Xenetix 350, Guerbet, France) was injected at a rate of $1.5 \mathrm{~mL} / \mathrm{s}$ to $2 \mathrm{~mL} / \mathrm{s}$. Arterial phase is performed with an acquisition delay of $40 \mathrm{~s}$; a venous phase is routinely performed with 70-second delay; late phase ( 5 minutes) was performed only in case of suspected urinary tract lesion. The presence of a parenchymal bleeding was defined as the presence of hyperechoic/hyperdense postcontrast intralesional spots.

\section{Comparative}

Notes

Methodological quality

\begin{tabular}{llll}
\hline Item & Authors' judgement & Risk of bias & Applicability concerns \\
\hline
\end{tabular}

\section{DOMAIN 1: Patient Selection}

Was a consecutive or random sample of Yes patients enrolled?

Did the study avoid inappropriate exclu-

Yes sions?

\begin{tabular}{llll}
\hline High & Low \\
\hline
\end{tabular}

\section{DOMAIN 2: Index Test All tests}

Were the index test results interpreted without knowledge of the results of the reference standard?
Unclear 
Menichini 2015 (Continued)

If a threshold was used, was it pre-speci- Yes fied?

Was the qualification of the US operator Yes appropriate?

Was the US hardware (i.e. generation, Yes manufacturer, probe, etc.) up to date?

Was the US protocol (i.e. 'classic' FAST) Yes appropriate?

Are there concerns that the definition or performance of the index test (i.e. POC

US of trauma) do not match generally accepted, established, or practiced rules or recommendations?

\section{DOMAIN 3: Reference Standard}

Is the reference standards likely to cor- Yes rectly classify the target condition?

Were the reference standard results inter- Yes preted without knowledge of the results of the index tests?

Was the qualification of the doctors (i.e. Yes radiologists, surgeons, etc.) determining the reference standard appropriate?
Was the reference imaging standard (i.e. MDCT-rows ( 4 to $\geq 256$ slices), con- trast-imaging, etc.) up to date?
Are there concerns that the definition or performance of the reference tests (e.g. $\mathrm{CT}, \mathrm{MRI}$, laparatomy, thoracotomy, autop- sy, etc.) do not match generally accepted, established, or practiced rules or recom- mendations?

\begin{tabular}{lll}
\hline DOMAIN 4: Flow and Timing & Low & High \\
\hline $\begin{array}{l}\text { Was there an appropriate interval be- } \\
\text { tween index test and reference standard? }\end{array}$ & Yes \\
\hline $\begin{array}{l}\text { Did all patients receive the same refer- } \\
\text { ence standard? }\end{array}$ & No \\
\hline Were all patients included in the analysis? & Yes \\
\hline
\end{tabular}


Menichini 2015 (Continued)

Did all participants receive a reference standard? (Risk of partial verification bias)

\section{High}

\section{Nandipati 2011}

\section{Study characteristics}

Patient sampling Prospective study

Patient characteristics and setting

Study location: Queens, USA

Study period: June 2007 to May 2008

Care setting: level I trauma centre

Mass casualty: no

Participants enrolled: 204: 152 men and 52 women

Participants included in analysis: 159

Age: mean age $43.01 \pm 19.5$ years (range not reported)

Type of injury: polytrauma, blunt and penetrating trauma

Injury severity: ISS $12.5 \pm 5.3$

Haemodynamic stability: not reported

Inclusion criteria: patients with polytrauma, blunt and penetrating trauma to the chest or thoraco-abdominal area

Exclusion criteria: patients who had chest tube placement without sonogram or CXR, or penetrating abdominal and extremity injuries

US protocol: EFAST was performed in all participants having a FAST examination as a part of their secondary survey. Chest radiography and CT scan were performed after the initial primary and secondary survey. Senior resident (level V) or attending on the trauma team familiar with the principles of the FAST examination who had attended a formal US course performed the EFAST examination.

Hardware used: $7.5-\mathrm{MHz}$ linear probe, no further technique specifications reported

Description of imaging technique: participants were kept in a supine position, and the anterior thorax was examined with the probe placed in the second intercostal space in the midclavicular line. Bilateral ultrasonographic images were obtained and compared. Pneumothorax was considered when the absence of both lung-sliding and comet-tail artefacts was noted.

Target condition and reference standard(s)

Target condition: free fluid and air

Reference standard: CT (technique specification not reported), CXR 
Nandipati 2011 (Continued)

Description of technique: not reported

Flow and timing

Time between US and reference standard: not reported

Comparative

Notes

\section{Methodological quality}

$\begin{array}{llll}\text { Item } & \text { Authors' judgement } & \text { Risk of bias } & \begin{array}{l}\text { Applicability con- } \\ \text { cerns }\end{array}\end{array}$

\section{DOMAIN 1: Patient Selection}

\begin{tabular}{ll}
\hline Was a consecutive or random sample of patients enrolled? & Unclear \\
\hline Did the study avoid inappropriate exclusions? & Yes \\
\hline
\end{tabular}

\begin{tabular}{l}
\hline Unclear \\
Low
\end{tabular}

\section{DOMAIN 2: Index Test All tests}

Were the index test results interpreted without knowledge of Unclear the results of the reference standard?

\begin{tabular}{ll}
\hline If a threshold was used, was it pre-specified? & Yes \\
\hline Was the qualification of the US operator appropriate? & Yes
\end{tabular}

Was the US hardware (i.e. generation, manufacturer, probe, Yes etc.) up to date?

Was the US protocol (i.e. 'classic' FAST) appropriate?

Are there concerns that the definition or performance of the index test (i.e. POC US of trauma) do not match generally ac-

\section{DOMAIN 3: Reference Standard}

Is the reference standards likely to correctly classify the tar- Yes get condition?

Were the reference standard results interpreted without Unclear knowledge of the results of the index tests?

Was the qualification of the doctors (i.e. radiologists, sur- Unclear geons, etc.) determining the reference standard appropriate?

Was the reference imaging standard (i.e. MDCT-rows ( 4 to $\geq \quad$ Unclear 256 slices), contrast-imaging, etc.) up to date?

Are there concerns that the definition or performance of the No reference tests (e.g. CT, MRI, laparatomy, thoracotomy, au- 
Nandipati 2011 (Continued)

topsy, etc.) do not match generally accepted, established, or practiced rules or recommendations?

\begin{tabular}{lll}
\hline & & Unclear \\
\hline DOMAIN 4: Flow and Timing & \\
\hline $\begin{array}{l}\text { Was there an appropriate interval between index test and } \\
\text { reference standard? }\end{array}$ & Yes \\
\hline $\begin{array}{l}\text { Did all patients receive the same reference standard? } \\
\text { Were all patients included in the analysis? }\end{array}$ & Yes \\
\hline $\begin{array}{l}\text { Did all participants receive a reference standard? (Risk of } \\
\text { partial verification bias) }\end{array}$ & \\
\hline
\end{tabular}

\section{Ojaghi 2014}

\section{Study characteristics}

Patient sampling Prospective study

Patient characteristics and setting

\section{Study location: Iran}

Study period: winter of 2013

Care setting: emergency department of a hospital

Mass casualty: no

Participants enrolled: 163

Participants included in analysis: 150: 124 men and 26 women

Age: not reported

Type of injury: multiple trauma, chest trauma

Injury severity: ESI1 and ESI2

Haemodynamic stability: not reported

Inclusion criteria: patients with severe multiple trauma based on the mechanism of injury, or their history and examination findings of suspected chest injuries, and chest CT scan according to an ATLS algorithm. The mechanisms of injury included: car rollover, being thrown out of the vehicle, frontal impact, compression of the chest with the steering wheel or dashboard, severe side impact, fall, or acceleration/deceleration injury.

Exclusion criteria: those who underwent a tube thoracostomy before US due to their unstable clinical situation or for any other reason, such as a lack of access to US at the time of admission 
Ojaghi 2014 (Continued)

US protocol: participants were evaluated according to the ATLS algorithm, and examination findings were recorded following initial evaluations, an emergency medicine specialist performed chest US to detect pneumothorax and haemothorax.

Hardware used: General Electric E200 US with 2 types of probes, namely: a curve probe of 5-MHz frequency for haemothorax assessment and a linear probe of 6.5- to 9-MHz frequency for pneumothorax assessment

Description of imaging technique: US images of the lung are built with air artefacts as the air stops the beam, however this artefact varies when it is in the pleural space. In a normal lung view, pleural movement along the parietal and visceral sides is called lung sliding, which can easily be seen with US. This characteristic is also known as the gliding sign. Moreover, sharp resonance appears during ventilation at the border of the pleura and lung, known as a comet-tail artefact. Trapped air in the pleural space prevents visualisation of lung sliding signs and comet-tail artefacts, therefore, based on these findings, pneumothorax can be detected with US. US permits the detection of amounts of loculated pleural fluid as small as $20 \mathrm{~mL}$, which cannot be identified by X-rays, which are only capable of detecting volumes > $50 \mathrm{~mL}$.

Reference standard: CT (technique specification not reported), CXR

Description of technique: assigned radiologists reported both CT scan and portable CXR results, while a specialist performed US. These 2 groups of examiners were unaware of each other's results. No further specifications of technique were reported.

Flow and timing

Time between US and reference standard: not reported

\section{Comparative}

Notes

\section{Methodological quality}

\begin{tabular}{llll}
\hline Item Authors' judgement $\quad$ Risk of bias $\quad$ Applicability concerns & Aut
\end{tabular}

\section{DOMAIN 1: Patient Selection}

Was a consecutive or random sample of pa- No tients enrolled?

Did the study avoid inappropriate exclusions? No

\begin{tabular}{lll}
\hline High & Low \\
\hline
\end{tabular}

DOMAIN 2: Index Test All tests

Were the index test results interpreted without knowledge of the results of the reference standard?

\begin{tabular}{ll}
\hline If a threshold was used, was it pre-specified? & Yes \\
\hline $\begin{array}{l}\text { Was the qualification of the US operator ap- } \\
\text { propriate? }\end{array}$ & Yes \\
\hline
\end{tabular}


Ojaghi 2014 (Continued)

Was the US hardware (i.e. generation, manu- Yes facturer, probe, etc.) up to date?

Was the US protocol (i.e. 'classic' FAST) ap- $\quad$ Yes propriate?

Are there concerns that the definition or performance of the index test (i.e. POC US of trauma) do not match generally accepted, established, or practiced rules or recommendations?

\begin{tabular}{lll}
\hline Low & Low \\
\hline
\end{tabular}

\section{DOMAIN 3: Reference Standard}

Is the reference standards likely to correctly Yes classify the target condition?

Were the reference standard results interpret- Yes ed without knowledge of the results of the index tests?

\section{Was the qualification of the doctors (i.e. radi- Yes} ologists, surgeons, etc.) determining the reference standard appropriate?

Was the reference imaging standard (i.e. MD- Unclear CT-rows ( 4 to $\geq 256$ slices), contrast-imaging, etc.) up to date?

Are there concerns that the definition or performance of the reference tests (e.g. CT, MRI, laparatomy, thoracotomy, autopsy, etc.) do not match generally accepted, established, or practiced rules or recommendations?

\begin{tabular}{lll}
\hline & Low & Low \\
\hline DOMAIN 4: Flow and Timing &
\end{tabular}

Was there an appropriate interval between in- Yes dex test and reference standard?

Did all patients receive the same reference Yes standard?

\begin{tabular}{ll}
\hline Were all patients included in the analysis? & Yes \\
\hline $\begin{array}{l}\text { Did all participants receive a reference stan- } \\
\text { dard? (Risk of partial verification bias) }\end{array}$ & Yes \\
\hline
\end{tabular}


Smith 2010

\section{Study characteristics}

\section{Patient sampling}

Patient characteristics and setting
Prospective study

Study location: Africa

Study period: January to December 2008

Care setting: hospital emergency department

Mass casualty: no

Participants enrolled: 91 (gender not reported)

Participants included in analysis: 52

Age: not reported

Type of injury: thoracal or abdominal trauma

Injury severity: not reported

Haemodynamic stability: stable and unstable conditions

Inclusion criteria: all patients presenting to the emergency department who had sustained abdominal or thoracic trauma

Exclusion criteria: not reported

\section{Index test: FAST}

US protocol: FAST scan findings were subsequently supported by either CT scanning or laparotomy. Where this was not indicated, findings were verified by a second qualified emergency department ultrasonographer repeating the FAST scan. All cases were documented with indication for scan, result, and final method of confirmation with any discrepancies in findings. Scans were recorded as positive or negative for free intra-abdominal or pericardial fluid.

Hardware used: Aloka SSD 500 B-scan US machine (Aloka, Japan) with a 3.5-MHz abdominal probe

Description of imaging technique: scans were performed on the supine participant. Right upper quadrant, left upper quadrant, pericardial and pelvic views were obtained according to FAST scanning principles.

\footnotetext{
Target condition and reference standard(s)
}

Target condition: free fluid and air

Reference standard: CT (technique specification not reported), laparotomy, second US examination

Description of technique: scans were confirmed by CT in 31 cases $(43.1 \%)$ and laparotomy in 17 cases (23.6\%). The remaining 24 cases $(33.3 \%)$ were rescanned by a second qualified ultrasonographer and observed clinically.
Time between US and reference standard: not reported

\section{Notes}


Smith 2010 (Continued)

Methodological quality

\begin{tabular}{llll}
\hline Item Authors' judgement & Risk of bias & $\begin{array}{l}\text { Applicability con- } \\
\text { cerns }\end{array}$ \\
\hline
\end{tabular}

\section{DOMAIN 1: Patient Selection}

Was a consecutive or random sample of patients enrolled? No

Did the study avoid inappropriate exclusions? Yes

\begin{tabular}{lll}
\hline & Unclear & Low \\
\hline DOMAIN 2: Index Test All tests & & \\
\hline
\end{tabular}

Were the index test results interpreted without knowledge of Unclear the results of the reference standard?

\begin{tabular}{|c|c|}
\hline If a threshold was used, was it pre-specified? & Yes \\
\hline Was the qualification of the US operator appropriate? & Yes \\
\hline $\begin{array}{l}\text { Was the US hardware (i.e. generation, manufacturer, probe, } \\
\text { etc.) up to date? }\end{array}$ & Yes \\
\hline Was the US protocol (i.e. 'classic' FAST) appropriate? & Yes \\
\hline \multirow[t]{2}{*}{$\begin{array}{l}\text { Are there concerns that the definition or performance of the } \\
\text { index test (i.e. POC US of trauma) do not match generally ac- } \\
\text { cepted, established, or practiced rules or recommendations? }\end{array}$} & No \\
\hline & Low \\
\hline
\end{tabular}

\section{DOMAIN 3: Reference Standard}

Is the reference standards likely to correctly classify the tar- Yes get condition?

Were the reference standard results interpreted without Unclear

knowledge of the results of the index tests?

Was the qualification of the doctors (i.e. radiologists, sur- Unclear geons, etc.) determining the reference standard appropriate?

Was the reference imaging standard (i.e. MDCT-rows (4 to $\geq \quad$ Unclear 256 slices), contrast-imaging, etc.) up to date?

Are there concerns that the definition or performance of the Yes reference tests (e.g. CT, MRI, laparatomy, thoracotomy, autopsy, etc.) do not match generally accepted, established, or practiced rules or recommendations?

Unclear

High

\section{DOMAIN 4: Flow and Timing}


Smith 2010 (Continued)

Was there an appropriate interval between index test and Yes reference standard?

Did all patients receive the same reference standard?

No

Were all patients included in the analysis? Yes

Did all participants receive a reference standard? (Risk of

partial verification bias)

Yes

Low

\section{Soudack 2004}

\section{Study characteristics}

\section{Patient sampling}

Patient characteristics and setting

\section{Retrospective study}

Study location: Israel

Study period: May 1998 to January 2000

Care setting: hospital emergency department

Mass casualty: no

Participants enrolled: 313: 204 boys and 109 girls

Participants included in analysis: 109

Age: mean age 7.1 years (range 0 to 17 years)

Type of injury: blunt abdominal trauma

Injury severity: not reported

Haemodynamic stability: stable and unstable conditions

Inclusion criteria: all children included in the analysis were listed consecutively in the trialists' database. Data pertaining to the mechanism of injury, clinical findings, diagnostic imaging, and management were analysed.

Exclusion criteria: not reported

\section{Index test: FAST}

US protocol: during daytime working hours, FAST examination was performed by a staff radiologist trained in sonography; at other times, it was performed by a radiology resident who had received at least 6 months' theoretical and clinical training in sonography in emergency settings

Hardware used: Tosbee scanner (Toshiba, Tokyo, Japan) equipped with a convex phased-array transducer with a frequency of $3.5 \mathrm{MHz}$ or $7 \mathrm{MHz}$, depending on size of participant

Description of imaging technique: sonographic examination consisted of evaluation of 4 anatomic areas for the presence or absence of free fluid: the midline infrasternal or second left intercostal space (to detect haemopericardium), Morison's pouch or the hepatorenal space, the splenorenal space, and the pelvis. Visualisation of the retrovesical space required distension of the bladder before the FAST exami- 
Soudack 2004 (Continued)

nation. When the bladder was empty, retrograde filling with saline was done using a Foley catheter. A positive FAST result was defined as a study with evidence of free peritoneal fluid plus a report of parenchymal injury or retroperitoneal fluid.

Target condition and reference standard(s)

Target condition: free fluid and air

Reference standard: CECT, using a Helical Twin Flash scanner (Elscint, Haifa, Israel) until 1999 and a multislice Mx8000 scanner (Marconi, Cleveland, OH) thereafter, ELAP

Description of technique: CT examination performed when, despite a negative FAST result, clinical signs of abdominal injury, such as abrasions of the torso skin, distension of the abdomen, signs of peritoneal injury, or haematuria were present. CT examination of the abdomen performed if requested by the trauma surgeon. Participants who had CT examination of the chest were more likely to undergo abdominal CT examination, depending on the discretion of the trauma surgeon. A contrast medium was administered orally or through a nasogastric tube. An IV non-ionic iodinised contrast medium $(2 \mathrm{~mL} / \mathrm{kg}$ ) was administered. The CT scanning protocol consisted of a 5.5-mm slice thickness at a 5-mm interval, a voltage of $120 \mathrm{kV}$, and a current of $200 \mathrm{~mA}$. The CT examinations were interpreted by the radiology resident and the senior radiologist on call.

Flow and timing

Time between US and reference standard: not reported

\section{Comparative}

Notes

\section{Methodological quality}

\begin{tabular}{llll}
\hline Item & Authors' judgement & Risk of bias & Applicability concerns \\
\hline
\end{tabular}

\section{DOMAIN 1: Patient Selection}

Was a consecutive or random sample of pa- Yes tients enrolled?

Did the study avoid inappropriate exclusions? Yes

Low Low

\section{DOMAIN 2: Index Test All tests}

Were the index test results interpreted with- Unclear
out knowledge of the results of the reference standard?

\begin{tabular}{ll}
\hline If a threshold was used, was it pre-specified? & Yes \\
\hline $\begin{array}{l}\text { Was the qualification of the US operator ap- } \\
\text { propriate? }\end{array}$ & Yes \\
\hline $\begin{array}{l}\text { Was the US hardware (i.e. generation, manu- } \\
\text { facturer, probe, etc.) up to date? }\end{array}$ \\
$\begin{array}{l}\text { Was the US protocol (i.e. 'classic' FAST) ap- } \\
\text { propriate? }\end{array}$
\end{tabular}




\section{Soudack 2004 (Continued)}

Are there concerns that the definition or performance of the index test (i.e. POC US of trauma) do not match generally accepted, established, or practiced rules or recommendations?

\begin{tabular}{|c|c|c|c|}
\hline & & Low & Low \\
\hline \multicolumn{4}{|l|}{ DOMAIN 3: Reference Standard } \\
\hline $\begin{array}{l}\text { Is the reference standards likely to correctly } \\
\text { classify the target condition? }\end{array}$ & Yes & & \\
\hline $\begin{array}{l}\text { Were the reference standard results interpret- } \\
\text { ed without knowledge of the results of the in- } \\
\text { dex tests? }\end{array}$ & Unclear & & \\
\hline $\begin{array}{l}\text { Was the qualification of the doctors (i.e. radi- } \\
\text { ologists, surgeons, etc.) determining the ref- } \\
\text { erence standard appropriate? }\end{array}$ & Yes & & \\
\hline $\begin{array}{l}\text { Was the reference imaging standard (i.e. MD- } \\
\text { CT-rows ( } 4 \text { to } \geq 256 \text { slices), contrast-imaging, } \\
\text { etc.) up to date? }\end{array}$ & Yes & & \\
\hline \multirow[t]{2}{*}{$\begin{array}{l}\text { Are there concerns that the definition or per- } \\
\text { formance of the reference tests (e.g. CT, MRI, } \\
\text { laparatomy, thoracotomy, autopsy, etc.) do } \\
\text { not match generally accepted, established, or } \\
\text { practiced rules or recommendations? }\end{array}$} & Yes & & \\
\hline & & Low & High \\
\hline
\end{tabular}

\section{DOMAIN 4: Flow and Timing}

Was there an appropriate interval between in- Yes dex test and reference standard?

Did all patients receive the same reference No standard?

Were all patients included in the analysis? Yes

Did all participants receive a reference stan- Yes

dard? (Risk of partial verification bias)

\section{Low}

\section{Talari 2015}

\section{Study characteristics}

\begin{tabular}{ll}
\hline Patient sampling & Cross-sectional study \\
\hline
\end{tabular}

Patient characteristics and setting

Study location: Iran 
Talari 2015 (Continued)

Study period: not reported

Care setting: hospital emergency department

Mass casualty: no

Participants enrolled: 200: 133 men and 67 women

Participants included in analysis: 200

Age: mean age $29.6 \pm 18.3$ years (range not reported)

Type of injury: blunt abdominal trauma

Injury severity: GCS $12.9 \pm 3.4$

Haemodynamic stability: stable conditions

Inclusion criteria: haemodynamically stable patients with severe blunt trauma referred to the emergency department of Shahid Beheshti hospital in Kashan, Iran. Severe trauma was assumed if the mechanism of trauma was high energy (falls more than $3 \mathrm{~m}$, motor vehicle accident with a speed more than $50 \mathrm{~km} / \mathrm{h}$, crush injuries, rollover, and pedestrian accidents), or if severe injuries such as vertebral or pelvic fractures were detected.

Exclusion criteria: haemodynamically unstable patients (systolic blood pressure $<90 \mathrm{mmHg}$ ), penetrating trauma, pregnant women, and those with underlying diseases associated with intra-abdominal fluid (cirrhosis, congestive heart failure)

\section{Index test: FAST}

US protocol: evaluation by FAST in the supine position

Hardware used: Medison ultrasound (V20) curvilinear 3.5- to 5-microhertz transducer

Description of imaging technique: the Huang classification was used for measurement of the intra-abdominal fluid. 1 point was given for each intra-abdominal region (Douglas pouch, hepatorenal recess, perisplenic and paracolic gutters) if free intra-abdominal fluid was present; 2 points were given if $>2 \mathrm{~mL}$ free fluid was seen in the hepatorenal recess and Douglas pouch, or floating bowel loops were observed.

\footnotetext{
Target condition and reference standard(s)
}

Target condition: free fluid and air

Reference standard: CECT, Toshiba-Astion (no further specification of technique reported)

Description of technique: US followed by abdominopelvic $\mathrm{CT}$ for all participants from the diaphragm dome to pubis symphysis with IV contrast (Visipaque $270 \mathrm{mg}$ vial, $1 \mathrm{~mL} / \mathrm{kg}$ ). The CT scan was reported by a second radiologist who was blinded to the results of the US. 
Talari 2015 (Continued)

Item

\section{DOMAIN 1: Patient Selection}

Was a consecutive or random sample of patients en- No rolled?

Did the study avoid inappropriate exclusions? No

High Low

\section{DOMAIN 2: Index Test All tests}

Were the index test results interpreted without knowl- Yes

edge of the results of the reference standard?

\begin{tabular}{ll}
\hline If a threshold was used, was it pre-specified? & Yes \\
\hline Was the qualification of the US operator appropriate? & Yes
\end{tabular}

Was the US hardware (i.e. generation, manufacturer, Yes probe, etc.) up to date?

Was the US protocol (i.e. 'classic' FAST) appropriate? Yes

Are there concerns that the definition or performance No of the index test (i.e. POC US of trauma) do not match generally accepted, established, or practiced rules or recommendations?

\begin{tabular}{lll}
\hline Low & Low \\
\hline
\end{tabular}

\section{DOMAIN 3: Reference Standard}

Is the reference standards likely to correctly classify Yes the target condition?

Were the reference standard results interpreted with- Yes out knowledge of the results of the index tests?

Was the qualification of the doctors (i.e. radiologists, Unclear surgeons, etc.) determining the reference standard appropriate?

Was the reference imaging standard (i.e. MDCT-rows Yes ( 4 to $\geq 256$ slices), contrast-imaging, etc.) up to date?

Are there concerns that the definition or performance of the reference tests (e.g. CT, MRI, laparatomy, thoracotomy, autopsy, etc.) do not match generally accepted, established, or practiced rules or recommendations?

\begin{tabular}{lcc}
\hline & Low & Low \\
\hline DOMAIN 4: Flow and Timing &
\end{tabular}

\section{DOMAIN 4: Flow and Timing}


Talari 2015 (Continued)

Was there an appropriate interval between index test Yes and reference standard?

\begin{tabular}{ll}
\hline Did all patients receive the same reference standard? & Yes \\
\hline Were all patients included in the analysis? & Yes \\
\hline
\end{tabular}

Did all participants receive a reference standard?

Yes

(Risk of partial verification bias)

Low

Todd Miller 2003

\section{Study characteristics}

Patient sampling

Patient characteristics and setting
Prospective study

Study location: USA

Study period: October 2001 to June 2002

Care setting: level I trauma centre

Mass casualty: no

Participants enrolled: 372 : gender not reported

Participants included in analysis: 359

Age: mean age 38 years (range 2 to 93 years)

Type of injury: blunt abdominal trauma

Injury severity: not reported

Haemodynamic stability: stable conditions

Inclusion criteria: all haemodynamically stable patients with suspected blunt abdominal injury (i.e. abdominal pain or mechanism of injury consistent with the production of an intra-abdominal injury)

Exclusion criteria: inadequate FAST examination attributable to physical build

Index tests

\section{Index test: FAST}

US protocol: all participants with abdominal pain or mechanism of injury consistent with the production of an intra-abdominal injury underwent a 4-view FAST examination at the completion of the secondary survey during initial trauma evaluation. The presence of intra-abdominal injury without free abdominal fluid (true-negative FAST examination), retroperitoneal injury, bony injury (lower thoracic, lumbar, or pelvic fractures), and clinically significant "incidentalomas" were also recorded.

Hardware used: $3.5 \mathrm{MHz}$, SonoSite, Bothell, WA

Description of imaging technique: FAST examinations were recorded as positive or negative for 3 abdominal views and considered inadequate if any 1 of the 3 abdominal views could not be obtained. A true-positive FAST examination was defined as free fluid detected in any 1 of the 3 abdominal views confirmed 
Todd Miller 2003 (Continued)

by CT scan, whereas a true-negative FAST examination was defined as no free abdominal fluid by FAST examination or CT scan. A false-negative FAST examination was defined as any negative US examination with a subsequent CT scan that showed the presence of intra-abdominal fluid. A false-positive FAST examination was defined as one in which the FAST examination was felt to demonstrate free abdominal fluid, but the CT scan was negative for free fluid.

Target condition and reference standard(s)

Target condition: free fluid and air, organ injury

Reference standard: CT (technique specification not reported)

Description of technique: CT scan of the abdomen and pelvis was obtained and interpreted by an in-house radiologist within 1 hour of the FAST examination being completed. Participants underwent CT scanning of the abdomen and pelvis as a confirmatory test for the presence of intra-abdominal fluid and intra-abdominal injury.

Flow and timing

Time between US and reference standard: not reported

Comparative

Notes

\section{Methodological quality}

\begin{tabular}{llll}
\hline Item Authors' judgement & Risk of bias & Applicability concerns
\end{tabular}

\section{DOMAIN 1: Patient Selection}

Was a consecutive or random sample of patients Unclear enrolled?

\begin{tabular}{llll}
\hline Did the study avoid inappropriate exclusions? & Yes & \\
\hline & Unclear & Low \\
\hline
\end{tabular}

\section{DOMAIN 2: Index Test All tests}

Were the index test results interpreted without Yes knowledge of the results of the reference standard?

\begin{tabular}{ll}
\hline If a threshold was used, was it pre-specified? Yes \\
\hline
\end{tabular}

Was the qualification of the US operator appropri- Yes ate?

Was the US hardware (i.e. generation, manufac- Yes turer, probe, etc.) up to date?

Was the US protocol (i.e. 'classic' FAST) appropri- $\quad$ Yes ate?

Are there concerns that the definition or perforNo mance of the index test (i.e. POC US of trauma) do not match generally accepted, established, or practiced rules or recommendations? 
Todd Miller 2003 (Continued)

Low

Low

\section{DOMAIN 3: Reference Standard}

Is the reference standards likely to correctly clas- Yes

sify the target condition?

Were the reference standard results interpret- Unclear

ed without knowledge of the results of the index

tests?

Was the qualification of the doctors (i.e. radiolo-

gists, surgeons, etc.) determining the reference

standard appropriate?

Was the reference imaging standard (i.e. MD-

CT-rows ( 4 to $\geq 256$ slices), contrast-imaging, etc.) up to date?

Are there concerns that the definition or performance of the reference tests (e.g. CT, MRI, laparatomy, thoracotomy, autopsy, etc.) do not match generally accepted, established, or practiced rules or recommendations?

\section{Unclear}

\section{DOMAIN 4: Flow and Timing}

Was there an appropriate interval between index Yes test and reference standard?

Did all patients receive the same reference stan- Yes dard?

Were all patients included in the analysis? Yes

Did all participants receive a reference standard? Yes

(Risk of partial verification bias)

\section{Low}

\section{Tso 1992}

\section{Study characteristics}

\begin{tabular}{ll} 
Patient sampling $\quad$ Prospective study \\
\hline
\end{tabular}

Patient characteristics and setting

\section{Study location: USA}

Study period: 8 consecutive months in the 1990 academic year

Care setting: level I trauma centre

Mass casualty: no

Participants enrolled: 163: gender ratio male:female 3:1 


\section{Participants included in analysis: 163}

Age: mean age 34 years (range 2 to 93 years)

Type of injury: blunt abdominal trauma

Injury severity: ISS 13, GCS 14

Haemodynamic stability: stable conditions

Inclusion criteria: patients admitted to trauma centre through the admitting area and judged to require DPL or CT for evaluation of intra-abdominal injury

Exclusion criteria: hypotension, suspected severe head injury, indications for immediate laparotomy, and the discretion of the attending traumatologist

US protocol: participants evaluated by real-time sector scanning sonography, within 1 hour of admission, by surgical trauma fellows

Hardware used: Siemens Sonoline SL-2 using either 3- or 5-MHz transducers

Description of imaging technique: the pelvis, the paracolic gutters, and the subhepatic space were studied for evidence of free intraperitoneal fluid. Positive sonography studies showed free peritoneal or extraperitoneal fluid or organ disruption.

Reference standard: CT (technique specification not reported), DPL, laparotomy

Description of technique: positive CT scans showed free peritoneal or extraperitoneal fluid or organ disruption

Comparative

$$
\text { Notes }
$$

\section{Methodological quality}

\begin{tabular}{|c|c|c|c|}
\hline Item & $\begin{array}{l}\text { Authors' judge- } \\
\text { ment }\end{array}$ & Risk of bias & $\begin{array}{l}\text { Applicability con- } \\
\text { cerns }\end{array}$ \\
\hline
\end{tabular}

\section{DOMAIN 1: Patient Selection}

Was a consecutive or random sample of patients enrolled?

Did the study avoid inappropriate exclusions?
Yes

Yes

\begin{tabular}{lll}
\hline Low & Low \\
\hline
\end{tabular}

\section{DOMAIN 2: Index Test All tests}


Tso 1992 (Continued)

Were the index test results interpreted without knowledge of Yes the results of the reference standard?

\begin{tabular}{lll}
\hline If a threshold was used, was it pre-specified? & Yes & No \\
\hline Was the qualification of the US operator appropriate? & Yes & \\
\hline $\begin{array}{l}\text { Was the US hardware (i.e. generation, manufacturer, probe, } \\
\text { etc.) up to date? }\end{array}$ & Yes \\
\hline $\begin{array}{l}\text { Was the US protocol (i.e. 'classic' FAST) appropriate? } \\
\begin{array}{l}\text { Are there concerns that the definition or performance of the in- No } \\
\text { dex test (i.e. POC US of trauma) do not match generally accept- } \\
\text { ed, established, or practiced rules or recommendations? }\end{array}\end{array}$ \\
\hline
\end{tabular}

\section{DOMAIN 3: Reference Standard}

Is the reference standards likely to correctly classify the target Yes condition?

Were the reference standard results interpreted without knowl- Unclear edge of the results of the index tests?

Was the qualification of the doctors (i.e. radiologists, surgeons, Unclear etc.) determining the reference standard appropriate?

Was the reference imaging standard (i.e. MDCT-rows ( 4 to $\geq 256$ Unclear slices), contrast-imaging, etc.) up to date?

Are there concerns that the definition or performance of the ref- Yes erence tests (e.g. CT, MRI, laparatomy, thoracotomy, autopsy, etc.) do not match generally accepted, established, or practiced rules or recommendations?

\begin{tabular}{lll}
\hline High & Unclear & High \\
\hline
\end{tabular}

\section{DOMAIN 4: Flow and Timing}

Was there an appropriate interval between index test and refer- Yes ence standard?

\begin{tabular}{lc} 
Did all patients receive the same reference standard? & No \\
\hline Were all patients included in the analysis? & Yes \\
\hline $\begin{array}{l}\text { Did all participants receive a reference standard? (Risk of par- } \\
\text { tial verification bias) }\end{array}$ & Yes
\end{tabular}

tial verification bias) 
Valentino 2010

\section{Study characteristics}

\section{Patient sampling}

Patient characteristics and setting
Prospective study

Study location: Bologna, Italy

Study period: 2004 to 2008

Care setting: hospital emergency department

Mass casualty: no

Participants enrolled: 1584

Participants included in analysis: 133: 99 men and 34 women

Age: mean age 40.2 years (range not reported)

Type of injury: blunt abdominal trauma

Injury severity: not reported

Haemodynamic stability: stable conditions

Inclusion criteria: the causes of blunt abdominal trauma were road accident, sports injury, accidental fall, or accident at work. Patients had suspected abdominal injuries with pain at palpation, bruises of the abdomen, probable fracture of the lower ribs, or presence of abdominal free fluid at FAST.

Exclusion criteria: not reported

Index tests

Index test: US, CEUS

US protocol: US and CEUS performed consecutively by 1 radiologist, after which CECT was performed by another radiologist. The latter was informed of the US/CEUS diagnosis but was blinded to the images.

Hardware used: ATL $5000 \mathrm{HDI}$ and Philips iU22 using 2e5- and 1e5-MHz convex probes

Description of imaging technique: in addition to FAST assessment, a complete study of the solid organs of the abdomen was performed in all participants to search for possible alterations. US outcome was considered positive when peritoneal free fluid or alterations in the parenchymal echo pattern consistent with traumatic injury were found. CEUS was performed after baseline US. A standard protocol was followed, and contrast agent was injected in 2 separate doses of $2.4 \mathrm{~mL}$ to permit an adequate study of the solid organs of the right upper and left quadrant. CEUS outcome was considered positive when a perfusion defect of the studied organ was found, characterised by hypoechogenicity with or without interruption of the organ profile. Where there was non-perfusion of part of or the whole organ, the finding was interpreted as a sign of vascular injury, and the passage of microbubbles outside the damaged organ was interpreted as active bleeding.

Target condition: free fluid and air, organ injury

Reference standard: CECT

Description of technique: after CEUS, CECT was performed in the venous phase before and after administration of non-ionic contrast agent. In the presence of free fluid collection, late-phase evaluation was carried out at 3 to 15 minutes to identify active bleeding or urine collection. 
Valentino 2010 (Continued)

Flow and timing

Time between US and reference standard: $<1 \mathrm{~h}$

\section{Comparative}

Notes

\section{Methodological quality}

\begin{tabular}{llll}
\hline Item & Authors' judgement & Risk of bias & Applicability concerns \\
\hline DOMAIN 1: Patient Selection & & \\
\hline $\begin{array}{l}\text { Was a consecutive or random sample of patients } \\
\text { enrolled? }\end{array}$ & No & \\
\hline Did the study avoid inappropriate exclusions? & Yes & Unclear & Low
\end{tabular}

\section{DOMAIN 2: Index Test All tests}

\section{Were the index test results interpreted without Unclear} knowledge of the results of the reference standard?

\begin{tabular}{ll}
\hline If a threshold was used, was it pre-specified? & Yes \\
\hline $\begin{array}{l}\text { Was the qualification of the US operator appropri- } \\
\text { ate? }\end{array}$ & Yes
\end{tabular}

Was the US hardware (i.e. generation, manufac- Yes turer, probe, etc.) up to date?

Was the US protocol (i.e. 'classic' FAST) appropri- Unclear ate?

\section{Are there concerns that the definition or perfor- Unclear} mance of the index test (i.e. POC US of trauma)

do not match generally accepted, established, or practiced rules or recommendations?

\section{DOMAIN 3: Reference Standard}

Is the reference standards likely to correctly clas- Yes sify the target condition?

Were the reference standard results interpreted without knowledge of the results of the index tests? 
Valentino 2010 (Continued)

Was the reference imaging standard (i.e. MD-

CT-rows ( 4 to $\geq 256$ slices), contrast-imaging, etc.)

up to date?

Are there concerns that the definition or per-

formance of the reference tests (e.g. CT, MRI, la-

paratomy, thoracotomy, autopsy, etc.) do not

match generally accepted, established, or prac-

ticed rules or recommendations?

Unclear

Low

\section{DOMAIN 4: Flow and Timing}

Was there an appropriate interval between index Yes

test and reference standard?

Did all patients receive the same reference stan- Yes

dard?

\begin{tabular}{lc}
\hline Were all patients included in the analysis? & Yes \\
\hline $\begin{array}{l}\text { Did all participants receive a reference standard? } \\
\text { (Risk of partial verification bias) }\end{array}$ & Yes \\
\hline
\end{tabular}

Low

Verbeek 2014

\section{Study characteristics}

Patient sampling Retrospective study

Patient characteristics and setting

Study location: Amsterdam, the Netherlands

Study period: January 2004 to December 2009

Care setting: level I trauma centre

Mass casualty: no

Participants enrolled: 131: 90 men and 41 women

Participants included in analysis: 120

Age: mean age 37 years (range not reported)

Type of injury: high-energy pelvic fracture

Injury severity: ISS $26 \pm 14$

Haemodynamic stability: stable and unstable conditions

Inclusion criteria: all adults with a high-energy major pelvic fracture admitted to the trauma resuscitation room. A major pelvic fracture was defined as a disruption of the pelvic ring in at least 2 places. 
Verbeek 2014 (Continued)

Exclusion criteria: isolated pelvic fracture patients, transfer patients, and patients declared dead on arrival

\section{Index test: FAST}

US protocol: initial participant assessment followed the institutional protocol and ATLS principles. According to the local imaging protocol, all highenergy trauma participants underwent chest and pelvic radiography as well as FAST within 5 minutes of arrival. FAST was performed by the trauma team radiologist (a senior radiology resident, or junior resident under supervision of an attending radiologist).

Hardware used: Aloka ProSound SSD 3500Plus (Biomedic, Almere, the Netherlands)

Description of imaging technique: a FAST result was considered positive if haemoperitoneum was detected in any of the 3 abdominal regions (left and right upper quadrant and pelvis)
Target condition: free fluid and air

Reference standard: CT (multislice CT scanner Somatom Sensation 4, Siemens Medical Systems, Erlangen, Germany)

Description of technique: a CT scan was considered positive if any haemoperitoneum (more than a physiological amount in the pelvis) was detected. The amount of haemoperitoneum was quantified as small (1 region), moderate ( 2 regions), or large (3 regions). For the purposes of this study, 2 senior radiology residents who were blinded to the FAST result reviewed all CT scans for the presence of haemoperitoneum.

Flow and timing

Time between US and reference standard: not reported

\section{Comparative}

Notes

\section{Methodological quality}

\begin{tabular}{llll}
\hline Item Authors' judgement & Risk of bias & \multicolumn{1}{c}{$\begin{array}{l}\text { Applicability con- } \\
\text { cerns }\end{array}$}
\end{tabular}

\section{DOMAIN 1: Patient Selection}

Was a consecutive or random sample of patients en- Yes
rolled?

Did the study avoid inappropriate exclusions? Yes

\begin{tabular}{|c|c|c|}
\hline & Low & High \\
\hline \multicolumn{3}{|l|}{ DOMAIN 2: Index Test All tests } \\
\hline $\begin{array}{l}\text { Were the index test results interpreted without knowl- } \\
\text { edge of the results of the reference standard? }\end{array}$ & Yes & \\
\hline If a threshold was used, was it pre-specified? & Yes & \\
\hline Was the qualification of the US operator appropriate? & Yes & \\
\hline
\end{tabular}


Verbeek 2014 (Continued)

Was the US hardware (i.e. generation, manufacturer, Yes probe, etc.) up to date?

Was the US protocol (i.e. 'classic' FAST) appropriate? Yes

Are there concerns that the definition or performance No of the index test (i.e. POC US of trauma) do not match generally accepted, established, or practiced rules or recommendations?

\begin{tabular}{lll}
\hline & Low & Low \\
\hline DOMAIN 3: Reference Standard
\end{tabular}

\section{DOMAIN 3: Reference Standard}

Is the reference standards likely to correctly classify Yes the target condition?

Were the reference standard results interpreted with- Yes out knowledge of the results of the index tests?

Was the qualification of the doctors (i.e. radiologists, Yes surgeons, etc.) determining the reference standard appropriate?

Was the reference imaging standard (i.e. MDCT-rows (4 to $\geq 256$ slices), contrast-imaging, etc.) up to date?

Are there concerns that the definition or performance of the reference tests (e.g. CT, MRI, laparatomy, thoracotomy, autopsy, etc.) do not match generally accepted, established, or practiced rules or recommendations?

\begin{tabular}{llll}
\hline & Low & Low \\
\hline DOMAIN 4: Flow and Timing & & \\
\hline $\begin{array}{l}\text { Was there an appropriate interval between index test } \\
\text { and reference standard? }\end{array}$ & Yes \\
\hline Did all patients receive the same reference standard? & No \\
\hline Were all patients included in the analysis? & Yes \\
\hline $\begin{array}{l}\text { Did all participants receive a reference standard? } \\
\text { (Risk of partial verification bias) }\end{array}$ \\
\hline
\end{tabular}

\section{Wong 2014}

\section{Study characteristics}


Wong 2014 (Continued)

Patient characteristics and setting
Study location: Singapore, Asia

Study period: January 2009 to December 2010

Care setting: emergency department of a tertiary hospital

Mass casualty: no

Participants enrolled: 476: 389 men and 87 women

Participants included in analysis: 221

Age: mean age 38.9 years (range not reported)

Type of injury: blunt abdominal trauma

Injury severity: not reported

Haemodynamic stability: not reported

Inclusion criteria: the identities of trauma patients who presented to the emergency department resuscitation room were acquired from the file and verified with the hospital's trauma registry. The performance of US was a mandated field in the trauma registry.

Exclusion criteria: penetrating trauma and burns

\section{Index test: FAST}

US protocol: FAST, no further details reported

Hardware used: not reported

Description of imaging technique: not reported

Target condition and reference standard(s)

Target condition: free fluid and air, organ injury

Reference standard: $\mathrm{CT}$, no further details reported

Description of technique: not reported

Flow and timing

Time between US and reference standard: not reported

\section{Comparative}

Notes

\section{Methodological quality}

\begin{tabular}{llll}
\hline Item & $\begin{array}{l}\text { Authors' judge- } \\
\text { ment }\end{array}$ & Risk of bias & $\begin{array}{l}\text { Applicability con- } \\
\text { cerns }\end{array}$
\end{tabular}

\section{DOMAIN 1: Patient Selection}

\begin{tabular}{lcc}
\hline Was a consecutive or random sample of patients enrolled? & No & \\
\hline Did the study avoid inappropriate exclusions? & Yes & Unclear \\
\hline
\end{tabular}


Wong 2014 (Continued)

\section{DOMAIN 2: Index Test All tests}

Were the index test results interpreted without knowledge of Unclear the results of the reference standard?

\begin{tabular}{ll} 
If a threshold was used, was it pre-specified? Yes \\
\hline
\end{tabular}

Was the qualification of the US operator appropriate?

No

Was the US hardware (i.e. generation, manufacturer, probe, Unclear etc.) up to date?

Was the US protocol (i.e. 'classic' FAST) appropriate? Unclear

Are there concerns that the definition or performance of the in- Unclear dex test (i.e. POC US of trauma) do not match generally accepted, established, or practiced rules or recommendations?

Unclear

Unclear

\section{DOMAIN 3: Reference Standard}

Is the reference standards likely to correctly classify the target Yes condition?

Were the reference standard results interpreted without knowl- Unclear edge of the results of the index tests?

Was the qualification of the doctors (i.e. radiologists, surgeons, Unclear etc.) determining the reference standard appropriate?

Was the reference imaging standard (i.e. MDCT-rows ( 4 to $\geq 256$ Unclear slices), contrast-imaging, etc.) up to date?

Are there concerns that the definition or performance of the ref- No erence tests (e.g. CT, MRI, laparatomy, thoracotomy, autopsy, etc.) do not match generally accepted, established, or practiced rules or recommendations?

\begin{tabular}{lll}
\hline DOMAIN 4: Flow and Timing & Unclear \\
\hline $\begin{array}{l}\text { Was there an appropriate interval between index test and refer- } \\
\text { ence standard? }\end{array}$ & Yes \\
\hline Did all patients receive the same reference standard? & Yes \\
\hline Were all patients included in the analysis? & Yes \\
\hline $\begin{array}{l}\text { Did all participants receive a reference standard? (Risk of par- } \\
\text { tial verification bias) }\end{array}$ & Yes \\
\hline
\end{tabular}


Zhang 2006

\section{Study characteristics}

\section{Patient sampling}

Patient characteristics and setting
Prospective study

\section{Study location: China}

Study period: September 2004 to October 2005

Care setting: hospital emergency department

Mass casualty: no

Participants enrolled: 163

Participants included in analysis: 135: 114 men and 21 women

Age: mean age $45 \pm 15$ years (range not reported)

Type of injury: multiple trauma

Injury severity: ISS $29.1 \pm 12.4$

Haemodynamic stability: stable and unstable conditions

Inclusion criteria: patients with multiple trauma in either the resuscitation room or the emergency intensive care unit were enrolled. All patients suffered from blunt trauma, including traffic accident, falls, crush injuries, and other causes.

Exclusion criteria: subcutaneous emphysema and/or cardiac arrest following probable tension pneumothorax

\section{Index test: EFAST, CXR}

US protocol: US was performed after initial rapid assessment by physical examination and essential resuscitation for participants in the resuscitation room. US was conducted in all participants admitted to the emergency intensive care unit and in hospitalised participants with impairment of lung function requiring a chest $\mathrm{CT}$ scan.

Hardware used: SSD-900, Aloka Co, Tokyo, Japan; 3.5-MHz convex probe and occasionally a 7.5-MHz linear probe

Description of imaging technique: participants were kept in a supine position and an examination of the anterior, lateral, and posterior thoraces was performed. Bilateral US images were compared, and characteristic signs (i.e. pleural line, lung sliding, comet-tail artefacts) were identified in either real-time or time-movement mode.

\footnotetext{
Target condition and reference standard(s)
}

Target condition: free fluid and air

Reference standard: CT, 16-slice spiral CT scanning unit (Volume Zoom, Siemens Co, Forchheim, Germany), CXR

Description of technique: portable chest radiography and CT scans were performed before or after US with participants in the supine position. The results of chest $\mathrm{CT}$ and radiography were interpreted by independent radiologists who were unaware of participants' conditions and the findings of US. 
Zhang 2006 (Continued)

Comparative

\section{Notes}

\section{Methodological quality}

\begin{tabular}{llll}
\hline Item & Authors' judgement & Risk of bias & $\begin{array}{l}\text { Applicability con- } \\
\text { cerns }\end{array}$ \\
\hline
\end{tabular}

\section{DOMAIN 1: Patient Selection}

Was a consecutive or random sample of patients en- Unclear rolled?

Did the study avoid inappropriate exclusions?

Yes

\begin{tabular}{lll}
\hline & Unclear & Low \\
\hline DOMAIN 2: Index Test All tests &
\end{tabular}

\section{DOMAIN 2: Index Test All tests}

Were the index test results interpreted without knowl- Yes edge of the results of the reference standard?

\begin{tabular}{lc}
\hline If a threshold was used, was it pre-specified? & Yes \\
\hline Was the qualification of the US operator appropriate? & Yes \\
\hline $\begin{array}{l}\text { Was the US hardware (i.e. generation, manufacturer, } \\
\text { probe, etc.) up to date? }\end{array}$ & Yes \\
\hline Was the US protocol (i.e. 'classic' FAST) appropriate? & Yes \\
\hline $\begin{array}{l}\text { Are there concerns that the definition or performance } \\
\text { of the index test (i.e. POC US of trauma) do not match } \\
\text { generally accepted, established, or practiced rules or } \\
\text { recommendations? }\end{array}$ & \\
\end{tabular}

\begin{tabular}{lll}
\hline & Low & Low \\
\hline DOMAIN 3: Reference Standard & &
\end{tabular}

Is the reference standards likely to correctly classify Yes the target condition?

Were the reference standard results interpreted with- Yes out knowledge of the results of the index tests?

Was the qualification of the doctors (i.e. radiologists, Yes surgeons, etc.) determining the reference standard appropriate?

Was the reference imaging standard (i.e. MDCT-rows (4 to $\geq 256$ slices), contrast-imaging, etc.) up to date? 
Zhang 2006 (Continued)

ed, established, or practiced rules or recommenda-

tions?

\begin{tabular}{lll}
\hline Low & Low \\
\hline
\end{tabular}

\section{DOMAIN 4: Flow and Timing}

Was there an appropriate interval between index test Yes and reference standard?

Did all patients receive the same reference standard? Yes

\begin{tabular}{ll}
\hline Were all patients included in the analysis? & Yes \\
\hline Did all participants receive a reference standard? & Yes
\end{tabular}

Did all participants receive a reference standard? Yes

(Risk of partial verification bias)

\section{Zhou 2012}

\section{Study characteristics}

\begin{tabular}{ll}
\hline Patient sampling $\quad$ Retrospective study \\
\hline
\end{tabular}

Patient characteristics and setting

Study location: China

Study period: 12 to 31 May 2008

Care setting: various hospitals

Mass casualty: yes (earthquake)

Participants enrolled: 2204: 1045 men and 1149 women, 19 gender unknown

Participants included in analysis: 96

Age: mean age 44.82 years (range 7 months to 103 years)

Type of injury: blunt abdominal trauma

Injury severity: not reported

Haemodynamic stability: stable and unstable conditions

Inclusion criteria: patients damaged directly and indirectly by the Wenchuan earthquake, initially examined by US within 24 hours to evaluate suspected blunt abdominal trauma at different hospitals in Sichuan province

Exclusion criteria: non-injury diseases such as stress disorder, delivery, and internal diseases

US protocol: the initial US findings were compared with the results of subsequent CT, DPL, repeated US, cystography, operation and/or autopsy, and/or the clinical course

Hardware used: not reported 
Zhou 2012 (Continued)

Description of imaging technique: US findings were considered positive if evidence of free fluid or a parenchymal injury was identified. All indeterminate parenchymal lesions were considered positive US findings. A parenchymal lesion was defined as a hyperechoic or hypoechoic area in a solid organ or a distortion of the normal echo structure of a solid organ. For the objectives of the present study, pleural and pericardial effusions were considered negative findings for abdominal injury. Positive US findings were considered true positives if initial US finding was positive, and the injury was identified by the best available reference. Positive US findings were considered false positives if injury was not confirmed at subsequent studies. In cases of medical ascites or physiologic pelvic fluid in female participants, US findings were considered as false positives because injury could not be excluded with US alone, and further investigation was required to rule out injuries. Negative US findings were considered as true negatives if findings of subsequent studies were negative or if the participant had an uneventful clinical course, or both. Negative US findings were considered as false negatives if a subsequent study revealed free fluid, haemoperitoneum, or any visceral abdominal injury.

Target condition and reference standard(s)

Target condition: free fluid and air, organ injury

Reference standard: CT (no further details reported) (DPL, repeated US, cystography, operation and/or autopsy)

Description of technique: not reported

Flow and timing

Time between US and reference standard: not reported

\section{Comparative}

Notes

\section{Methodological quality}

\begin{tabular}{llll}
\hline Item & Authors' judgement & Risk of bias & Applicability concerns \\
\hline
\end{tabular}

\section{DOMAIN 1: Patient Selection}

Was a consecutive or random sample of patients No enrolled?

Did the study avoid inappropriate exclusions? No

\begin{tabular}{lll}
\hline & High & Low \\
\hline
\end{tabular}

\section{DOMAIN 2: Index Test All tests}

Were the index test results interpreted without knowledge of the results of the reference standard?

\begin{tabular}{ll}
\hline If a threshold was used, was it pre-specified? & Yes \\
\hline $\begin{array}{l}\text { Was the qualification of the US operator appro- } \\
\text { priate? }\end{array}$ \\
\hline $\begin{array}{l}\text { Was the US hardware (i.e. generation, manufac- } \\
\text { turer, probe, etc.) up to date? }\end{array}$ \\
\hline
\end{tabular}


Zhou 2012 (Continued)

Was the US protocol (i.e. 'classic' FAST) appro- Unclear priate?

Are there concerns that the definition or perfor-

Unclear mance of the index test (i.e. POC US of trauma) do not match generally accepted, established, or practiced rules or recommendations?

\title{
DOMAIN 3: Reference Standard
}

Is the reference standards likely to correctly Unclear
classify the target condition?

Were the reference standard results interpret- Yes ed without knowledge of the results of the index tests?

Was the qualification of the doctors (i.e. radiologists, surgeons, etc.) determining the reference standard appropriate?

Unclear

Was the reference imaging standard (i.e. MDCT-rows ( 4 to $\geq 256$ slices), contrast-imaging, etc.) up to date?

Are there concerns that the definition or perYes formance of the reference tests (e.g. CT, MRI, laparatomy, thoracotomy, autopsy, etc.) do not match generally accepted, established, or practiced rules or recommendations?

\begin{tabular}{|c|c|c|}
\hline & Unclear & High \\
\hline \multicolumn{3}{|l|}{ DOMAIN 4: Flow and Timing } \\
\hline $\begin{array}{l}\text { Was there an appropriate interval between index } \\
\text { test and reference standard? }\end{array}$ & Yes & \\
\hline $\begin{array}{l}\text { Did all patients receive the same reference stan- } \\
\text { dard? }\end{array}$ & No & \\
\hline Were all patients included in the analysis? & Yes & \\
\hline $\begin{array}{l}\text { Did all participants receive a reference stan- } \\
\text { dard? (Risk of partial verification bias) }\end{array}$ & Yes & \\
\hline
\end{tabular}

\author{
Abbreviations \\ AP: anteroposterior \\ ATLS: Advanced Trauma Life Support \\ CECT: contrast-enhanced computed tomography \\ CE-MDCT: contrast-enhanced multidetector spiral computed tomography \\ CEUS: contrast-enhanced ultrasound \\ CT: computed tomography
}


CXR: chest radiography

DPL: diagnostic peritoneal lavage

EFAST: extended FAST

ELAP: exploratory laparotomy

ESI: Emergency Severity Index

FAST: focused assessment with sonography in trauma

GCS: Glasgow Coma Scale

ICD-10: 10th revision of the International Statistical Classification of Diseases and Related Health Problems

IQR: interquartile range

ISS: Injury Severity Score

IV: intravenous

MSCT: multislice CT

PTS: Pediatric Trauma Score

WHO: World Health Organization

US: ultrasound/ultrasonography

Characteristics of excluded studies [ordered by study ID]

\begin{tabular}{|c|c|}
\hline Study & Reason for exclusion \\
\hline Abdulrahman 2015 & Insufficient information to calculate diagnostic accuracy \\
\hline Ala 2016 & Inadequate reference standard \\
\hline Arrillaga 1999 & Inadequate reference standard \\
\hline Beck-Razi 2007 & Penetrating trauma \\
\hline Behboodi 2016 & Inadequate diagnostic values \\
\hline Brooks 2004a & Penetrating trauma \\
\hline Brooks 2004b & Inadequate reference standard \\
\hline Brown 2001 & Inadequate reference standard \\
\hline Byars 2013 & Inadequate index test \\
\hline Cook 2015 & Insufficient information to calculate diagnostic accuracy \\
\hline Coskun 2011 & Inadequate reference standard \\
\hline Dan 2010 & Inadequate reference standard \\
\hline Deunk 2010 & Inadequate index test \\
\hline Donmez 2011 & Insufficient information to calculate diagnostic accuracy \\
\hline Faruque 2013 & Inadequate reference standard \\
\hline Hamada 2016 & Insufficient information to calculate diagnostic accuracy \\
\hline Helling 2007 & Insufficient information to calculate diagnostic accuracy \\
\hline Heyn 2008 & Inadequate reference standard \\
\hline Holmes 2012 & Penetrating trauma \\
\hline
\end{tabular}




\begin{tabular}{|c|c|}
\hline Study & Reason for exclusion \\
\hline Hyacinthe 2012 & Penetrating trauma \\
\hline Ianniello 2014 & Insufficient information to calculate diagnostic accuracy \\
\hline Ingeman 1996 & Insufficient information to calculate diagnostic accuracy \\
\hline Jalli 2009 & Inadequate index test \\
\hline Kaya 2015 & Inadequate reference standard \\
\hline Kern 1997 & Penetrating trauma \\
\hline Kirkpatrick 2002 & Inadequate reference standard \\
\hline Kirkpatrick 2004 & Insufficient information to calculate diagnostic accuracy \\
\hline Kirkpatrick 2005 & Inadequate reference standard \\
\hline Krupnick 1997 & Case-control study \\
\hline Ku 2013 & Penetrating trauma \\
\hline Kumar 2014 & Insufficient information to calculate diagnostic accuracy \\
\hline Lichtenstein 2005 & Insufficient information to calculate diagnostic accuracy \\
\hline Matsumoto 2016 & Insufficient information to calculate diagnostic accuracy \\
\hline Mihalik 2012 & Insufficient information to calculate diagnostic accuracy \\
\hline Moylan 2007 & Inadequate reference standard \\
\hline Mumtaz 2016 & Penetrating trauma \\
\hline Nagarsheth 2011 & Penetrating trauma \\
\hline Natarajan 2010 & Insufficient information to calculate diagnostic accuracy \\
\hline Pak-art 2003 & Inadequate reference standard \\
\hline Richards 2004 & Inadequate reference standard \\
\hline Richardson 1997 & Case-report \\
\hline Schleder 2013 & Penetrating trauma \\
\hline Sheng 2013 & Penetrating trauma \\
\hline Smith 2009 & Insufficient information to calculate diagnostic accuracy \\
\hline Smith 2013 & Inadequate reference standard \\
\hline Smith 2015 & Battlefield scenario \\
\hline Soldati 2007 & Insufficient information to calculate diagnostic accuracy \\
\hline
\end{tabular}




\begin{tabular}{|c|c|}
\hline Study & Reason for exclusion \\
\hline Soult 2015 & Penetrating trauma \\
\hline Tajoddini 2013 & Insufficient information to calculate diagnostic accuracy \\
\hline Tam 2005 & PenetratIng trauma \\
\hline Tas 2004 & Insufficient information to calculate diagnostic accuracy \\
\hline Tayal 2006 & Inadequate reference standard \\
\hline Tummers 2016 & Inadequate reference standard \\
\hline Tunuka 2014 & Inadequate reference standard \\
\hline Valentino 2008 & Insufficient information to calculate diagnostic accuracy \\
\hline Van Diepen 2013 & Insufficient information to calculate diagnostic accuracy \\
\hline Vassiliadis 2003 & Penetrating trauma \\
\hline
\end{tabular}

Characteristics of studies awaiting classification [ordered by study ID]

\section{Armstrong 2018}

\section{Study characteristics}

\begin{tabular}{ll}
\hline Patient sampling & Prospective study \\
\hline Patient characteristics and setting & Type of injury: blunt abdominal solid organ injury \\
& $\begin{array}{l}\text { Participants included in analysis: } 18 \\
\text { Age: } 7 \text { to } 18 \text { years } \\
\text { Care setting: level } 2 \text { trauma centre }\end{array}$ \\
\hline Index tests & Index tests: conventional US and CEUS \\
\hline Target condition and reference & Reference standard: CT \\
\hline standard(s) & Time between index test and reference standard: 48 hours \\
\hline Flow and timing & \\
\hline Notes & May be incorporated into the review at the next update \\
\hline
\end{tabular}

\section{Elbaih 2017}

\section{Study characteristics}

Patient sampling Prospective cross-sectional study 


\section{Elbaih 2017 (Continued)}

Patient characteristics and setting

Type of injury: polytraumatised patients with a blunt mechanism

Participants included in analysis: 150

Age: mean age $27.98 \pm 20.39$ years

Haemodynamic stability: unstable

Index tests
$\begin{aligned} & \text { Target condition and reference stan- } \\ & \text { dard(s) }\end{aligned}$

Flow and timing

Comparative

Notes May be incorporated into the review at the next update

Hsu 2017

\section{Study characteristics}

\begin{tabular}{|c|c|}
\hline Patient sampling & Retrospective study \\
\hline \multirow{3}{*}{$\begin{array}{l}\text { Patient characteristics and } \\
\text { setting }\end{array}$} & Type of injury: blunt abdominal trauma \\
\hline & Participants included in analysis: 438 \\
\hline & $\begin{array}{l}\text { Inclusion criteria: patients who had a FAST examination performed by qualified residents and had } \\
\text { received subsequent formal radiographic or surgical evaluations }\end{array}$ \\
\hline Index tests & Index test: FAST US \\
\hline $\begin{array}{l}\text { Target condition and refer- } \\
\text { ence standard(s) }\end{array}$ & $\begin{array}{l}\text { Reference standard: subsequent surgical findings or formal Department of Radiology reference } \\
\text { standards }\end{array}$ \\
\hline \multicolumn{2}{|l|}{ Flow and timing } \\
\hline \multicolumn{2}{|l|}{ Comparative } \\
\hline Notes & May be incorporated into the review at the next update \\
\hline
\end{tabular}

\section{Kozaci 2018}

\section{Study characteristics}

\begin{tabular}{ll}
\hline Patient sampling & Prospective study \\
\hline Patient characteristics and setting & Type of injury: multiple trauma with thoracic injuries \\
& Participants included in analysis: 81 \\
Age: $>18$ years \\
\hline
\end{tabular}


Kozaci 2018 (Continued)

Index tests

Index test: bedside thoracic US

Target condition and reference standard(s)

Reference standard: thoracic CT

Flow and timing

Comparative

Notes May be incorporated into the review at the next update

Maximus 2018

\section{Study characteristics}

\begin{tabular}{|c|c|}
\hline Patient sampling & Retrospective study \\
\hline \multirow[t]{3}{*}{ Patient characteristics and setting } & Type of injury: pneumothorax \\
\hline & Participants included in analysis: 300 \\
\hline & Care setting: level 1 urban trauma centre \\
\hline Index tests & Index tests: EFAST and chest X-ray \\
\hline Target condition and reference standard(s) & Reference standard: CT \\
\hline \multicolumn{2}{|l|}{ Flow and timing } \\
\hline \multicolumn{2}{|l|}{ Comparative } \\
\hline Notes & May be incorporated into the review at the next update \\
\hline
\end{tabular}

Mumtaz 2017

\section{Study characteristics}

\begin{tabular}{ll}
\hline Patient sampling & Prospective study \\
\hline Patient characteristics and setting & Type of injury: blunt or penetrating trauma \\
& Participants included in analysis: 80 \\
& Haemodynamic stability: stable \\
& $\begin{array}{l}\text { Age: } 57 \text { males with mean age } 27.30 \pm 9.69 \text { years and } 23 \text { females with mean age } 30.91 \pm 11.58 \\
\text { years }\end{array}$ \\
\hline Index tests & Index tests: Portable bed side US and supine chest radiograph \\
\hline $\begin{array}{l}\text { Target condition and reference } \\
\text { standard(s) }\end{array}$ & Reference standard: CT \\
\hline
\end{tabular}

\section{Flow and timing}


Mumtaz 2017 (Continued)

Comparative

Notes May be incorporated into the review at the next update

\section{Sauter 2017}

\section{Study characteristics}

\begin{tabular}{ll}
\hline Patient sampling & Retrospective cross-sectional study \\
\hline Patient characteristics and setting & Type of injury: multiple blunt trauma with pneumothorax \\
& Participants included in analysis: 106 \\
& Care Setting: level 1 trauma centre \\
& Age: mean age 48.6 \pm 19.3 years \\
\hline Index tests & Index tests: EFAST \\
\hline $\begin{array}{l}\text { Target condition and reference stan- } \\
\text { dard(s) }\end{array}$ & Reference standard: CT \\
\hline Flow and timing & \\
\hline Comparative & May be incorporated into the review at the next update \\
\hline Notes &
\end{tabular}

Waheed 2018

\section{Study characteristics}

\begin{tabular}{ll}
\hline Patient sampling & Retrospective study \\
\hline Patient characteristics and setting & Type of injury: blunt abdominal injury caused by motor vehicle accident \\
& Participants included in analysis: 105 \\
& Age: mean age $32.3 \pm 19.3$ years (range 15 to 56 years) \\
& Haemodynamic stability: stable \\
\hline Index tests & Index tests: FAST \\
\hline
\end{tabular}

Target condition and reference stan$\operatorname{dard}(\mathrm{s})$

\section{Reference standard: CT}

\section{Flow and timing}

\section{Comparative}
Notes
May be incorporated into the review at the next update 
Zieleskiewicz 2018

\section{Study characteristics}

Patient sampling Retrospective study

Patient characteristics and setting
Type of injury: severe trauma

Participants included in analysis: 756

Care Setting: level 1 trauma centre

Index tests Index tests: EFAST, chest X-ray, pelvic X-ray

Target condition and reference standard(s) Reference standard: total body CT

Flow and timing

Comparative

Notes May be incorporated into the review at the next update

\section{Abbreviations}

CEUS: contrast-enhanced ultrasound

CT: computed tomography

EFAST: extended FAST

FAST: focused assessment with sonography in trauma

US: ultrasound

\section{A T A}

Presented below are all the data for all of the tests entered into the review.

\section{Table Tests. Data tables by test}

\begin{tabular}{ll}
\hline Test & No. of studies \\
\hline 1 Main analysis set & 34 \\
\hline $\begin{array}{l}\text { 2 Sensitivity analysis set with lower sensitivity/specificity values in two original } \\
\text { studies }\end{array}$ & 34 \\
\hline
\end{tabular}

Test 1. Main analysis set.

Test 2. Sensitivity analysis set with lower sensitivity/specificity values in two original studies. 


\section{A P P E N D I C E S}

\section{Appendix 1. Search strategies}

\section{Ovid MEDLINE Databases}

Epub Ahead of Print, In-Process \& Other Non-Indexed Citations, Ovid MEDLINE(R) Daily and Ovid $\operatorname{MEDLINE}(\mathrm{R})<1946$ to Present $>$

The MEDLINE search strategy is based on the following structure:

Search1. Target Condition + Index Test (POC Ultrasonography)

OR

Search 2. Target Condition + Index Test (Ultrasonography) + Reference Standard + DTA Filter + Setting)

[Target Condition]

1 exp Abdominal Injuries/

2 exp Thoracic Injuries/

3 ((chest or torso) adj3 (injur* or trauma*)).ti,ab,kf.

4 ((injur ${ }^{\star}$ or ruptur ${ }^{\star}$ or bleed ${ }^{\star}$ or trauma*) adj3 (abdom* ${ }^{\star}$ or thorax or thoracic or thoracoabdom ${ }^{\star}$ or thoraco-abdom ${ }^{\star}$ or stomach or gastric $\left.\left.{ }^{\star}\right)\right) . t i, a b, k f$.

5 (free fluid adj3 (abdom* or thorax or thoracic or thoracoabdom* or thoraco-abdom)).ti,ab,kf.

6 pneumothorax/

7 hemopneumothorax/

8 hemothorax/

9 (pneumothor ${ }^{\star}$ or h?emopneumothor* or h?emothor ${ }^{\star}$ ).ti,ab,kf.

10 (retroperitoneal or retro-peritoneal or intraperitoneal or intra-peritoneal or mediastinum or

pericardium).ti,ab,kf,hw.

11 *"Wounds and Injuries"/

12 "Wounds and Injuries"/dg [Diagnostic Imaging]

13 Wounds, Nonpenetrating/

14 rupture/ or exp splenic rupture/ or stomach rupture/

15 ((injur ${ }^{\star}$ or ruptur* ${ }^{\star}$ or bleed* or trauma* or lacerat* or tear? or contusion*) adj3 (spleen* or splenic or hepatic or visceral or liver* or

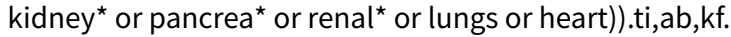

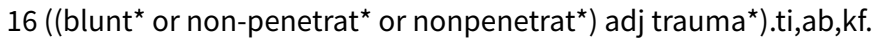

17 (polytrauma* or poly trauma* or multiple trauma* or mass casualt ${ }^{\star}$ ).ti,ab,kf,hw.

18 or/1-17

[Index Test]

19 exp Ultrasonography/

20 Diagnostic Imaging/

21 (ultraso* or sonogra*).ti,ab,kf.

22 (diagnos $^{\star}$ adj2 (screen* or scan* or imag*)).ti,ab,kf.

23 diagnostic imaging.fs.

24 (advanced trauma life support or atls).ti,ab,kf.

25 or/19-24

26 point of care.ti,ab,kf.

27 Point-of-Care Systems/

28 (POCUS or POC US or POC USG).ti,ab,kf.

29 ((focused adj2 assessment adj2 sonogra* adj2 trauma) or extended-FAST or (FAST adj (ultrasonography or ultrasound))).ti,ab,kf.

30 ((portable or hand-held or handheld or mobile or emergency) adj (sonogra* or ultraso*)).ti,ab,kf.

31 bedside.ti,ab,kf.

32 or/26-31

[Search1: Target Condition + Index Test (POC Ultrasonography)]

33 (18 and 25 and 32)

[Reference Standard]

34 exp Tomography, X-Ray Computed/

35 (CT scan or cat scan or (xray* adj1 ct) or xrayct or (compute* adj2 tomograph*)).ti,ab,kf.

36 (MDCT or pan scan or panscan).ti,ab,kf.

37 (laparotom* or laparoscop ${ }^{\star}$ or thoracotom ${ }^{\star}$ or sternotom ${ }^{\star}$ or thoracoscop* or autops $\left.{ }^{\star}\right) . t i, a b, k f, h w$.

38 or/34-37

[DTA Filter]

39 "sensitivity and specificity"/ or "limit of detection"/ or roc curve/ or signal-to-noise ratio/ or "predictive value of tests"/

40 "reproducibility of results"/ 
41 likelihood ratio*.ti,ab,kf.

42 ((re-test or retest or test-retest or test-re-test) adj reliability).ti,ab,kf.

43 receiver operating characteristic ${ }^{\star} . \mathrm{ti}, \mathrm{ab}, \mathrm{kf}$.

44 (ROC adj5 (analy* or curve or curves)).ti,ab,kf.

45 or/39-44

[Search2: Target Condition + Index Test (Ultrasonography/Diagnostic Imaging (MeSH)) + Reference Standard + DTA Filter]

4618 and ( 19 or 21 ) and 38 and 45

[Limited to Setting]

47 (trauma* or emergenc* or bedside?).ti,ab,kf,hw.

48 (46 and 47)

[Search 1 or Search 2]

49 (33 or 48)

50 remove duplicates from 49

\section{Ovid Embase $<\mathbf{1 9 7 4}$ to date $>$}

As Embase records are more highly indexed (compared to MEDLINE and other database records), there will be one search strand, based on the following structure: Target Condition + Index Test (POC Ultrasonography) + (Setting OR Reference Standard OR DTA Filter)

\section{[Target Condition]}

1 exp *abdominal injury/ or exp abdominal injury/di

$2 \exp { }^{*}$ thorax injury/ or exp *thorax injury/di

3 ((chest or torso) adj3 (injur ${ }^{\star}$ or trauma*)).ti,ab,kw.

4 ((injur ${ }^{\star}$ or ruptur ${ }^{\star}$ or bleed ${ }^{\star}$ or trauma*) adj3 (abdom* or thorax or thoracic or thoracoabdom ${ }^{\star}$ or thoraco-abdom ${ }^{\star}$ or stomach or gastric $\left.\left.{ }^{\star}\right)\right) . t i, a b, k w$.

5 (free fluid and (abdom* or thorax or thoracic or thoracoabdom* or thoraco-abdom*)).ti,ab,kw.

6 pneumothorax/ or hematopneumothorax/ or spontaneous pneumothorax/ or tension pneumothorax/

7 hematothorax/

8 (pneumothor ${ }^{\star}$ or h?emopneumothor ${ }^{\star}$ or h?emothor ${ }^{\star}$ ).ti,ab,kw.

9 ((fluid ${ }^{\star} 1$ or blood or bleed $\left.{ }^{\star}\right)$ and (retroperitoneal or retro-peritoneal or intraperitoneal or intra-peritoneal or

mediastinum or pericardium)).ti,ab,kw, hw.

10 blunt trauma/ or crush trauma/

11 * rupture/ or exp *digestive system rupture/ or *spleen rupture/ or exp *thorax organ rupture/

12 rupture/di or exp digestive system rupture/di or spleen rupture/di or exp thorax organ rupture/di

13 ((injur* or ruptur ${ }^{\star}$ or bleed ${ }^{\star}$ or trauma* or lacerat* or tear? or contusion*) adj3 (spleen* or splenic or hepatic or visceral or liver ${ }^{\star}$ or

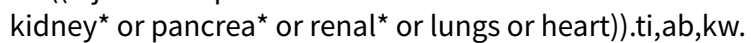

14 ((blunt* or non-penetrat* or nonpenetrat*) adj trauma*).ti,ab,kw.

15 (polytrauma* or poly trauma* or (multiple adj2 trauma*) or mass casualt*).ti,ab,kw,hw.

16 or/1-15

[Index Test]

17 exp echography/

18 diagnostic imaging/

19 (ultraso* or sonogra*).ti,ab,kw.

20 (diagnos $^{\star}$ adj2 (screen* or scan* or imag*)).ti,ab,kw.

21 (advanced trauma life support or atls).ti,ab,kw.

22 or/17-21

23 point of care.ti,ab,kw.

24 "point of care testing"/

25 (POCUS or POC US or POC USG).ti,ab,kw.

26 ((focused adj2 assessment adj2 sonogra* adj2 trauma) or extended-FAST or (FAST adj (ultrasonography or ultrasound))).ti,ab,kw.

27 ((portable or hand-held or handheld or mobile or emergency) adj (sonogra* or ultraso*)).ti,ab,kw.

28 bedside?.ti,ab,kw.

29 or $/ 23-28$

[Target Condition + Index Test (POC Ultrasonography)]

30 (16 and 22 and 29)

[Setting]

31 (trauma* or emergenc $^{\star}$ or ((acute or critical or intensive) adj2 (care or medicine))).ti,ab,kw,hw.

[Reference Standard]

32 exp computer assisted tomography/

33 (CT scan or cat scan or (xray* adj1 ct) or xrayct or (compute* adj2 tomograph*)).ti,ab,kw.

34 (MDCT or pan scan or panscan).ti,ab,kw. 
35 thorax radiography/

36 (laparotom* or laparoscop* or thoracotom ${ }^{\star}$ or sternotom ${ }^{\star}$ or thoracoscop* or autops*).ti,ab,kw,hw.

37 or/32-36

[DTA Filter]

38 diagnostic accuracy/

39 "sensitivity and specificity"/

40 receiver operating characteristic/

41 predictive value/

42 intermethod comparison/ or comparative study/

43 ((re-test or retest or test-retest or test-re-test) adj reliability).ti,ab,kw.

44 likelihood ratio*.ti,ab,kw.

45 receiver operating characteristic*.ti,ab,kw.

46 (ROC adj5 (analy* or curve or curves)).ti,ab,kw.

47 (detect $^{\star}$ or diagnos $\left.{ }^{\star}\right) . \mathrm{ti}, \mathrm{ab}, \mathrm{kw}$.

48 or $/ 38-47$

[Target Condition + Index Test (POC Ultrasonography) + (Setting OR Reference Standard OR DTA Filter)]

49 ( 30 and ( 31 or 37 or 48$)$ )

50 Animal experiment/ not (human experiment/ or human/)

51 (exp animal/ or nonhuman/) not ((exp animal/ or nonhuman/) and (human/ or human experiment/))

52 (rat or rats or mouse or mice or swine or porcine or murine or sheep or lambs or pigs or piglets or rabbit or

rabbits or cat or cats or feline or dog or dogs or canine or cattle or bovine or monkey or monkeys or trout or

marmoset $\left.{ }^{\star}\right)$.ti.

53 case report/

54 or $/ 50-53$

55 (49 not 54)

56 limit 55 to (article in press status or conference abstract status or embase status or inprocess status)

57 remove duplicates from 56

\section{PubMed (NOT MEDLINE) <1947 to date>}

\#18 (\#1 AND \#17)

\#17 (\#5 AND \#16)

\#16 (\#6 OR \#7 OR \#8 OR \#9 OR \#10 OR \#11 OR \#12 OR \#13 OR \#14 OR \#15)

\#15 ("free fluid" OR "free fluids")

\#14 ((pneumothorax OR pneumothoracic OR pneumothoraces OR hemopneumothorax OR hemopneumothoracic OR hemopneumothoraces OR haemopneumothorax OR haemopneumothoracic OR haemopneumothoraces OR hemothorax OR hemothoracic OR hemothoraces OR haemothorax OR haemothoracic OR haemothoraces))

\#13 ("Pneumothorax"[Mesh] OR "Hemopneumothorax"[Mesh])

\#12 ((polytrauma OR “poly trauma” OR “multiple trauma” OR “multiple traumas” OR “mass casualties” OR “mass casualty”))

\#11 "Mass Casualty Incidents"[Mesh]

\#10 ((blunt OR non-penetrating OR nonpenetrating) AND (trauma OR traumatic))

\#9 ((retroperitoneal OR retro-peritoneal OR intraperitoneal OR intra-peritoneal OR mediastinum OR pericardium))

\#8 ((spleen OR splenic OR hepatic OR liver OR renal OR kidney OR kidneys OR pancreas OR pancreatic OR lung OR lungs OR heart OR visceral) AND (injury OR injuries OR trauma OR traumatic OR rupture OR ruptured OR bleed OR bleeding OR lacerate OR laceration OR tear OR teared OR contusion OR wound OR wounds))

\#7 ((abdomen OR abdominal OR chest OR thorax OR thoracic OR torso OR thoracoabdomen OR thoracoabdominal OR thoraco-abdomen OR thoraco-abdominal OR stomach OR gastric) AND (injury OR injuries OR trauma OR traumatic OR rupture OR ruptured OR bleed OR bleeding OR wound OR wounds))

\#6 (“Abdominal Injuries"[Mesh] OR "Thoracic Injuries"[Mesh] OR "Wounds, Nonpenetrating"[Mesh])

\#5 (\#2 OR \#3 OR \#4)

\#4 (POCUS OR POC-US OR POC-USG OR "POC US" OR "POC USG")

\#3 (( (point-of-care OR "point of care" OR "focused assessment" OR "focussed assessment" OR EFAST OR bedside OR bedsides OR portable OR hand-held OR handheld OR mobile OR emergency) AND (sonograph OR sonography OR sonographic OR sonographer OR sonographers OR ultrasonography OR ultrasonographic OR ultrasound)))

\#2 "Point-of-Care Systems"[Mesh]

\#1 (pubmednotmedline[sb] OR publisher[sb] OR in process[sb])

BIOSIS Previews (Web of Science) $<1926$ to date $>$ 
Topic $=\left(\right.$ thorax injur ${ }^{\star}$ OR thoracic injur ${ }^{\star}$ OR chest injur* OR torso injur ${ }^{\star}$ OR thoracoabdominal injur ${ }^{\star}$ OR thoraco-abdominal injur ${ }^{\star}$ OR abdominal injur* OR abdomen injur ${ }^{\star}$ thorax injur ${ }^{\star}$ OR stomach injur ${ }^{\star}$ OR gastric injur* OR thoracic trauma OR chest trauma OR torso trauma OR thoracoabdominal trauma OR thoraco-abdominal trauma OR abdominal trauma OR abdomen trauma OR stomach trauma OR gastric trauma OR pneumothorax OR hemopneumothorax OR haemopneumothorax OR hemothorax OR haemothorax OR pneumothor ${ }^{\star}$ OR retroperitoneal OR retro-peritoneal OR intraperitoneal OR intra-peritoneal OR mediastinum OR spleen trauma OR spleen injur ${ }^{\star}$ OR splenic trauma OR splenic injur ${ }^{\star}$ OR hepatic trauma OR hepatic injur ${ }^{\star}$ OR visceral trauma OR visceral injur ${ }^{\star}$ OR liver trauma OR liver injur ${ }^{\star}$ OR kidney trauma OR kidney injur* OR pancrea* trauma OR pancrea* injur* OR renal trauma OR renal injur* OR lung trauma OR lung injur* OR free fluid OR free air OR organ injur* OR organ lesion* OR vascular lesion* OR blunt trauma OR polytrauma* OR multiple trauma*) AND Topic $=$ (ultraso* OR US OR sonogra* OR diagnos* screen* OR diagnos* scan $^{\star}$ OR diagnos* imag* OR point of care OR point-of-care OR POCS OR POCUS OR POC US OR POC USG OR FAST OR focused assessment OR EFAST OR extended-FAST OR ALTS OR advanced trauma life support)

\section{Appendix 2. Review-specific QUADAS-2 coding manual}

\section{Domain 1: Participant selection}

\begin{tabular}{ll}
\hline Risk of bias & $\begin{array}{l}\text { Q1. Was a consecutive or ran- } \\
\text { dom sample of participants } \\
\text { enrolled? }\end{array}$ \\
& - ' 'No' if a non-random (or non-consecutive) selection method was used \\
& - 'Unclear' if the procedures are only partially reported and you feel that \\
both 'yes' or 'no' are inadequate
\end{tabular}

Q2. Did the study avoid inappropriate exclusions (e.g. exclusion of patients with underlying diseases associated with intra-abdominal fluid)

Summary judgement of risk of bias
- 'Yes' if there were no inappropriate exclusions

- 'No' if there were inappropriate exclusions

- 'Unclear' if it is not clearly stated if there are any inappropriate exclusions

- Low risk: both answers are 'yes'

- High risk: $\geq 1$ answers are 'no'

- Unclear risk: all other cases

Concerns regarding ap- Q3. Are there concerns that plicability the included patients and setting do not match the review question?
- 'Low' if all patients with blunt thoracoabdominal and/or multiple trauma are the unit of investigation, and if the population characteristics are representative of those who will receive the test in practice

- 'High' if patients with any type of acute abdomen are enrolled, or when there are other covariates that are reason for concern

- 'Unclear' if answering 'low' or 'high' is inappropriate

\section{Domain 2: Index test}

Risk of bias Q1. Were the index test results interpreted without knowledge of the results of the reference standard?

\section{- 'Yes' if there is a statement that the index test results were interpreted blind to the results of the reference test}

- 'No' if this does not appear to be the case

- 'Unclear' if this information was not reported
- 'Yes' if the threshold values were prespecified before the start of the study

- 'No' if the threshold values were selected on the basis of the collected data

- 'Unclear' if there is insufficient information to make a judgement 
Q3. Was the qualification of the ultrasound operator appropriate?

- 'Yes' if his/her background, training, and experience were sufficient according to clinical expert rating (e.g. the operator was a board-certified/qualified trauma or emergency surgeon, radiologist, sonographer, etc.)

- 'No' if the qualification was rated as not appropriate by clinical experts

- 'Unclear' if this information was not reported

Q4. Was the ultrasound hardware (i.e. generation, manufacturer, probe, etc.) up-todate?

- 'Yes' if the technical features were up-to-date according to clinical expert rating (e.g. by mentioning the ultrasound hardware and probes (frequency) used)

- 'No' if the ultrasound hardware was rated as outdated

- 'Unclear' if this information was not reported

Q5. Was the ultrasound protocol (i.e. 'classic' focused assessment with sonography in trauma (FAST)) appropriate?

- 'Yes' if the ultrasound protocol (e.g. screening for free fluid and/or air, organ lacerations, etc.) was appropriate according to clinical expert rating

- 'No' if this was not the case

- 'Unclear' if this information was not reported

Summary judgement of risk - Low risk: $\geq 4$ answers are 'yes'

of bias

- High risk: $\geq 3$ answers are 'no'

- Unclear risk: all other cases

Concerns regarding applicability
Q6: Are there concerns that the definition or performance of the index test (i.e. point-ofcare ultrasonography (POCS) for trauma) do not match generally accepted, established, or practiced rules or recommendations?
- 'Low concern' all patients with blunt thoracoabdominal and/or multiple trauma are screened with POCS in an established or recommended manner

- 'High concern' ultrasound protocols deviate significantly from established protocols; patients with any type of acute abdomen were enrolled; or other covariates were a reason for concern

- 'Unclear concern' if answering 'low' or 'high' is inappropriate

Domain 3: Reference test

\section{Risk of bias}

Q1. Is this the type of test that is likely to correctly classify the target condition?

- 'Yes' when computed tomography (CT) and/or magnetic resonance imaging (MRI), or any invasive procedure like laparotomy/laparoscopy, thoracotomy/thoracoscopy, or autopsy was used as a reference standard

- 'No' if no reference test was specified, or only positive POCS findings were confirmed by an imaging and/or invasive reference test

- 'Unclear' if answering 'yes' or 'no' is inappropriate

Q2. Were the reference standard results interpreted without knowledge of the results of the index tests?
- 'Yes' if there is a statement that the reference standard results were interpreted blind to the results of the index test

- 'No' if this does not appear to be the case

- 'Unclear' if this information was not reported

Q3. Was the qualification of doctors (i.e. radiologists, surgeons, etc.) determining the
- 'Yes' if his/her background, training, and experience were sufficient according to clinical expert rating (e.g. the operator was a board-certi- 
reference standard appropri- fied/qualified trauma or emergency surgeon, radiologist, sonographer, ate? etc.)

- 'No' if the qualification was rated as not appropriate by clinical experts

- 'Unclear' if this information was not reported

Q4. Was the reference imaging standard (i.e. multidetector-row computed tomography (MDCT) rows ( 4 to $\geq$ 256 slices), contrast imaging, etc.) up-to-date?
- 'Yes' if the technical features were up-to-date according to clinical expert rating (e.g. by mentioning the hardware and imaging protocols used)

- 'No' if the CT hardware or imaging protocols were rated as outdated

- 'Unclear' if this information was not reported

Summary judgement of risk - Low risk: $\geq 3$ answers are 'yes'

of bias

- High risk: $\geq 2$ answers are 'no'

- Unclear risk: all other cases

Concerns regarding applicability
Q5: Are there concerns the definition or performance of the reference tests (e.g. $\mathrm{CT}, \mathrm{MRI}$, laparotomy, thoracotomy, autopsy, etc.) do not match generally accepted, established, or practiced rules or recommendations?
- 'Low concern' all patients with blunt thoracoabdominal or multiple trauma, or both undergo a thoracoabdominal or whole-body CT scan irrespective of clinical or ultrasound findings

- 'High concern' reference tests are ordered selectively or conditional on clinical or ultrasound results

- 'Unclear concern' if answering 'low' or 'high' concern is inappropriate

\section{Domain 4: Flow and timing}

\section{Risk of bias}

Q1. Was there an appropriate interval between index test and reference standard? ceive a reference standard? (Risk of partial verification bias)

- 'Yes' if the POCS scan was followed by any reference test within 30 minutes

- 'No' if the reference test was executed after 30 minutes, if the reference test was employed before POCS, or in case of any other conditions

- 'Unclear' if this information was not reported

- 'Yes' if it is clear that all (or a random selection of) participants who received the index test also received the reference test

- 'No' if participants did or did not receive a reference test based on the outcome of the index test, or the selection of participants to receive the reference test was not random

- 'Unclear' if this information was not reported

Q3. Did all participants receive the same (or any equivalent) reference standard?

- 'Yes' if all participants had the same (or an equivalent) reference standard

- 'No' if this was not the case

- 'Unclear' if this information was not reported

Q4. Were all participants included in the analysis?
- 'Yes' if all participants entered into the study were included in the analysis

- 'No' if it appears that some of the participants were excluded from the analysis for whatever reason (e.g. did not complete the study, dubious test results) 
Summary judgement of risk - Low risk: $\geq 3$ answers are 'yes'

of bias

- High risk: $\geq 2$ answers are 'no'

- Unclear risk: all other cases

\section{CONTRIBUTIONS OF AUTHORS}

DS conceived the review and wrote the first draft of the protocol.

$\mathrm{JL}$ provided clinical guidance and contributed to the revision of the protocol.

AH provided statistical guidance and contributed to the revision of the protocol.

$\mathrm{AH}$ and JL screened the search results, selected studies, and extracted data.

AH performed the statistical analysis.

AE, SM, and PF provided distinct clinical expertise.

PF also evaluated this review with regard to its relevance for the USA healthcare system.

All authors contributed to the writing of the review, which was supervised by DS.

\section{DECLARATIONSOF INTEREST}

D Stengel: none known

J Leisterer: none known

P Ferrada: none known

A Ekkernkamp: none known

S Mutze: none known

A Hoenning: none known

\section{SOURCES OF SUPPORT}

\section{Internal sources}

- Centre for Clinical Research, Department of Trauma and Orthopaedic Surgery, Unfallkrankenhaus Berlin, Berlin, Germany.

\section{External sources}

- National Institute for Health Research (NIHR), UK.

This project is supported by the UK NIHR, through Cochrane Infrastructure funding to the Cochrane Injuries Group. The views and opinions expressed are those of the authors and do not necessarily reflect those of the Systematic Reviews Programme, NIHR, National Health Service (NHS), or the Department of Health.

\section{DIFFERENCES BETWEEN PROTOCOLANDREVIEW}

In Investigations of heterogeneity, we modified the categorisation of two potential sources of heterogeneity (i.e. reference standard and participant age) since the original classification did not result in sufficiently large subgroups. We were not able to compare single reference standards because each study used computed tomography (CT) as the diagnostic reference instrument, either alone or in combination with additional tests. With respect to participant age, we only compared studies that included patients under 18 years of age versus studies that included adults and mixed-age populations, due to the lack of adult-only studies. We made this decision before analysing the data. We could not explore various secondary sources of heterogeneity (i.e. environment, operator's expertise and background, hardware, test thresholds) due to missing information. 
We intended to conduct Sensitivity analyses to investigate the influence of study quality based on the risk-of-bias assessment. We performed two additional analyses which were not prespecified in the protocol: evaluating the effect of two independent reviewers and diagnostic accuracy in a children-only cohort.

In the Assessment of methodological quality section, we removed one redundant item relating to case-control studies from the QUADAS-2 tool because these studies were already omitted from the review according to our predefined exclusion criteria.

We did not contact authors of individual studies as originally planned, since we required no further information or raw data.

The clinical experts Axel Ekkernkamp, Sven Mutze, and Paula Ferrada contributed to the full review and were added as authors.

\section{INDEX TERMS}

\section{Medical Subject Headings (MeSH)}

*Point-of-Care Systems; Abdominal Injuries [ ${ }^{\star}$ diagnostic imaging]; Age Factors; Focused Assessment with Sonography for Trauma [ ${ }^{*}$ methods]; Reference Standards; Sensitivity and Specificity; Thoracic Injuries [ ${ }^{*}$ diagnostic imaging]; Wounds, Nonpenetrating [ ${ }^{\star}$ diagnostic imaging]

\section{MeSH check words}

Adult; Child; Female; Humans; Male 